\title{
The cosmological Higgstory of the vacuum instability
}

\author{
José R. Espinosa, ${ }^{a, b}$ Gian F. Giudice, ${ }^{c}$ Enrico Morgante, ${ }^{d}$ Antonio Riotto, ${ }^{d}$ \\ Leonardo Senatore, ${ }^{e}$ Alessandro Strumia ${ }^{f, g}$ and Nikolaos Tetradis $^{h}$ \\ ${ }^{a}$ IFAE, Universitat Autónoma de Barcelona, \\ 08193 Bellaterra, Barcelona, Spain \\ ${ }^{b}$ ICREA, Institució Catalana de Recerca i Estudis Avançats, \\ Barcelona, Spain \\ ${ }^{c}$ CERN, Theory Division, \\ CH-1211 Geneva 23, Switzerland \\ ${ }^{d}$ Département de Physique Théorique and Centre for Astroparticle Physics (CAP), \\ Université de Genève, 24 quai Ernest-Ansermet, CH-1211 Geneva 4, Switzerland \\ e Stanford Institute for Theoretical Physics \\ and Kavli Institute for Particle Astrophysics and Cosmology, \\ Physics Department and SLAC, Stanford, CA 94025, U.S.A. \\ ${ }^{f}$ Dipartimento di Fisica dell'Università di Pisa and INFN, Polo Fibonacci, \\ Largo B. Pontecorvo 3, Pisa, Italy \\ ${ }^{g}$ National Institute of Chemical Physics and Biophysics, \\ Ravala 10, Tallinn, Estonia \\ ${ }^{h}$ Department of Physics, University of Athens, \\ Zographou 157 84, Greece \\ E-mail: jose.espinosa@cern.ch, Gian.Giudice@cern.ch, \\ enrico.morgante@unige.ch, antonio.riotto@unige.ch, \\ senatore@stanford.edu, astrumia@cern.ch, ntetrad@phys.uoa.gr
}

Abstract: The Standard Model Higgs potential becomes unstable at large field values. After clarifying the issue of gauge dependence of the effective potential, we study the cosmological evolution of the Higgs field in presence of this instability throughout inflation, reheating and the present epoch. We conclude that anti-de Sitter patches in which the Higgs field lies at its true vacuum are lethal for our universe. From this result, we derive upper bounds on the Hubble constant during inflation, which depend on the reheating temperature and on the Higgs coupling to the scalar curvature or to the inflaton. Finally we study how a speculative link between Higgs meta-stability and consistence of quantum gravity leads to a sharp prediction for the Higgs and top masses, which is consistent with measured values.

KeYwords: Higgs Physics, Standard Model

ARXiv EPRINT: 1505.04825 


\section{Contents}

1 Introduction 1

2 Gauge dependence of the SM effective potential 4

2.1 Gauge (in)dependence of the effective action 5

2.2 Effective potential including only log-enhanced corrections 8

3 Higgs fluctuations during inflation $\quad 10$

$\begin{array}{ll}3.1 \text { Higgs fluctuations during inflation for } \xi_{H}=0 & 10\end{array}$

$\begin{array}{lll}3.2 & \text { Higgs fluctuations during inflation for } \xi_{H} \neq 0 & 14\end{array}$

$\begin{array}{lll}3.3 & \text { Bubble evolution in de Sitter spacetime } & 17\end{array}$

4 Higgs evolution after inflation $\quad 21$

4.1 Higgs evolution during pre-heating 22

$\begin{array}{lll}4.2 & \text { Higgs evolution during reheating } & 23\end{array}$

$\begin{array}{lll}4.3 & \text { Bubble evolution in Minkowski spacetime } & 27\end{array}$

4.4 The Higgs potential for $\xi_{H} \neq 0$ at zero temperature 30

$5 \quad$ The quantum gravity prediction for the Higgs mass 31

6 Conclusions $\quad 35$

$\begin{array}{ll}\text { A Evolution of bubbles } & \mathbf{3 7}\end{array}$

$\begin{array}{lll}\text { A.1 Matching the geometries } & 38\end{array}$

$\begin{array}{lll}\text { A.2 } & \text { Bubbles in asymptotically flat spacetime } & 39\end{array}$

$\begin{array}{lll}\text { A.3 Evolution of positive-mass bubbles } & 42\end{array}$

A.4 Evolution of negative-mass bubbles $\quad 45$

A.5 The AdS 'crunch' 46

A.6 Critical bubbles 48

$\begin{array}{lll}\text { A.7 } & \text { Bubbles in asymptotically de Sitter spacetime } & 49\end{array}$

\section{Introduction}

The measurements of the Higgs-boson and top-quark masses imply the surprising fact that, in the context of the Standard Model (SM) with no additional physics, our universe lies at the edge between stability and instability of the electroweak vacuum [1-3] (see [4-13] for earlier analyses). For the present best fit values of the SM parameters, the Higgs potential develops an instability well below the Planck scale, but the proximity to the stability region insures that the electroweak vacuum lifetime can be exceedingly longer than the age of the universe. 
This intriguing result offers a testing ground for phenomena occurring in the early universe. Indeed, the presence of a minimum of the SM potential deeper than the electroweak vacuum raises many cosmological issues: how did the Higgs field end up today in the false vacuum? Why didn't the primordial dynamics destabilise the Higgs field? How did patches of the universe with large Higgs values evolve in time without swallowing all space? Addressing these questions leads to interesting constraints on early-time phenomena and inflationary dynamics. These constraints are the subject of this paper. Several aspects about electroweak-vacuum decay from thermal or inflationary Higgs fluctuations have already been studied in the literature [14-24], but here we give a comprehensive description of the phenomenon and reach new conclusions.

The effects of the thermal bath during the radiation-dominated phase of the universe are twofold. On one side, thermal fluctuations can trigger nucleation of bubbles that probe Higgs-field values beyond the instability barrier. On the other side, thermal corrections to the Higgs potential tend to stabilise low field values, creating an effective barrier. For the observed values of the SM parameters, the latter effect is dominant and thermal corrections do not destabilise the electroweak vacuum, even when the reheating temperature is close to the Planck scale [14].

More subtle is the issue of the Higgs-field fluctuations generated during inflation. In the case in which the Higgs has no direct coupling to the inflaton and is minimally coupled to gravity (and hence is effectively massless during inflation), the field develops fluctuations with amplitude proportional to $H$, the Hubble rate during inflation. These fluctuations pose a threat to vacuum stability. For values of $H$ smaller than the height of the potential barrier, the Higgs field can tunnel into anti-de Sitter (AdS), according to the Coleman-de Luccia bubble nucleation process [25]. When $H$ becomes comparable to the barrier height, the transition is well described by the Hawking-Moss instanton [26], which corresponds to a thermal overcoming of the barrier due to the effective Gibbons-Hawking temperature $T=H / 2 \pi[27]$ associated with the causal horizon of de Sitter (dS) space. However, a more convenient way to compute the evolution of the Higgs fluctuations during inflation is through a stochastic approach based on a Fokker-Planck equation that describes the probability to find the Higgs field at a given value $h$ and time $t$ [28-31]. This approach was followed in $[14,23,24]$ to derive the probability distribution of Higgs patches in the universe. In this paper, we describe the long-wavelength modes of the Higgs field using a Langevin equation sourced by a Gaussian random noise that mimics quantum fluctuations during inflation. This method has the advantage of bypassing the problem of choosing boundary conditions and it is shown to agree with the results from the Fokker-Planck approach with appropriate boundary conditions.

Quantifying the probability for the existence of a patch of the Higgs field in the SM vacuum sufficiently large to encompass our observable universe is a subtle issue, which requires an understanding of how AdS bubbles (with large Higgs-field configurations) evolve in a dS background, during inflation, and in a Minkowski background, after inflation. As correctly pointed out in $[23,24]$, patches in which the Higgs probes field values beyond the barrier do not necessarily end up in the AdS vacuum, as long as their evolution is driven by the stochastic quantum term. Only when classical evolution takes over, the field falls into 
its deep minimum. In $[23,24]$ it was assumed that these AdS patches rapidly evolve into relic defects that are not necessarily dangerous, hence arguing that large Higgs fluctuations do not pose a cosmological threat. In our analysis, we reach opposite conclusions.

The evolution of AdS bubbles in an inflationary dS background depends on their size, internal energy, surface tension, and initial wall velocity. Depending on the characteristics of the bubbles, we find a variety of possible evolutions. Bubbles shrink, if they start with small radius and low velocity; expand but remain hidden inside the Schwarzschild horizon, if the gravitational self-energy of their surface overwhelms the difference between the vacuum energy in the exterior and interior; and expand at the expense of exterior space, otherwise. The seemingly paradoxical situation of an expanding bubble of crunching AdS space is resolved by understanding the difference in space-time coordinates on the two sides of the wall. While an observer inside the bubbles will experience space contracting because of the negative cosmological constant, an external observer will see the surface of large bubbles expand. Although we expect that the process of inflation with large $H$ will generate a distribution of AdS expanding bubbles, we conclude that such bubbles will never take over all dS space. The inflationary space expansion always beats the causal expansion of bubbles, efficiently diluting them.

At this stage, it may seem that the remnant AdS bubbles can be compatible with the presently observed universe. The problem starts when we consider post-inflationary evolution of the AdS patches in a flat background. The bubble wall keeps on expanding at the speed of light and an AdS patch eventually engulfs all space. This means that a necessary requirement for our present universe to exist is that the probability to find an expanding AdS bubble in our past light-cone must be negligible. Unfortunately, we cannot make firm statements about the formation of expanding AdS Higgs bubbles during inflation because the answer depends on energy considerations based on the Higgs potential in the Planckian region. However, our study suggests that it would be very difficult to imagine a situation in which all large-field Higgs patches shrink and none expands. Therefore, barring the presence of AdS Higgs bubbles in our past light-cone is a well-justified requirement for a viable cosmology. This line of reasoning leads to an interesting bound on $H$, the Hubble constant during inflation, which we compute not only in the case of a minimally-coupled Higgs, but also in the presence of a gravitational interaction between the Higgs bilinear and the scalar curvature.

Having established the dangers of patches in which the Higgs field falls into the transPlanckian region, we consider the fate of patches in which, at the end of inflation, the Higgs field has fluctuated beyond the potential barrier, but has not yet experienced the classical evolution that wants to drive it towards very large values. The eventual fate of such bubbles is determined by the subsequent thermal evolution of the universe. Thermal effects can rescue such patches of the universe by effectively pushing the potential barrier to larger field values, allowing the Higgs field to relax into its SM vacuum. We study this phenomenon during the preheating and reheating stages of the universe, when the energy stored in the inflaton oscillating around its minimum is released into thermal energy carried by SM particles. In this way we can express the constraint on $H$ as a function of the reheating temperature after inflation. 
Finally, in a more speculative vein, we explore the consequences of a conjecture put forward in the context of quantum theories of gravity. It has been argued that no formulation of quantum mechanics in dS spaces can be consistent. On the other hand, we observe today a positive cosmological constant. The resolution of this conflict between a conceptual obstruction and an empirical fact can be found by assuming that the asymptotic state of our universe is not dS. In other words, we are only living in a transitory situation and today's dS space will soon terminate. Of course, there are many ways in which the universe could escape the allegedly dreadful $\mathrm{dS}$ condition, but it is tempting to speculate that the instability of the electroweak vacuum is the emergency exit chosen by nature. If we take this hypothesis seriously, we obtain a rather precise prediction for a combination of the Higgs and top masses in the SM, in good agreement with experimental measurements. The predicted strip in parameter space can be narrowed further by the hypothesis that the universe must have been sufficiently hot in the past (for instance, for allowing some high-temperature mechanism of baryogenesis).

The paper is organised as follows. In section 2 we address the preliminary technical issue of the gauge dependence of the effective potential. The generation and evolution of Higgs fluctuations during inflation is studied in section 3, while the subsequent evolution after inflation is the subject of section 4 . Our speculations on the quantum-gravity prediction of the Higgs mass are discussed in section 5, and our results are summarised in section 6. The details of the general-relativity calculation of the evolution of AdS bubbles in dS or Minkowski backgrounds are contained in the appendix.

\section{Gauge dependence of the SM effective potential}

The critical Higgs mass below which the SM Higgs potential becomes unstable is gaugeindependent; however the instability scale of the SM potential, e.g. $h_{\max }$, defined as the Higgs field value at which $V_{\text {eff }}(h)$ is maximal, is gauge-dependent (as recently emphasised in [32-34]). Therefore, one has to be cautious in extracting from the potential a physically meaningful scale associated to the instability.

There is a number of ways in which one can try to identify scales that track the potential instability and are gauge-invariant because expressed in terms of extrema. ${ }^{1}$ However, our cosmological computations will employ the full SM effective potential also away from its extrema, so we are confronted with the issue of the gauge-dependence of the effective potential shape, an old topic much debated in the literature. ${ }^{2}$ In dealing with this issue

\footnotetext{
${ }^{1}$ For example, this could be done through the scale of a higher-dimensional operator $h^{n}$ (with $n>4$ ) that, added to the SM, cures its instability.

${ }^{2}$ We summarise some of the main approaches here. Nielsen [35] proved that the gauge dependence of the effective potential can be reabsorbed by a re-definition of the fields. Tye [36] found that the effective potential and the effective kinetic terms are separately gauge-invariant if the perturbative expansion is performed by decomposing the Higgs doublet into the physical Higgs field $h$ and the 3 angular coordinates $\pi$, such that the Goldstone fields $\pi$ are massless at any value of $h$, not only at the extrema of the potential. The results then agree with the unitary gauge. Buchmuller et al. [37] computed the effective potential in terms of the gauge-invariant combination $\Phi_{H}^{\dagger} \Phi_{H}$ claiming a gauge-invariant effective potential; again this selects the radial mode of the Higgs doublet such that Goldstone are always massless. Schwartz et al. [33, 34]
} 
we follow a pragmatic approach. First, we insist on calculating physical quantities, that can be proven to be gauge independent. Second, we make sure that the approximations we use in those calculations are consistent, in the sense that any residual gauge dependence is smaller than the precision of our approximations.

The gauge-independence of our results is ultimately based on the Nielsen identity that describes how the effective action depends on the gauge-fixing parameters and how to extract out of it gauge independent quantities. Let us briefly discuss how this works.

The fact that the Higgs effective potential $V(h)$ depends on the gauge parameters (generically denoted as $\xi$ ) follows from the fact that the effective action $S_{\text {eff }}$ itself is a gauge-dependent object. In spite of this, as is well known, both the potential and the effective action are extremely useful and physical quantities extracted from them (like particle masses, $S$-matrix elements, the vacuum energy density, tunnelling rates in the case of metastable vacua, etc.) turn out to be gauge-independent, as they should.

\subsection{Gauge (in)dependence of the effective action}

The Nielsen identities [38-41] tell us that the gauge dependence of the effective action can be compensated by a local field redefinition. In other words, different gauges describe the same physics in terms of different coordinates in field space (leading to different potentials but also to different kinetic terms). Particularising to cases with Higgs background only, one has

$$
\xi \frac{\partial S_{\mathrm{eff}}}{\partial \xi}=-\int d^{4} x K[h(x)] \frac{\delta S_{\mathrm{eff}}}{\delta h(x)},
$$

where $K[h(x)]$ is a functional of $h$ that can be found in [38-41].

One immediate consequence of the Nielsen identity is that the action evaluated on a solution of the equation of motion for $h, \delta S_{\text {eff }} / \delta h=0$, is gauge-independent. We also see that the gauge-independence of the extremal values of the effective potential follows directly by applying the previous general fact to constant field configurations.

Writing the effective action in a derivative expansion

$$
S_{\mathrm{eff}}[h]=\int d^{4} x\left[-V(h)+\frac{1}{2} Z(h)\left(\partial_{\mu} h\right)^{2}+\mathcal{O}\left(\partial^{4}\right)\right],
$$

we can find a series of Nielsen identities for the coefficients of this expansion [42, 43] by expanding in the same way $K[h]$ and $\delta S_{\text {eff }} / \delta h$ in (2.1), as

$$
\begin{aligned}
K[h] & =C(h)+D(h)\left(\partial_{\mu} h\right)^{2}-\partial^{\mu}\left[\tilde{D}(h) \partial_{\mu} h\right]+\mathcal{O}\left(\partial^{4}\right), \\
\frac{\delta S_{\mathrm{eff}}}{\delta h} & =-V^{\prime}+\frac{1}{2} Z^{\prime}\left(\partial_{\mu} h\right)^{2}-\partial^{\mu}\left[Z(h) \partial_{\mu} h\right]+\mathcal{O}\left(\partial^{4}\right),
\end{aligned}
$$

where primes denote $h$-derivatives. The higher-order derivative terms are expected to be suppressed by an energy scale, which can be as low as the value of the Higgs field, and by a one-loop factor, which is the same for any order in derivatives. Therefore, our derivative

argue that finding a gauge-invariant definition of the effective potential is a misguided enterprise and that the contribution of Goldstone bosons should be neglected (at leading order) in a consistent perturbative expansion around the field value at which $\lambda=0$. 
expansion is valid only when the gradient of the Higgs field is smaller than the homogeneous value of the field under consideration.

At the lowest order in the derivative expansion, we find the expression for the gaugedependence of the effective potential

$$
\xi \frac{\partial V}{\partial \xi}+C(h) V^{\prime}=0
$$

which, as anticipated, ensures the gauge-independence of the values of the potential at its extremal points. This Nielsen identity also tells us that the explicit $\xi$-dependence of the potential can be compensated by an implicit $\xi$-dependence of the field as:

$$
\xi \frac{d h}{d \xi}=C(h),
$$

so that $d V / d \xi=0$. At order $\mathcal{O}\left(\partial^{2}\right)$ we get

$$
\xi \frac{\partial Z}{\partial \xi}=-C Z^{\prime}-2 Z C^{\prime}+2 D V^{\prime}+2 \tilde{D} V^{\prime \prime}
$$

where we suppressed the $h$ dependence of all functions.

It is useful to consider the order in weak gauge couplings (denoted generically by $g$ in this paragraph) of the different functions that appear in the previous identities [42]. As we will be interested in the potential region where the Higgs quartic coupling gets negative, eventually inducing a new minimum radiatively, we will use the counting $\lambda \sim g^{4}$. The function $C(h)$ starts at one loop and is $\mathcal{O}\left(g^{2}\right)$. The Nielsen identity (2.5) then implies that the $\xi$ dependence of $V$ starts at $\mathcal{O}\left(g^{6}\right)$. On the other hand, the Nielsen identity (2.7) implies that the $\xi$ dependence of $Z$ starts at $\mathcal{O}\left(g^{2}\right)$, with the terms involving $D$ and $\tilde{D}$ being of higher order in $g$. As we will see in the next subsection, it will be sufficient for our purposes to deal with the dominant $\xi$ dependence of the potential so that we will neglect the effect of the subleading $D$ and $\tilde{D}$ terms in what follows, as in [42].

Let us next consider the $\xi$-dependence of the equation of motion for $h$ which we write using

$$
\operatorname{EoM}[h] \equiv \sqrt{Z} \partial^{2} h+\frac{1}{2 \sqrt{Z}} Z^{\prime}\left(\partial_{\mu} h\right)^{2}+\frac{1}{\sqrt{Z}} V^{\prime},
$$

and its solutions $\bar{h}(x)$, which satisfy $\operatorname{EoM}[\bar{h}]=0$. It is straightforward to show that

$$
\left.\xi \frac{d}{d \xi} \operatorname{EoM}[h]\right|_{h=\bar{h}}=0
$$

up to $\mathcal{O}\left(\partial^{4}\right)$ corrections, provided we use $\xi d \bar{h} / d \xi=C$. In principle, one can continue the check of the gauge invariance of the equations of motion iteratively up to infinite order in the number of derivatives. ${ }^{3}$ This means that, if some $\bar{h}_{\xi}(x)$ solves the equation of motion for some choice of $\xi$ and we shift $\xi \rightarrow \xi+d \xi$, the shifted solution is $\bar{h}_{\xi}(x)+d \bar{h}_{\xi}(x)$ with $d \bar{h}_{\xi}(x)=C\left(\bar{h}_{\xi}\right) d \xi / \xi$. In other words, the field rescaling that can balance the effect of

\footnotetext{
${ }^{3}$ Using the previous identities one can also check, to all orders, the $\xi$-independence of the scalar physical mass $M_{h}^{2} \equiv V^{\prime \prime} /\left.Z\right|_{\min }$, evaluated at the minimum of the potential, as indicated.
} 
changing $\xi$ in the effective potential is the same field rescaling that applies to the solutions of the equation of motion for different $\xi$.

The same rescaling works for the Fokker-Planck and Langevin equations that we will use later on to describe the Higgs fluctuations during inflation. These equations take the form, LANGEvin $\left[h_{L}\right]=0$ and FokkerPlanck $[P(h, t)]=0$, with

$$
\text { LANGEVIN }[h] \equiv \sqrt{Z} \frac{d h}{d t}+\frac{1}{3 H \sqrt{Z}} V^{\prime}-\eta,
$$

and

$$
\text { FoKkerPlanck }[P(h, t)] \equiv \frac{1}{\sqrt{Z}} \frac{\partial}{\partial h}\left\{\frac{1}{\sqrt{Z}}\left[\frac{\partial}{\partial h}\left(\frac{H^{3}}{8 \pi^{2}} \frac{P}{\sqrt{Z}}\right)+\frac{1}{3 H} \frac{P V^{\prime}}{\sqrt{Z}}\right]\right\}-\frac{1}{\sqrt{Z}} \frac{\partial P}{\partial t} .
$$

Here $P(h, t) d h$ is the probability for finding the Higgs field in the infinitesimal interval between $h$ and $h+d h$ at time $t$ during inflation. The fact that $P$ is a probability density explains why $P$ enters in eq. (2.11) through the ratio $P / \sqrt{Z}$.

Using these expressions and the $\xi$-dependence of $V(h), Z(h)$, and $h$ as described in eqs. (2.5)-(2.7), we get

$$
\left.\frac{d}{d \xi} \operatorname{LANGEVin}[h]\right|_{h=h_{L}}=0,\left.\quad \frac{d}{d \xi} \operatorname{FoKkERPLANCK}[P(h, t)]\right|_{P=\bar{P}}=0,
$$

(where $h_{L}$ and $\bar{P}$ are solutions of the Langevin and Fokker-Planck equations, respectively) up to corrections that can be shown to be subleading. ${ }^{4,5}$ This shows once again that if we have a solution of the Langevin equation for a given value of $\xi$, we automatically obtain a solution for $\xi+d \xi$ by the shift $h(\xi)+C(h(\xi)) d \xi / \xi$. For the Fokker-Planck equation, a solution for general $Z$ is formally related to a solution for $Z=1$ again by a field rescaling, with $P(h, t) / \sqrt{Z(h)}=P_{c}\left(h_{c}(h)\right)$, where the relation between $h$ and the canonical field $h_{c}$ follows from $d h_{c} / d h=\sqrt{Z(h)}$. As a result, the integrated probability is independent of the field rescaling:

$$
\int_{h_{c, i}}^{h_{c, f}} P_{c}\left(h_{c}\right) d h_{c}=\int_{h_{i}}^{h_{f}} P_{c}\left(h_{c}(h)\right) \sqrt{Z(h)} d h=\int_{h_{i}}^{h_{f}} P(h) d h .
$$

\footnotetext{
${ }^{4}$ Here we are explicitly using the derivative expansion previously introduced to derive the gauge transformation properties of $V, Z$ and $h$. Indeed, the Langevin and Fokker-Planck formalism represent a truncation of the theory at the lowest order in derivatives, where the approximation is justified by the smallness of the gradient of the field with respect to the Hubble parameter. In the rest of the paper, we will be using these equations to describe evolutions of the Higgs field for values of the Hubble parameters even quite larger than the Higgs vev itself. For this reason, we cannot naively apply the zeroth order truncation in derivatives of the effective action, because, as we discussed, the derivative expansion is suppressed only by the Higgs vev. However, since the higher derivative corrections are suppressed by at least a one-loop factor and are not log-enhanced (at one-loop), a consistent truncation is to use the Langevin and Fokker-Planck equations as derived from an effective action where the only corrections that are included are the non-derivative, leading log-enhanced, ones. This will be how we will use the Langevin and Fokker-Planck equations in the rest of the paper.

${ }^{5}$ Note also that, concerning the dependence on the renormalisation scale $\mu$, one can show the $\mu$ independence of $M_{h}^{2}, \operatorname{EoM}[h], \operatorname{Langevin}[h]$ and FoKkerPlancK $[P(h, t)]$ just making use of $d V / d \mu=0$, $d h / d \log \mu=\gamma h, d(\partial V / \partial h) / d \log \mu=-\gamma(\partial V / \partial h), d Z / d \log \mu=-2 \gamma Z$, etc.
} 
This implies that the probability of finding the field beyond $h_{\max }$ after a given number of efolds is a gauge invariant quantity: although the value of $h_{\max }$ depends on $\xi$, the $\xi$-change of the ratio $P(h, t) / \sqrt{Z(h)}$ corresponds to the same field-rescaling $h(\xi) \rightarrow h(\xi)+C(h(\xi)) d \xi / \xi$ and leaves the integrated probabilities unchanged.

To sum up, the key idea is that a change in a given gauge parameter $\xi$ is equivalent to a redefinition of the Higgs field, which should leave physics invariant. The effective potential, the equations of motion for the Higgs field and the Fokker-Planck and Langevin equations enjoy a sort of "covariance" under changes of the gauge parameters. The equations are changed in such a way that the change induced in their solutions is just a common field redefinition dictated by the Nielsen identity.

\subsection{Effective potential including only log-enhanced corrections}

For the previous appealing properties to hold, the interplay between the effective potential and the kinetic term in the effective action is crucial. For the SM case at very large field values we write

$$
\mathscr{L}_{\text {eff }}=Z(h, \xi) \frac{\left(\partial_{\mu} h\right)^{2}}{2}-\lambda_{\text {eff }}(h, \xi) \frac{h^{4}}{4}+\cdots
$$

where the ellipsis denotes higher derivative terms and both $Z(h, \xi)$ and $\lambda_{\text {eff }}(h, \xi)$ include radiative corrections and depend on $\xi$. As usual, it proves convenient to use a canonically normalised Higgs field $h_{\text {can }}(h, \xi)$ as $d h_{\text {can }} / d h=Z^{1 / 2}$ and to re-express the effective Lagrangian in terms of $h_{\text {can }}$, obtaining

$$
\mathscr{L}_{\text {eff }}=\frac{\left(\partial_{\mu} h_{\text {can }}\right)^{2}}{2}-\lambda_{\text {can }}\left(h_{\text {can }}, \xi\right) \frac{h_{\text {can }}^{4}}{4}+\cdots
$$

In terms of the canonical field all the equations become simpler as we do not have to drag the $Z$ factor around. An additional bonus is that the residual $\xi$ dependence in our approximations will be significantly reduced. Let us see how this works examining the gauge dependence of the effective potential. The coloured dashed curves in figure 1 show $\lambda_{\text {eff }}(h, \xi)$, which tracks the large field behaviour of the SM effective potential, as computed at next-to-leading order (NLO) accuracy in the Fermi $\xi$ gauges $[32]^{6}$ further improved by performing a resummation of IR-divergent Goldstone loops $[44,45] .^{7}$ We take into account the running of $\xi_{1}$ and $\xi_{2}$ (gauge-fixing parameters for hypercharge and $\mathrm{SU}(2)_{L}$ ), assuming a common value $\xi$ renormalised at $M_{t}$. We confirm that $\lambda_{\text {eff }}(h, \xi)$ significantly depends on the gauge-parameter $\xi$. The black curves in figure 1 show $\lambda_{\text {can }}\left(h_{\text {can }}, \xi\right)$, again computed at NLO in Fermi $\xi$ gauges: we see that the dependence on $\xi$ almost completely disappeared - all black curves almost merged into a single curve.

One can explain analytically why the gauge dependence approximately cancels out by looking at the dominant corrections enhanced by large logarithms, which are resumed by solving the RG equations and setting the RG scale $\bar{\mu}$ around the field value of interest:

$$
\lambda_{\mathrm{eff}}(h, \xi) \approx e^{4 \Gamma(\bar{\mu} \approx h, \xi)} \lambda(\bar{\mu} \approx h), \quad Z_{\mathrm{eff}}(h, \xi) \approx e^{2 \Gamma(\bar{\mu} \approx h, \xi)},
$$

\footnotetext{
${ }^{6}$ At this level of approximation the potential has a residual dependence on the RG scale $\bar{\mu}$ comparable to the gauge dependence. We here adopted the choice $\bar{\mu}=h e^{\Gamma}$ that minimises the error.

${ }^{7}$ Around the minimum of the potential, this resummation becomes equivalent to the expansion of [33, 34], which makes the truncation of the potential compatible with the Nielsen identity.
} 

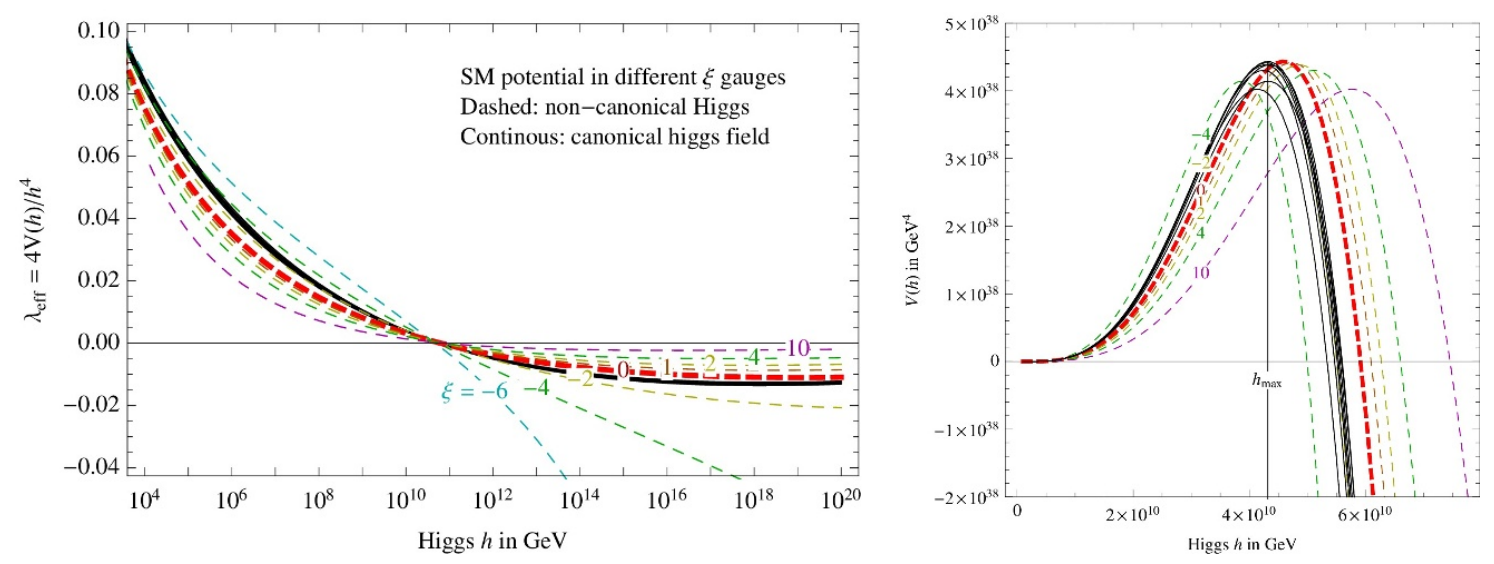

Figure 1. The dashed curves show the effective quartic coupling (left) and effective SM potential (right) computed at next-to-leading order in a generic Fermi $\xi$-gauge. The thick red dashed curve corresponds to the Landau gauge, $\xi=0$. The right handed panel shows that the height of the potential barrier is only approximately gauge-independent (a measure of the residual gauge dependence). The black continuous curves show the same potential expressed in terms of the canonical field $h_{\text {can }}$ : the gauge dependence in the potential gets compensated by the gauge-dependence of the kinetic term, such that the continuous curves nearly overlap.

where $\Gamma=\int_{M_{t}}^{\bar{\mu}} \gamma d \ln \bar{\mu}, \gamma$ is the gauge-dependent anomalous dimension of the Higgs field, and $\lambda(\bar{\mu})=\int_{M_{t}}^{\bar{\mu}} \beta_{\lambda} d \ln \bar{\mu}$ is the running quartic coupling, where $\beta_{\lambda}$ is gauge-independent. In this leading-order (LO) approximation one has

$$
h_{\text {can }} \approx h e^{\Gamma(\bar{\mu} \approx h)}, \quad \lambda_{\text {can }}\left(h_{\text {can }}\right) \approx \lambda(\bar{\mu} \approx h),
$$

which is gauge-independent because the RGE for $\lambda$ and all other couplings of the theory are gauge-independent. The order-of-magnitude gauge dependence of $h_{\max }$ found in [32] disappears because it is almost entirely due to the RG factor $\Gamma$.

Such LO cancellation has been noticed before, see e.g. [46, 47]. Note however that the field redefinition dictated by the Nielsen identity we discussed earlier and the field redefinition required to make the field canonical are the same only at LO. Moreover, the field redefinition from the Nielsen identity becomes considerably more complicated at NLO [35]. Its use to define a "gauge-independent" potential is in fact equivalent to choosing a particular gauge and therefore does not solve the problem of how to extract gauge-invariant quantities out of the effective action. For this reason we refrain from attempting to use it as a way of defining a gauge-invariant potential and simply use the canonical field as a way of reducing the residual gauge dependence of our results.

The previous discussion has been carried out in Fermi gauge at NLO to help us clarify the issues related to gauge invariance. Having understood them, we can now use the state-of-the-art computation of the effective potential in the Landau gauge $(\xi=0)$ with NNLO accuracy (2 loop finite corrections and 3 loop RGE corrections) [1-3] combined with the use of a field redefinition to make the field canonical (taking into account the effect of potentially large logarithms in $Z$ ). Using that canonically normalised Higgs field $h$, in the region around the top of the barrier, the SM Higgs potential can be analytically 
approximated as

$$
V_{\mathrm{eff}}(h) \approx-b \ln \left(\frac{h^{2}}{h_{\max }^{2} \sqrt{e}}\right) \frac{h^{4}}{4},
$$

where $h_{\max }$ is the field value at which $V_{\text {eff }}(h)$ takes its maximal gauge-invariant value $V_{\text {eff }}\left(h_{\max }\right)=b h_{\max }^{4} / 8$. Using the value $b \approx 0.16 /(4 \pi)^{2}$ for the $\beta$ function of $\lambda$ around $h_{\max }$, we find $h_{\max }=5 \times 10^{10} \mathrm{GeV}$ for the present best-fit values of $M_{t}, M_{h}$ and $\alpha_{3}$. Although this value of $h_{\max }$ is computed in Landau gauge and it would be slightly different in other gauges, the reader should keep in mind that the results we present in the following sections are gauge-invariant even if for convenience we express them in terms of $h_{\max }{ }^{8}$

\section{Higgs fluctuations during inflation}

The instability of the Higgs potential leads to an interesting dynamics during inflation. We focus on the relevant radial mode $h=\sqrt{2\left|\Phi_{H}\right|^{2}}$ of the Higgs doublet. If the Hubble constant $H$ is large enough, $h$ fluctuates beyond the potential barrier. If the true vacuum is deep enough, inflation stops in the regions where the Higgs falls, while inflation continues in the (possibly rare) regions where accidentally $h<h_{\max }$. In this section we compute the probability of the possible outcomes at the end of inflation, while in the next section we will discuss what happens after inflation.

In the absence of a large Higgs mass term, the evolution of the long wavelength modes of the $h$ field is controlled by the Langevin equation [48]

$$
\frac{d h}{d t}+\frac{1}{3 H} \frac{d V(h)}{d h}=\eta(t)
$$

where $\eta$ is a Gaussian random noise with

$$
\left\langle\eta(t) \eta\left(t^{\prime}\right)\right\rangle=\frac{H^{3}}{4 \pi^{2}} \delta\left(t-t^{\prime}\right)
$$

It is important to realise that eq. (3.1) is valid only if the positive effective mass squared $V^{\prime \prime}(h)$ of the Higgs field is light enough compared to $H^{2}$. Only under these circumstances the long wavelength super-Hubble fluctuations of the Higgs field are generated. On the contrary, if $V^{\prime \prime}(h)>9 H^{2} / 4$, the resulting power spectrum of Higgs fluctuations is both suppressed by $\exp \left(-2 V^{\prime \prime}(h) / H^{2}\right)$ and by the fact that the spectrum is strongly tilted on the blue side [49-51]. ${ }^{9}$

\subsection{Higgs fluctuations during inflation for $\xi_{H}=0$}

It is convenient to rewrite eq. (3.1) replacing time $t$ with the number of $e$-folds $N=H t$, and to normalise the Higgs field and its potential in units of the Higgs value $h=h_{\max }$ at

\footnotetext{
${ }^{8}$ Alternatively, we could choose other scales associated (more indirectly) to the instability which are explicitly gauge invariant. One could be the renormalisation scale $\mu_{0}$ at which the quartic Higgs coupling $\lambda$ crosses zero; another choice is the scale $\mu_{X}$ at which the one-loop radiatively corrected Goldstone mass is zero (as used in [33, 34]). For the same central values above we get $\mu_{0}=1 \times 10^{10} \mathrm{GeV}$ and $\mu_{X}=4.5 \times 10^{10} \mathrm{GeV}$.

${ }^{9}$ Indeed, the solution of the Klein-Gordon equation for a spin 0 particle with mass $m$ in de Sitter goes like $\exp (-\pi \mu / 2)(-\tau)^{3 / 2} H_{i \mu}(-k \tau)$, where $\mu=\sqrt{m^{2} / H^{2}-9 / 4}$ and $\tau$ is the conformal time.
} 


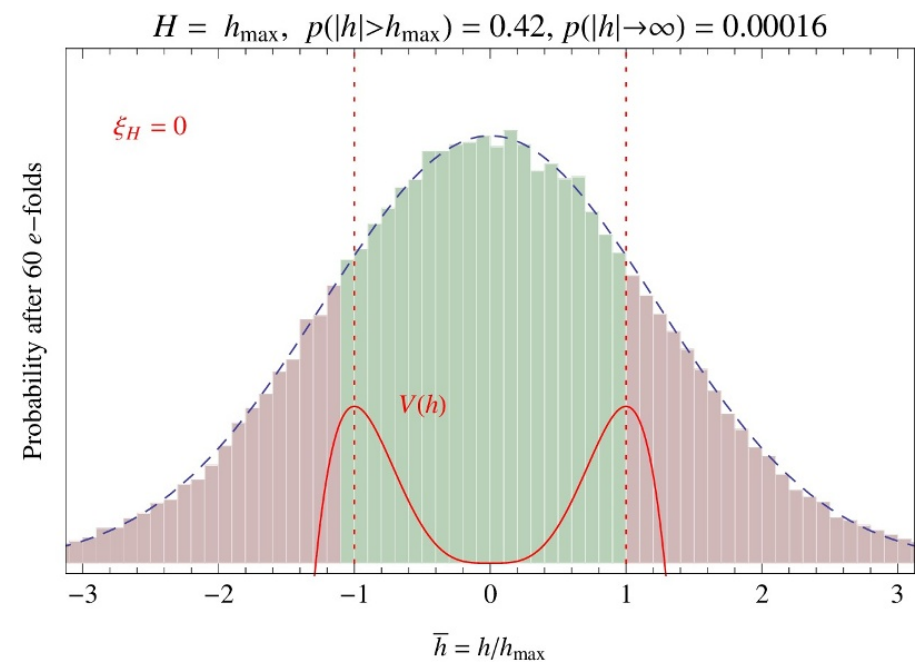

Figure 2. Random distribution of the Higgs field $\bar{h}=h / h_{\max }$ after $N=60$ e-folds of inflation with Hubble constant equal to the Higgs instability scale, $H=h_{\max }$. The blue dashed curve is the $V=0$ Gaussian approximation of eq. (3.8). The red curve is the SM Higgs potential $\bar{V}(\bar{h})$, in arbitrary units.

which $V\left(h_{\max }\right)=V_{\max }$ is maximal,

$$
\bar{h}=\frac{h}{h_{\max }} \quad \text { and } \quad \bar{V}(\bar{h}) \equiv \frac{V}{h_{\max }^{4}} \approx-b \ln \left(\frac{\bar{h}^{2}}{\sqrt{e}}\right) \frac{\bar{h}^{4}}{4} .
$$

After these redefinitions, the Langevin equation in eq. (3.1) becomes

$$
\frac{d \bar{h}}{d N}+\frac{h_{\max }^{2}}{3 H^{2}} \frac{d \bar{V}(\bar{h})}{d \bar{h}}=\bar{\eta}(N)
$$

where the noise $\bar{\eta}(N)$ obeys

$$
\left\langle\bar{\eta}(N) \bar{\eta}\left(N^{\prime}\right)\right\rangle=\left(\frac{H}{2 \pi h_{\max }}\right)^{2} \delta\left(N-N^{\prime}\right) .
$$

One can now numerically generate random realisations of the Higgs evolution in $N$, in steps of $d N$, as

$$
\bar{h}(N+d N)=\bar{h}(N)-\frac{h_{\max }^{2}}{3 H^{2}} \bar{V}^{\prime}(\bar{h}) d N+r
$$

where $r$ are random numbers extracted from a Gaussian distribution with zero mean and standard deviation $\sigma=H \sqrt{d N} /\left(2 \pi h_{\max }\right)$.

Indeed, for $h \gg \sigma$, the same result is reproduced by the analytic solution to the FokkerPlanck equation for the probability $P(h, N)$ of finding the Higgs field at the value $h$ after $N$ e-folds of inflation,

$$
\frac{\partial P}{\partial N}=\frac{\partial^{2}}{\partial h^{2}}\left(\frac{H^{2}}{8 \pi^{2}} P\right)+\frac{\partial}{\partial h}\left(\frac{V^{\prime}}{3 H^{2}} P\right),
$$

taking $V=0$ and boundary conditions at $h= \pm \infty$. 


$$
N=60 e \text {-folds, } \xi_{H}=0
$$

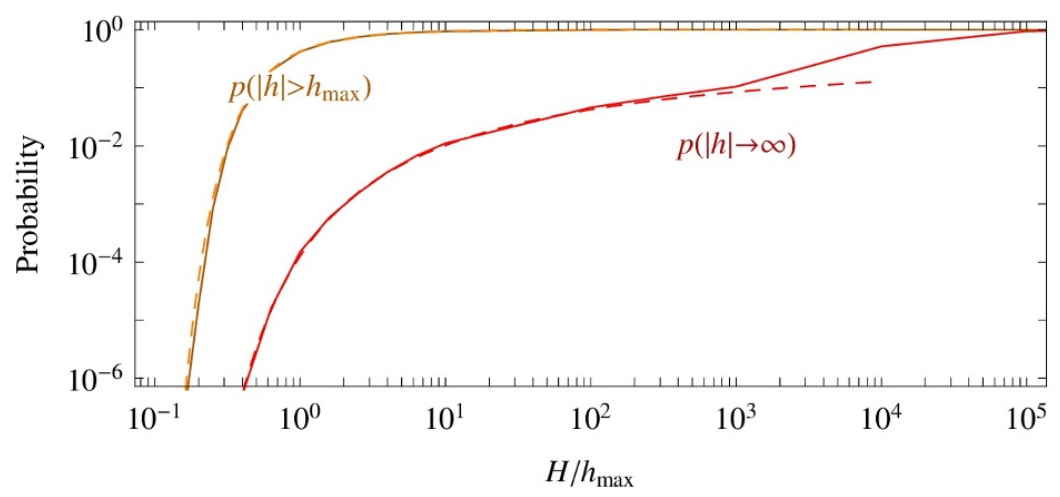

Figure 3. Minimal probability that, after $N=60$ e-folds of inflation, the Higgs fluctuated above the SM potential barrier (orange curve), or fall down to the true minimum (red curve). The continuous curves are the numerical results; the dashed curves are the analytical approximations presented in the text.

Figure 2 shows the resulting probability density of the value of $h$ after $N=60$ efoldings, starting from $\bar{h}=0$ at the beginning of inflation. The result, a quasi-Gaussian distribution, has a simple interpretation. Given that the quartic Higgs coupling vanishes around the instability scale, for a large range of Higgs values around the instability scale the classical evolution (sourced by the gradient of the potential) is negligible with respect to the quantum evolution (sourced by the random noise $\eta$ ). As a consequence, even assuming that the Higgs starts from $h=0$, the field $h$ acquires a Gaussian distribution with zero mean and variance that grows with $N$ :

$$
P(h, N)=\frac{1}{\sqrt{2 \pi\left\langle h^{2}\right\rangle}} \exp \left(-\frac{h^{2}}{2\left\langle h^{2}\right\rangle}\right), \quad \sqrt{\left\langle h^{2}\right\rangle}=\frac{H}{2 \pi} \sqrt{N} .
$$

The distribution shown in figure 2 maintains its quasi-Gaussian shape also for values of the Higgs field well above $h_{\max }$. Therefore, during inflation, the Higgs field can fluctuate above the barrier without being sucked into the negative-energy (AdS) true vacuum. The only regions where the Higgs falls into the true minimum are those where $h$ fluctuates to field values so large that the potential slope can no longer be neglected.

Regions that fluctuate above the potential barrier. The minimal probability that the Higgs ends up beyond the top of its potential barrier after $N$ e-folds is

$$
p\left(|h|>h_{\max }\right) \approx 1-\operatorname{erf}\left(\frac{\sqrt{2} \pi h_{\max }}{\sqrt{N} H}\right) .
$$

This probability, obtained by integrating the Gaussian distribution for $|h|>h_{\max }$, is minimal because it corresponds to the initial condition $h=0$. Shifting the peak of the distribution to a non-vanishing value of $h$ will only increase $p\left(|h|>h_{\max }\right)$. The solid orange curve in figure 3 shows our numerical result for this probability as a function of the Hubble constant during inflation, in units of the Higgs instability scale $H / h_{\max }$. The dashed orange 
curve corresponds to the analytic expression in eq. (3.9), which is evidently an excellent approximation.

No constraints arise if, after inflation, the regions with $|h|>h_{\max }$ fall back to the SM minimum, pushed by thermal effects (see section 4). If instead, after inflation, the regions with $|h|>h_{\max }$ fall down into the true AdS minimum, then their probability should be smaller than $e^{-3 N}$, so that it is unlikely to find the Higgs away from its EW vacuum in any of the $\sim e^{3 N}$ causally independent regions that are formed during inflation and that constitute the observable universe today. Using $1-\operatorname{erf}(x) \simeq e^{-x^{2}} / \sqrt{\pi} x$ for large $x$, this condition implies

$$
\frac{H}{h_{\max }}<\sqrt{\frac{2}{3}} \frac{\pi}{N} \approx 0.04
$$

Regions that fall to the true minimum during inflation. The approximation of neglecting the scalar potential $V$, which led to the quasi-Gaussian distribution of the Higgs field values, breaks down at large $h$. There, the gradient of the potential dominates over quantum fluctuations, and $h$ falls down to its true minimum already during inflation. The solid red curve in figure 3 shows our numerical result for such probability.

We can analytically estimate the probability for $h$ to fall into its true vacuum after $N$ e-folds of inflation. We first consider a potential $V=\lambda h^{4} / 4$ with constant $\lambda$ and assume that the bulk of the Higgs field probability distribution is still given by the Gaussian in eq. (3.8), cut at large field values. The location of the cut is estimated by demanding that the classical evolution becomes more important than the quantum fluctuations [23, 24]. This can be quantified by requiring that the second term in the right-hand side of eq. (3.7) dominates over the first one,

$$
\left|\frac{\partial}{\partial h}\left(\frac{V^{\prime}}{3 H^{2}} P\right)\right|>k\left|\frac{\partial^{2}}{\partial h^{2}}\left(\frac{H^{2}}{8 \pi^{2}} P\right)\right|
$$

where $k$ is a fudge factor and $P$ is given in eq. (3.8). Equation (3.11) implies that the Gaussian distribution must be cut for $h^{2}>3 k H^{2} /(2|\lambda| N)$, and values of $h$ that satisfy this inequality are sucked into the true minimum.

Therefore, the probability of falling to infinity is exponentially suppressed for small $|\lambda|$ :

$$
p(|h| \rightarrow \infty) \approx 1-\operatorname{erf}\left(\frac{\pi \sqrt{3 k}}{N \sqrt{|\lambda|}}\right)
$$

Such probability satisfies $p(|h| \rightarrow \infty)<e^{-3 N}$ for $|\lambda|<k \pi^{2} / N^{3}$.

Considering now the more realistic case of the SM potential with a running coupling $\lambda(h)=-b \ln \left(h^{2} / h_{\max }^{2} \sqrt{e}\right)$, we find

$$
p(|h| \rightarrow \infty) \approx 1-\operatorname{erf}\left(\frac{\pi \sqrt{3 k}}{N \sqrt{b B}}\right), \quad \text { where } \quad B=\operatorname{PL}\left(\frac{3 k H^{2}}{2 b N h_{\max }^{2}}\right)
$$

where PL is the ProductLog function. This analytic approximation of $p(|h| \rightarrow \infty)$ is shown in figure 3 as the dashed red line and agrees well with the numerical computation, once we fit the fudge factor to be $k=2.6$. Next, we need to extrapolate the analytic approximation to 


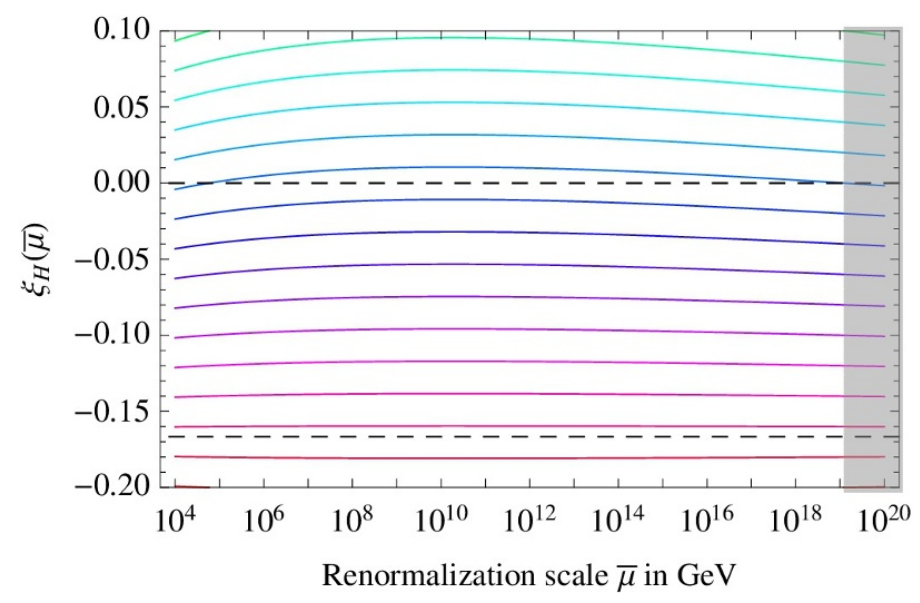

Figure 4. Running of the Higgs coupling to gravity $\xi_{H}$ as a function of the renormalisation scale in the SM, for different initial conditions at the Planck scale. The dashed horizontal lines correspond to the special values $\xi_{H}=-1 / 6$ and $\xi_{H}=0$.

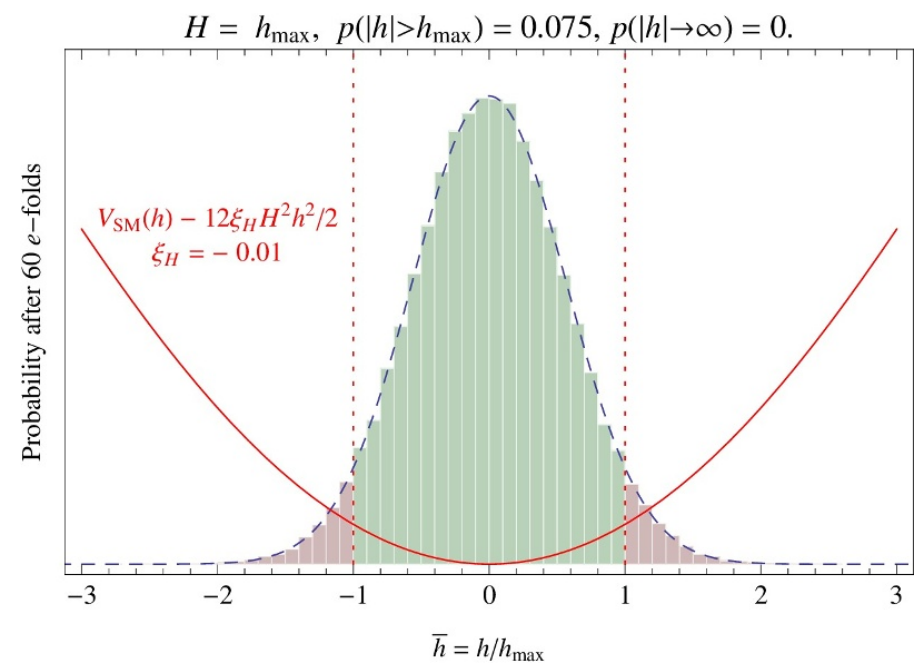

Figure 5. Random distribution of the Higgs field $\bar{h}=h / h_{\max }$ after $N=60$ e-folds of inflation with Hubble constant equal to the Higgs instability scale, $H=h_{\max }$, and for $\xi_{H}=-0.01$. The blue dashed line is the Gaussian approximation of eq. (3.19). The red curve is the Higgs potential $V_{\mathrm{SM}}(h)-12 \xi_{H} H^{2} h^{2} / 2$, in arbitrary units.

probabilities much smaller than those that can be computed numerically. The probability $p(|h| \rightarrow \infty)$ is smaller than $e^{-3 N}$ for

$$
\frac{H}{h_{\max }}<\frac{\pi}{N} \sqrt{\frac{2}{3}} e^{\pi^{2} k / 2 b N^{3}} \approx 0.045
$$

\subsection{Higgs fluctuations during inflation for $\xi_{H} \neq 0$}

Higgs fluctuations during inflation can get damped if the Higgs doublet $\Phi_{H}$ during inflation acquires an effective mass $m$. Various effects can contribute to such mass: 


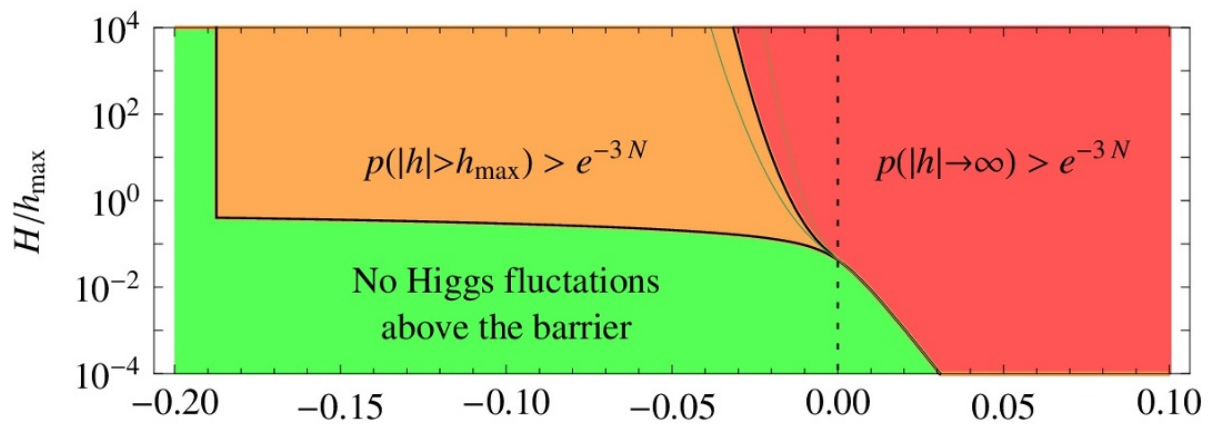

Higgs coupling to gravity $\xi_{H}$

Figure 6. As a function of $\xi_{H}$ and the Hubble constant in units of the instability scale $h_{\max }$ (and for $N=60 e$-folds of inflation), we show the three regions where: the probability for the Higgs field to end up in the negative-energy true minimum is larger than $e^{-3 N}$ (red); the probability for the Higgs field to fluctuate beyond the potential barrier is larger than $e^{-3 N}$ (orange); the latter probability is smaller than $e^{-3 N}$ (green). Higgs fluctuations are damped for $\xi_{H}<-3 / 16$. The uncertainty on the orange/red boundary corresponds to a fudge factor $1 / 3<k<3$.

1. a quartic term $\lambda_{h \phi}\left|\Phi_{H}\right|^{2} \phi^{2}$ in the potential, which describes a coupling between the Higgs $\Phi_{H}$ and the inflaton $\phi$, generates during inflation an extra contribution $m^{2}=$ $\lambda_{h \phi} \phi^{2}$ to the Higgs mass;

2. a decay of the inflaton into SM particles can generate a non-vanishing temperature during inflation. Such decay are kinematically blocked when SM particles acquire a thermal mass larger than the inflaton mass, of order $H$. Thereby the Higgs can acquire a mass $m^{2} \approx H^{2}$

3. a non-minimal Higgs coupling to gravity, $-\xi_{H}\left|\Phi_{H}\right|^{2} R$ contributes as $m^{2}=\xi_{H} R=$ $-12 \xi_{H} H^{2}$.

These contributions to $\mathrm{m}^{2}$ would have qualitatively similar effects. In our quantitative analysis we focus on the latter effect because the presence of the $\xi_{H}$ term is unavoidable: even if $\xi_{H}=0$ at some energy scale, SM quantum corrections generate a non-vanishing value of $\xi_{H}$ at any other energy scale. Indeed, ignoring gravity, the one-loop running of $\xi_{H}$ is given by

$$
\frac{d \xi_{H}}{d \ln \bar{\mu}}=\frac{\xi_{H}+1 / 6}{m^{2}} \frac{d m^{2}}{d \ln \bar{\mu}}=\frac{\xi_{H}+1 / 6}{(4 \pi)^{2}}\left(6 y_{t}^{2}-\frac{9}{2} g_{2}^{2}-\frac{9}{10} g_{1}^{2}+12 \lambda_{H}\right)+\cdots
$$

where $\bar{\mu}$ is the renormalisation scale. The RGE for the Higgs mass parameter $m^{2}$ is known up to 3 loops in the $\overline{\mathrm{MS}}$ scheme (as summarised in [1-3]), and we have shown here only the leading term. The SM couplings are such that $d \ln \left|m^{2}\right| / d \ln \bar{\mu}$ is positive (negative) at energy roughy below (above) $10^{10} \mathrm{GeV}$. The evolution of $\xi_{H}$ for different boundary conditions at $M_{\mathrm{Pl}}$ is shown in figure 4 ; it has a fixed point at the conformal value $\xi_{H}=-1 / 6$. Notice that this value is not special for our analysis because it does not recover conformal invariance, which is broken at the level of the SM Higgs effective potential. 
We consider the following action

$$
S=\int d^{4} x \sqrt{g}\left[-\frac{\bar{M}_{\mathrm{Pl}}^{2}}{2} R-\xi_{H}\left|\Phi_{H}\right|^{2} R+\left|D_{\mu} \Phi_{H}\right|^{2}-V+\cdots\right]
$$

where $V \simeq V(\phi)+\lambda\left|\Phi_{H}\right|^{4}$ is the scalar potential of the Higgs and of the inflation $\phi$ and $\bar{M}_{\mathrm{Pl}}$ is the reduced Planck mass. We use the approximation that, during inflation, the inflaton potential is constant $V(\phi) \simeq V_{I}{ }^{10}$

The $\xi_{H}$ coupling of the Higgs to gravity affects the scalar potential during inflation by inducing an effective Higgs mass term $m^{2}=\xi_{H} R=-12 \xi_{H} H^{2} \simeq-4 \xi_{H} V_{I} / \bar{M}_{\mathrm{Pl}}^{2}$ which can stabilise the Higgs potential and suppress Higgs fluctuations. As explained after eq. (3.1), Higgs fluctuations are damped if $\xi_{H}<-3 / 16$. For $-3 / 16<\xi_{H}<0$, Higgs fluctuations are still present, but become less dangerous than in the case of vanishing $\xi_{H}$.

Neglecting the small Higgs quartic coupling, adding the effective Higgs mass term $m^{2}=-12 \xi_{H} H^{2}$, and assuming the ansatz of a Gaussian distribution with variance $\left\langle h^{2}\right\rangle$,

$$
P(h, N)=\frac{1}{\sqrt{2 \pi\left\langle h^{2}\right\rangle}} \exp \left(-\frac{h^{2}}{2\left\langle h^{2}\right\rangle}\right)
$$

the evolution of $\left\langle h^{2}\right\rangle$ is obtained from the Fokker-Planck equation (3.7) and becomes, at $h^{2} \gg\left\langle h^{2}\right\rangle$

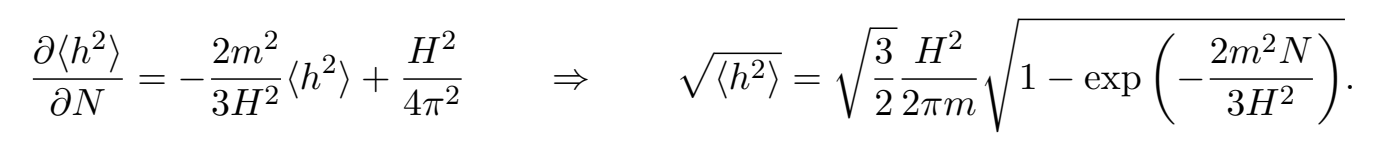

If $m^{2}<0$, the variance grows exponentially with $N$. If $m^{2}>0$, the Higgs probability distribution approaches, after a few $e$-folds, the limiting distribution given by ${ }^{11}$

$$
\sqrt{\left\langle h^{2}\right\rangle}=\frac{H}{4 \pi \sqrt{-2 \xi_{H}}} .
$$

This is to be compared with eq. (3.8), which holds for $\xi_{H}=0$. Figure 5 shows a numerical example: already for $\xi_{H}=-0.01$ the variance is significantly reduced.

Using eq. (3.19), we obtain the following bounds on $H$ from the request that the probabilities of the Higgs fluctuating beyond the barrier $\left(|h|>h_{\max }\right)$ or falling into the true minimum $(|h| \rightarrow \infty)$ are less than $e^{-3 N}$ :

$$
\begin{aligned}
p\left(|h|>h_{\max }\right)<e^{-3 N} & \Rightarrow \frac{H}{h_{\max }}<4 \pi \sqrt{\frac{-\xi_{H}}{3 N}}, \\
p(|h| \rightarrow \infty)<e^{-3 N} & \Rightarrow \frac{H}{h_{\max }}<4 \pi \sqrt{\frac{-\xi_{H}}{3 N}} e^{32 \pi^{2} \xi_{H}^{2} / b N} .
\end{aligned}
$$

\footnotetext{
${ }^{10}$ Of course, one could also envisage other operators coupling the Higgs field with gravity, e.g. $\left|\Phi_{H}\right|^{2} R^{2} / M_{\mathrm{Pl}}^{2}$. However, in most models of inflation, the Hubble parameter squared decreases linearly with the number of $e$-folds $N_{e}$ till the end of inflation. Therefore, for $\xi_{H} \gtrsim\left(H_{I}^{2} / M_{\mathrm{Pl}}^{2}\right)\left(N_{e} / N_{I}\right)$, where $H_{I}$ is the initial value of the Hubble rate when inflation starts and $N_{I}$ is the total number of $e$-folds, the higher-order operator becomes negligible. This condition becomes easier and easier to satisfy as inflation proceeds.

${ }^{11}$ This is larger than what is obtained by naively assuming a Hawking temperature $T=H / 2 \pi$.
} 
These bounds are the analogues of eqs. (3.10) and (3.14), which are valid for $\xi_{H}=0$. An order-one fudge factor $k$ can be similarly introduced such that eq. (3.21) closely agrees with the numerical result; however $k$ depends on $\xi_{H}$ and thereby differs from what we previously discussed in the limit $\xi_{H}=0$. Taking into account how $\xi_{H}$ stabilises the potential results in a more complicated, but numerically similar, analytic expression. Furthermore these approximations needs to be extrapolated down to probabilities smaller than those that can be compared to the numerical result. Conservatively estimating the uncertainty by varying $1 / 3<k<3$, in figure 6 we summarise the situation by showing the regions of $\xi_{H}$ and $H / h_{\max }$ where the bounds in eq.s (3.20) and (3.21) are satisfied for $N=60$.

In the presence of a $\lambda_{h \phi}\left|\Phi_{H}\right|^{2} \phi^{2}$ potential coupling between the Higgs $\Phi_{H}$ and the inflaton $\phi$, during inflation one has an extra contribution to the Higgs mass, $m^{2}=\lambda_{h \phi} \phi^{2}$. This term has a similar effect as the inflationary mass discussed above. The Higgs $h$ has no inflationary fluctuations as long as $m>3 H / 2$. However, in general $m^{2}$ changes during inflation in a model-dependent way. Considering, for example, large-field inflation with a quadratic potential, one has $\phi=2 \bar{M}_{\mathrm{Pl}} \sqrt{N_{I}-N}$ during inflation, where $N_{I} \gtrsim 60$ is the total number of $e$-folds. Inserting $m^{2}=4 \lambda_{h \phi} \bar{M}_{\mathrm{Pl}}^{2}\left(N_{I}-N\right)$ into eq. (3.18) one finds that the maximal Higgs fluctuation is achieved at the end of inflation and is Planck suppressed:

$$
\left\langle h^{2}\right\rangle=\sqrt{\frac{3}{\pi \lambda_{h \phi}}} \frac{H^{3}}{16 \pi \bar{M}_{\mathrm{Pl}}} .
$$

\subsection{Bubble evolution in de Sitter spacetime}

After having computed the probability for inflationary fluctuations to form regions where the Higgs field lies at its true minimum, the next question we have to address is how these regions evolve. In the literature one finds conflicting statements about the evolution of AdS regions in an inflationary background. One point of view, based on flat-space intuition, is that AdS regions should expand because their interior has lower energy than the exterior. A different point of view is that, since AdS space eventually contracts, regions in which the Higgs lies at its true minimum will shrink, possibly leaving some almost point-like relics, which are nevertheless efficiently diluted, and thus made harmless, by the inflationary expansion of space. We will show that addressing the question about the fate of AdS regions involves a number of non-trivial and counter-intuitive issues raised by general relativity.

First, gravitational energy contributes to the total energy budget. Second, an AdS region might expand, while remaining hidden behind a black-hole horizon. Third, the interior AdS space is dynamically unstable [25]: when described in cosmological FRW coordinates it reaches a 'big-crunch' singularity in a finite amount of internal time of order $\left(G V_{\text {in }}\right)^{-1 / 2}$, where $-V_{\text {in }}<0$ is the internal cosmological constant. If space is empty, this is just a coordinate singularity (AdS can be continued using better coordinates); if space is filled by a background field (for example the Higgs field), its fluctuations grow until the energy density becomes infinite and a physical singularity appears. Furthermore, the AdS geometry has a timelike boundary, such that the evolution cannot be predicted after the bubble wall reaches the boundary, unless additional boundary conditions are imposed there (in other words, information must flow in from infinity). As a result, a Cauchy 
horizon appears in the interior of the bubble. It is expected that, within the full theory beyond the thin-wall limit, a physical spacelike singularity must develop before the Cauchy horizon [52]. This confirms the expectation that the AdS bubble is unstable.

In order to clarify all these issues we performed a careful (and somewhat lengthy) general-relativistic computation, described in appendix A. Here we summarise the main points.

In order to make the problem tractable analytically, we assume a spherical AdS region (that we thereby call 'bubble'), separated from the outside space by a thin wall with constant surface tension $\sigma$. The matching of the external and internal geometries requires the presence of such a wall with nonzero energy density. The fate of the AdS bubble is then determined by computing the motion of the wall separating the AdS interior from the external space (de Sitter during inflation and Minkowski after inflation). In the thin-wall approximation, the motion of the wall is determined by junction conditions that relate the extrinsic curvature on each of its sides [53]. The bubbles that we now compute are more general than those that arise from vacuum decay with zero total energy, already studied in [25]. The basic elements of our calculation are the following.

1. The space inside the bubble is assumed to be an empty spherical region of AdS space with metric

$$
d s^{2}=-f_{\text {in }}(r) d \eta^{2}+\frac{d r^{2}}{f_{\text {in }}(r)}+r^{2} d \Omega_{2}^{2}, \quad r<R,
$$

expressed in global coordinates. Here $f_{\text {in }}(r)=1+r^{2} / \ell_{\text {in }}^{2}$ is the usual AdS solution, with vacuum energy $-V_{\text {in }}$ corresponding to the length scale $1 / \ell_{\text {in }}^{2}=8 \pi G V_{\text {in }} / 3$.

2. The space outside the bubble is described by the metric

$$
d s^{2}=-f_{\text {out }}(r) d t^{2}+\frac{d r^{2}}{f_{\text {out }}(r)}+r^{2} d \Omega_{2}^{2}, \quad r>R,
$$

where $f_{\text {out }}(r)=1-r^{2} / \ell_{\text {out }}^{2}-2 G M / r$ describes a Schwarzschild-de Sitter (SdS) spacetime, with $G=1 /\left(8 \pi \bar{M}_{\mathrm{Pl}}^{2}\right)$. Here $M$ is the mass of the bubble as seen by an outside observer, living in an asymptotically de Sitter space described by the length scale $1 / \ell_{\text {out }}^{2}=8 \pi G V_{\text {out }} / 3=H^{2}$. As discussed later, the metric in eq. (3.24) also describes the case of the asymptotically flat spacetime produced after inflation, which is obtained in the limit $V_{\text {out }} \rightarrow 0$, so that $f_{\text {out }}(r)=1-2 G M / r$.

Note that, for $\ell_{\text {out }} \gg G M$, the SdS spacetime contains two horizons, corresponding to the zeros of $f_{\text {out }}(r)$ : the inner (Schwarzschild) horizon at $r \approx 2 G M$ and the outer (de Sitter) horizon at $r \approx \ell_{\text {out }}$. The corresponding Penrose diagram is depicted in the right panel of figure 7. It is a combination of the diagrams for the Schwarzschild and de Sitter spacetimes [27]. Thick blue lines denote curvature singularities, the dashed lines horizons and the dotted lines conformal infinities. The two thin vertical lines at the ends of the diagram indicate that the pattern is repeated indefinitely on either side. 
3. The two regions are separated by a domain wall with constant surface tension $\sigma$. The metric on the domain wall can be written as

$$
d s^{2}=-d \tau^{2}+R^{2}(\tau) d \Omega_{2}^{2},
$$

where $R(\tau)$ denotes the location of the wall in both coordinate systems (3.23) and (3.24). The evolution is expressed in terms of the proper time $\tau$ on the wall. In the full problem, $\sigma$ is given by the kinetic and potential energy of the Higgs field, and is different for each Higgs configuration. Within our approximation, all energy stored in the Higgs potential goes into the motion of the wall, leaving the AdS interior empty.

The detailed calculation described in appendix A shows that the (naively positive) difference between the energy in the exterior $\left(\ell_{\text {out }}^{-2}\right)$ and the interior $\left(-\ell_{\text {in }}^{-2}\right)$ of the bubble that controls whether the bubble expands or contracts receives a gravitational correction $-\kappa^{2}$, so that the relevant parameter is the quantity:

$$
\Delta=\frac{1}{\ell_{\mathrm{in}}^{2}}+\frac{1}{\ell_{\mathrm{out}}^{2}}-\kappa^{2}, \quad \kappa \equiv 4 \pi G \sigma .
$$

As discussed after eq. (A.11), the contribution $\sim \kappa^{2}$ can be interpreted, from a Newtonian point of view, as the gravitational self-energy of the wall. The motion of the wall can be described as the Newtonian motion of a point particle

$$
\left(\frac{d \tilde{R}}{d \tilde{\tau}}\right)^{2}+V(\tilde{R})=E
$$

in an effective 'potential' given by

$$
V(\tilde{R})=-\left(\frac{1+\epsilon \tilde{R}^{3}}{\tilde{R}^{2}}\right)^{2}-\frac{\gamma^{2}}{\tilde{R}}-\delta^{2} \tilde{R}^{2}
$$

where $\tilde{R}=\rho R$ is a rescaled dimensionless coordinate that describes the position of the wall as a function of a rescaled dimensionless proper time $\tilde{\tau}=2 \kappa \tau / \gamma^{2}$. The various constants are given by

$$
\delta^{2}=\frac{4 \kappa^{2}}{\ell_{\text {out }}^{2} \Delta^{2}}, \quad \rho^{3}=\frac{|\Delta|}{2 G M}, \quad \epsilon \equiv \operatorname{sign} \Delta, \quad \gamma=\frac{2 \kappa}{\sqrt{|\Delta|}}, \quad E=-\frac{\kappa^{2}}{G^{2} M^{2} \rho^{4}} .
$$

The possible types of bubble evolution are discussed in detail in appendix A. The complete analysis is performed for an asymptotically flat exterior spacetime, for which there are fewer cases. The evolution within an asymptotically dS spacetime does not display any novel characteristics, and is discussed more briefly. The study of the 'potential' shows that there are two cases in which bubbles do not expand: either they start small enough, or their expansion is hidden behind a black-hole horizon (this possibility corresponds to $\Delta<0$ ). We will discuss in section 4.3 if any of these possibilities is realised in the simpler case of Higgs bubbles in an external flat spacetime, after the end of inflation. 

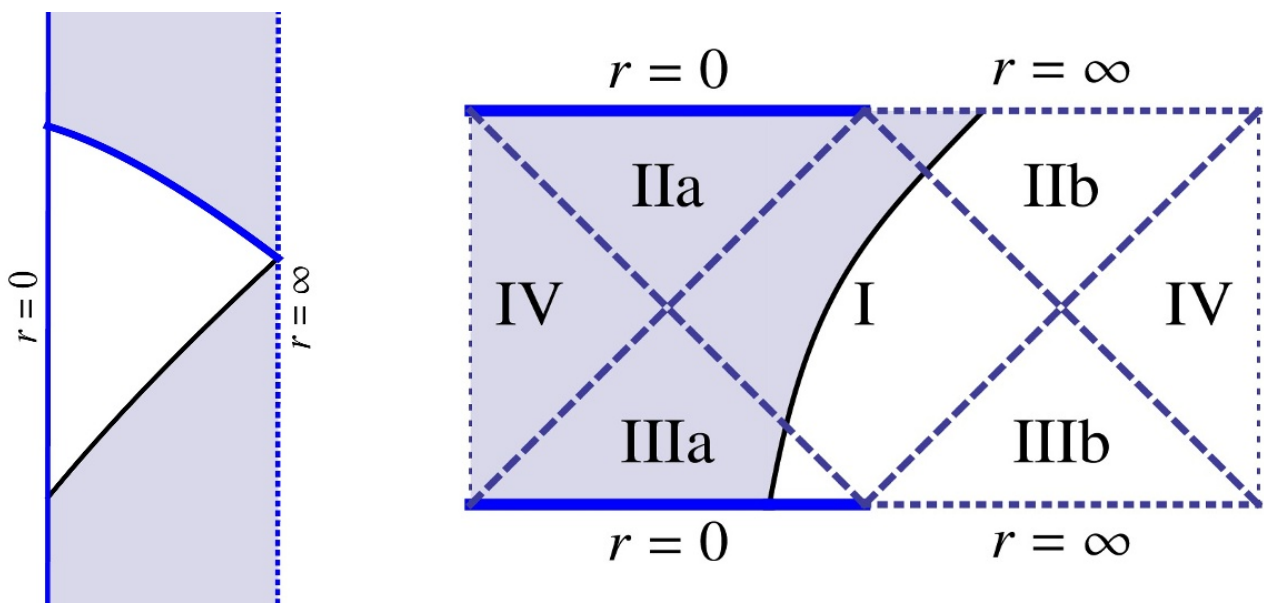

Figure 7. Penrose diagram describing an AdS bubble that expands in a dS space. The black curve denotes the thin wall separating the two phases; the true space is obtained by patching the region in white in the left panel (AdS interior of the bubble) with the region in white in the right panel (Schwarzschild-dS exterior of the bubble). The dashed lines denote the various horizons, while the thick blue line in the left panel denotes the AdS singularity ('crunch').

The standard evolution of sufficiently large bubbles is characterised by expansion, with their wall crossing the outer (dS) horizon. A typical example is presented in the Penrose diagram of figure 7: the wall starts below the inner horizon and subsequently expands, passing through both horizons and eventually reaching a speed close to that of light. The bubble grows in size and takes over part of the dS spacetime. The crucial question is whether the bubble can engulf the total exterior spacetime, thus ending inflation. It is apparent from figure 7 that this does not happen. Asymptotically the AdS bubble replaces only part of the spacelike surface $r=\infty$, with the remaining dS space remaining unaffected. In other words, expanding bubbles are inflated away. Inflation precisely has the purpose of splitting the expanding universe into causally disjoint regions, and this limits the effect of the bubble growth: bubbles expand, but the dS space between them also grows. In the limit of infinite inflation, both the bubbles and the exterior de Sitter phase acquire infinite extent.

We can estimate the asymptotic bubble size through the use of dS planar coordinates, commonly employed in the study of inflation. The metric has the form

$$
d s^{2}=-d t_{p}^{2}+e^{2 H t_{p}}\left(d r_{p}^{2}+r_{p}^{2} d \Omega_{2}^{2}\right) .
$$

Assuming that the wall follows an almost null trajectory, we find that its location is given by

$$
r_{p}=r_{p 0}+\frac{1}{H}\left(1-e^{-H t_{p}}\right) .
$$

Bubbles are created within the causally connected region, which extends up to $1 / H$ at $t_{p}=0$. This means that its typical radius $r_{p 0}$ is of order $1 / H$. Its subsequent growth 

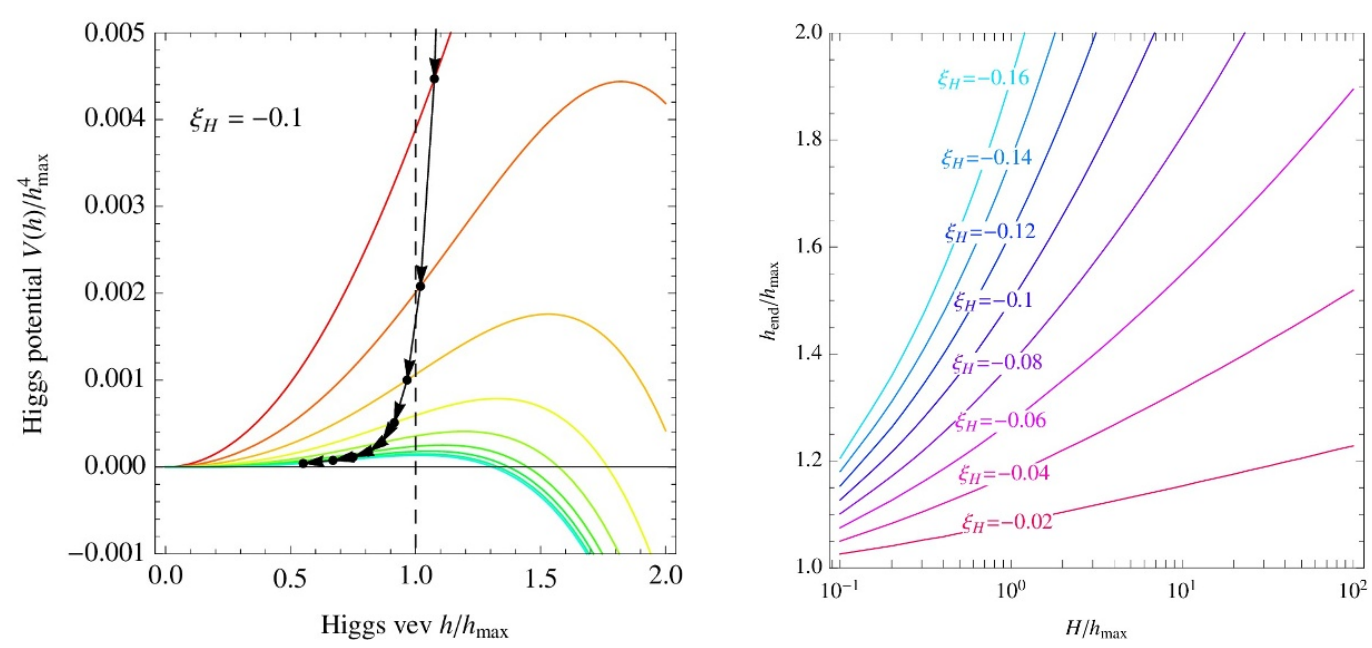

Figure 8. Left: an example of how the dynamical evolution during pre-heating can bring back the Higgs field into the stable region before the instability takes over. The coloured lines show the potential at successive intervals of time, and the black line shows the trajectory of the Higgs field. Right: maximal value of the Higgs field at the end of inflation $\left(h_{\mathrm{end}}\right)$ that is brought back by a non-minimal gravitational coupling $\xi_{H}$ into the stable region, $h<h_{\max }$, shown as a function of the Hubble constant during inflation and for different values of $\xi_{H}$.

extends this radius by $1 / H .{ }^{12}$ It is reasonable then to expect that during inflation a typical bubble can be created with a certain probability within a causally connected region and then will roughly follow the general expansion of this region outside the horizon. It cannot, however, engulf the whole spacetime. The picture is completely different for an asymptotically flat exterior. As we shall see in the next section, in that case an expanding bubble can take over the whole spacetime.

Another important question concerns the consequences for an outside observer of the AdS 'crunch' in the bubble interior. We discuss this issue in detail in appendix A.5. From the point of view of an observer deep inside the bubble, the coordinates in which the bubble appears as homogenous are those of an expanding and subsequently contracting open FRW universe with constant negative energy density. The bubble wall can be roughly identified with the $\hat{t}=0$ surface in this slicing (see figure 19 in the appendix). After a finite (and short) time $\hat{t}$, of order the AdS radius, a singularity forms in the bubble interior. However, this singularity never reaches the wall, as the latter expands with the speed of light. On the other hand, from the point of view of an external observer the bubble just expands forever (within either de Sitter or Minkowski spacetime).

\section{Higgs evolution after inflation}

In this section we study the evolution of the Higgs field after inflation, considering that inflation ends with a matter-dominated phase, characterised by inflaton oscillations, followed by the reheating process, which ignites the usual thermal phase characterised by a gas of SM particles.

\footnotetext{
${ }^{12}$ Note, however, that the physical bubble radius is obtained after multiplication by the divergent factor $\exp \left(H t_{p}\right)$.
} 


\subsection{Higgs evolution during pre-heating}

We start by considering the pre-heating phase, during which the energy density of the universe is dominated by the inflaton oscillations around its minimum. The interest of this phase lies in the case in which the Higgs potential has an extra mass term $\frac{1}{2} m^{2} h^{2}$ induced either by a non-minimal coupling to gravity $\left(m^{2}=-12 \xi_{H} H^{2}\right)$ or by a coupling to the inflaton $\phi\left(m^{2}=\lambda_{h \phi} \phi^{2}\right)$. In either case, the mass term rapidly shuts off after inflation. However, we will show that the induced $m^{2}$ can still have an important stabilising effect during the pre-heating phase.

Let us suppose that, at the end of inflation, the universe enters a matter-dominated phase, where the equation of state is that of a pressure-less gas, which is a good approximation when the inflaton field is oscillating before reheating. In this case the effective mass of the Higgs field is $m^{2}=-3 \xi_{H} H_{\mathrm{m}}^{2}$ and the Hubble rate scales as $H_{\mathrm{m}}=H / a^{3 / 2}$ where we have set the value of the scale factor $a$ at the end of inflation to unity. ${ }^{13}$

We consider a region in which, once inflation ends, the Higgs field has the value $h_{\text {end }}$. If the Higgs mass term during inflation $\left(m^{2}=-12 \xi_{H} H^{2}\right)$ is larger than $(9 / 4) H^{2}$ (i.e. if $\left.\xi_{H}<-3 / 16\right)$, then the Higgs field is anchored at the origin and $h_{\mathrm{end}}=0$. We are interested here in the opposite regime, when $-3 / 16<\xi_{H}<0$ and quantum fluctuations of the Higgs are generated during inflation; in this case, $h_{\mathrm{end}}$ is generally not zero. The subsequent evolution of the Higgs field is governed by the equation

$$
\frac{d^{2} h}{d t^{2}}+3 H_{\mathrm{m}}(t) \frac{d h}{d t}+\frac{\partial V}{\partial h}=0 \quad \Rightarrow \quad \frac{d^{2} h}{d a^{2}}+\frac{5}{2 a} \frac{d h}{d a}+\frac{a}{H^{2}} \frac{\partial V}{\partial h}=0 .
$$

Keeping only the mass term in $V$ and neglecting the quartic term, we find $\left(a / H^{2}\right) \partial V / \partial h=$ $-3 \xi_{H} h / a^{2}$ and then the solution of eq. (4.1) is

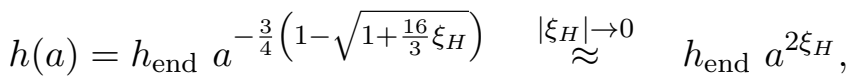

where we have neglected the solution with $h \propto a^{-3 / 2}$, which is rapidly damped with respect to eq. (4.2), and where the last approximation is valid only for $\left|\xi_{H}\right| \ll 3 / 16$.

As the amplitude of the Higgs field and the contribution to the potential from the $m^{2}$ term are both decreasing in time, we need to investigate if the Higgs field has time enough to roll down to the safe region $h<h_{\max }$ before the instability starts to become more important than the fading $m^{2}$ term, reverting the evolution of $h$. An example of the Higgs field behaviour is shown in figure 8a. The time at which the instability starts driving the Higgs dynamics can be estimated by requiring that the quartic term in the scalar potential $\left(\lambda h^{4} / 4\right.$ with $\left.\lambda=-b \ln h^{2} / h_{\max }^{2} \sqrt{e}\right)$ is comparable with the mass term $\left(m^{2} h^{2} / 2\right.$ with $\left.m^{2}=-3 \xi_{H} H^{2} / a^{3}\right)$ at $h_{\max }$, which corresponds to

$$
a^{3}=a_{\max }^{3} \approx-\frac{12 \xi_{H} H^{2}}{b h_{\max }^{2}} .
$$

\footnotetext{
${ }^{13}$ We recall that the Ricci scalar is given by $R=-6\left(\ddot{a} / a+\dot{a}^{2} / a^{2}\right)$ for a spatially flat universe. During inflation $a=\exp (H t)$ with $H$ constant, hence $R=-12 H^{2}$. In a matter-dominated phase $\left(a \propto t^{2 / 3}\right)$ we have $R=-3 H_{\mathrm{m}}^{2}$ with $H_{m}=\dot{a} / a \propto a^{-3 / 2}$, while $R=0$ in the radiation-dominated phase $\left(a \propto t^{1 / 2}\right)$.
} 
Thereby, the instability is avoided if

$$
h_{\mathrm{end}} \lesssim h_{\max } a_{\max }^{-2 \xi_{H}} .
$$

This estimate is confirmed by the result of a numerical computation illustrated in figure 8b, in which eq. (4.1) is solved using the full SM potential.

The qualitative conclusion, whenever $\xi_{H} \neq 0$, is the following. Higgs field values that, at the end of inflation, are even a factor of $\mathcal{O}(2)$ above the instability scale $h_{\text {max }}$ are brought back into the metastability region $\left(h<h_{\max }\right)$ and saved from collapse into the AdS vacuum by the non-minimal gravitational coupling $\xi_{H}$ during pre-heating dynamics. The bound on the Hubble scale during inflation in eqs. (3.20) and (3.21) are correspondingly weakened by an $\mathcal{O}(2)$ factor. On the other hand, in regions where the Higgs remains in the instability region $h\left(a_{\max }\right) \gtrsim h_{\max }$ during pre-heating, the field quickly falls down into its deep minimum, in a time $t \sim 4 \pi / h_{\max }$, unless a large enough temperature prevents the collapse, as we discuss in the following section.

The dynamics discussed above is similar to the one in which the Higgs field is coupled to the inflaton field by a coupling of the form $\lambda_{\phi h} \phi^{2} h^{2} / 2$ that generates a contribution $m^{2}=\lambda_{\phi h} \phi^{2}$ to the Higgs mass. As discussed in section 3.1, if during inflation $m>3 H / 2$ Higgs inflationary perturbations are suppressed and the Higgs is efficiently anchored at $h=0$. If instead $m<3 H / 2$, Higgs fluctuations are generated as in eq. (3.22) and they may pose a threat. After the end of inflation, when the inflaton field oscillates and its amplitude is redshifted away as $\phi \sim a^{-3 / 2}$, the effective mass squared of the Higgs, $m^{2}=\lambda_{h \phi} \phi^{2}$, decreases as $a^{-3}$, exactly as the $m^{2}$ induced by the $\xi_{H}$ coupling $\left(m^{2}=-3 \xi_{H} H_{\mathrm{m}}^{2}\right)$. One can therefore deduce the dynamics upon identifying the two $m^{2}$. Of course, if the coupling of the inflaton field with the Higgs field is of a different nature, e.g. a non-renormalisable coupling of the form $\phi^{4} h^{2} / M_{\mathrm{Pl}}^{2}$, one needs to account for the different behaviour of the Higgs effective mass.

\subsection{Higgs evolution during reheating}

In this section we study how the reheating process affects the bounds on the Hubble constant $H$ during inflation. Indeed, the dynamical evolution during the thermal phase can bring back the Higgs field towards the EW vacuum, even in regions where $h$ has fluctuated beyond the instability barrier $\left(h \gtrsim h_{\max }\right)$ at the end of inflation. As a result, the bounds on $H$ derived in section 3 are effectively relaxed.

At the end of inflation, the energy density of the universe is dominated by the coherent oscillations of the inflaton field $\phi$ with energy density $\rho_{\phi}(t)$. The oscillations of $\phi$, started at time $t \sim 1 / H$, give a matter-dominated stage that gradually ends at $t \sim 1 / \Gamma_{\phi}$, where $\Gamma_{\phi}$ is the inflaton decay width. The decay of the inflaton field into light degrees of freedom, which quickly thermalise via SM interactions giving rise to an energy density $\rho_{R}(t)$, initiates the radiation-dominated era of the universe. The process is described by the equations

$$
\left\{\begin{array}{l}
\frac{d \rho_{\phi}}{d t}=-3 H_{r} \rho_{\phi}-\Gamma_{\phi} \rho_{\phi} \\
\frac{d \rho_{R}}{d t}=-4 H_{r} \rho_{R}+\Gamma_{\phi} \rho_{\phi}
\end{array}\right.
$$



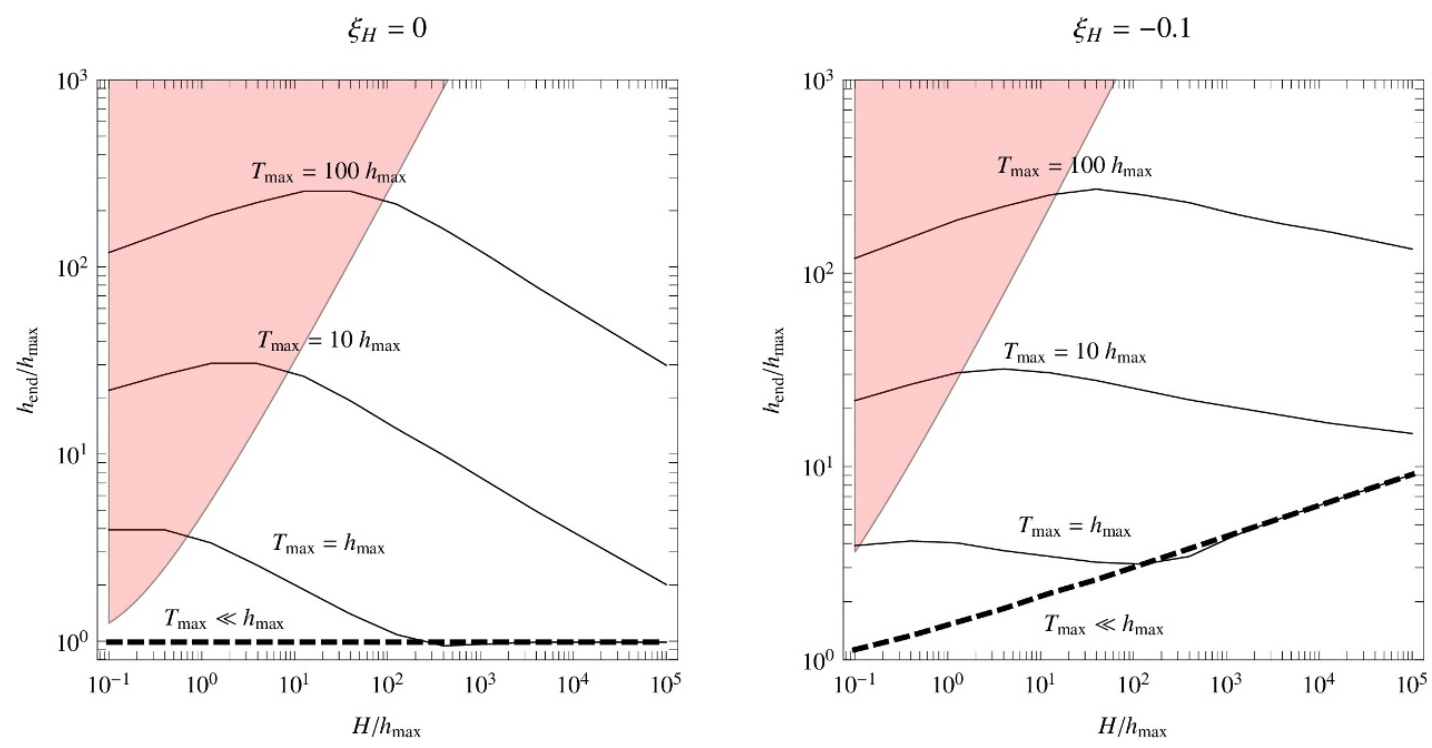

Figure 9. Maximal value of the Higgs field at the end of inflation $h_{\text {end }}$ that is brought back to the stable region, $h<h_{\max }$, as a function of the Hubble constant during inflation, for different values of $T_{\max }$ and $\xi_{H}$. This result, presented only in terms of ratios, negligibly depends on the absolute value of the instability scale $h_{\max }$, which suffers from large uncertainties mainly due to the top quark mass. In the red region the inflationary fluctuations typically drive the Higgs to its negative-energy minimum, and therefore the corresponding values of $h_{\text {end }}$ and $H$ are a highly unlikely outcome of inflation. In the parameter region below the dashed line, the field $h$ rolls towards the SM vacuum, even in the absence of any thermal effect.

where $H_{r}=\dot{a} / a=\sqrt{8 \pi\left(\rho_{\phi}+\rho_{R}\right) /\left(3 M_{\mathrm{Pl}}^{2}\right)}$ is the time-dependent Hubble constant during reheating.

The solution for the time evolution of $\rho_{\phi}$ is

$$
\rho_{\phi}(t)=\frac{\rho_{\phi}(0)}{a^{3}(t)} e^{-\Gamma_{\phi} t}, \quad \rho_{\phi}(0)=\frac{3 H^{2} M_{\mathrm{Pl}}^{2}}{8 \pi}
$$

where the initial condition $\rho_{\phi}(0)$ is given by the total energy density at the end of inflation. The second equation in the system (4.5) can be more conveniently written as

$$
\frac{d R}{d a}=\frac{\gamma a^{3 / 2} \Phi}{\sqrt{\Phi+R / a}}, \quad R \equiv \rho_{R} a^{4}, \quad \Phi \equiv \rho_{\phi} a^{3}, \quad \gamma \equiv \sqrt{\frac{\pi^{2} g_{*}}{30}} T_{\mathrm{RH}}^{2},
$$

where $g_{*}$ is the number of degrees of freedom in the thermal bath $\left(g_{*}=106.75\right.$ in the SM) and $T_{\mathrm{RH}}$ is the temperature of the system once all the inflaton energy is converted into thermal energy at the decay time,

$$
T_{\mathrm{RH}}=\left(\frac{45}{4 \pi^{3} g_{*}}\right)^{1 / 4} M_{\mathrm{Pl}}^{1 / 2} \Gamma_{\phi}^{1 / 2} .
$$

Equation (4.7) can be approximately solved at the early stage of reheating $\left(t \ll \Gamma_{\phi}^{-1}\right)$, by taking $e^{-\Gamma_{\phi} t} \approx 1$ in eq. (4.6) and neglecting the thermal-energy contribution to $H_{r}$ 
$(R / a \ll \Phi)$. Once we express $\rho_{R}$ in terms of the effective temperature $T$,

$$
\rho_{R}(t) \equiv \frac{\pi^{2} g_{*}}{30} T^{4}(t)
$$

the solution of eq. (4.7), at early times, gives the evolution of the temperature $T$ (valid till the universe enters the radiation-dominated phase)

$$
T \approx k_{1} T_{\max } a^{-3 / 8}\left(1-a^{-5 / 2}\right)^{1 / 4}, \quad T_{\max }=k_{2}\left(\frac{H M_{\mathrm{Pl}} T_{\mathrm{RH}}^{2}}{g_{*}^{1 / 2}}\right)^{1 / 4},
$$

where $k_{1}=2^{6 / 5} 3^{-3 / 20} 5^{-1 / 4}=1.3$ and $k_{2}=(3 / 8)^{2 / 5}\left(5 / \pi^{3}\right)^{1 / 8}=0.54$.

The temperature $T$ of the SM-particle gas raises from 0 to the maximum value $T_{\max }$ as long as, soon after inflation, the scale factor of the universe $a$ grows by an order-one factor in a time $t \sim 1 / H$. After reaching $T_{\max }$, the temperature decreases as $a^{-3 / 8}$, signalling the continuous release of entropy from the decay of the inflaton field. When this energy release ends, at time $t \sim 1 / \Gamma_{\phi}$, the temperature is equal to $T_{\mathrm{RH}}$, which is called the reheating temperature, and then radiation cools in the standard way, $T \propto 1 / a$, due to space expansion. Note that the entire reheating process can be described by only two parameters, which we choose to be $H$ and $T_{\mathrm{RH}}$. The decay of the Higgs condensate at the end of inflation has been discussed in ref. [54, 55].

Let us now consider the evolution of the Higgs field throughout the thermal phase. Because of thermal corrections, the Higgs potential receives an extra mass term $\frac{1}{2} m_{T}^{2} h^{2}$, where $m_{T} \sim g T$ and $g$ represents the relevant combination of coupling constants. This expression for the thermal mass holds up to field values $h \lesssim 2 \pi T$. The thermal corrections to the potential can be approximated as [56]

$$
V_{T} \approx\left(0.21-0.0071 \log _{10} \frac{T}{\mathrm{GeV}}\right) T^{2} \frac{h^{2}}{2} e^{-\frac{h^{2}}{(2 \pi T)^{2}}},
$$

where we added an exponential cut-off at high values of $h . V_{T}$ helps in stabilising the Higgs potential by shifting the instability to higher scales, in much the same way as the mass term due to the coupling $\xi_{H}$ does.

Figure 9 shows the maximum allowed value of $h_{\text {end }}$ in order for the Higgs not to fall into its true vacuum at (or above) Planckian field values. A direct comparison with the right panel of figure 8 shows that, for high enough reheating temperatures, thermal effects are indeed of extreme relevance.

It is not difficult to understand the behaviour of the maximum allowed value of $h_{\text {end }}$ as a function of the Hubble rate. Let us consider for simplicity the case $\xi_{H}=0$ (left panel in figure 9). From eq. (4.1) one can see that in a time scale of the order of the Hubble time, when the scale factor changes by order unity and the maximum temperature has been reached, the Higgs field changes by an amount (we neglect factors of order unity)

$$
h-h_{\mathrm{end}} \simeq-\frac{1}{H^{2}} V^{\prime}\left(h_{\mathrm{end}}\right) \simeq-\frac{m_{T}^{2}\left(T_{\mathrm{max}}\right) h_{\mathrm{end}}}{H^{2}}-\frac{\lambda h_{\mathrm{end}}^{3}}{H^{2}},
$$



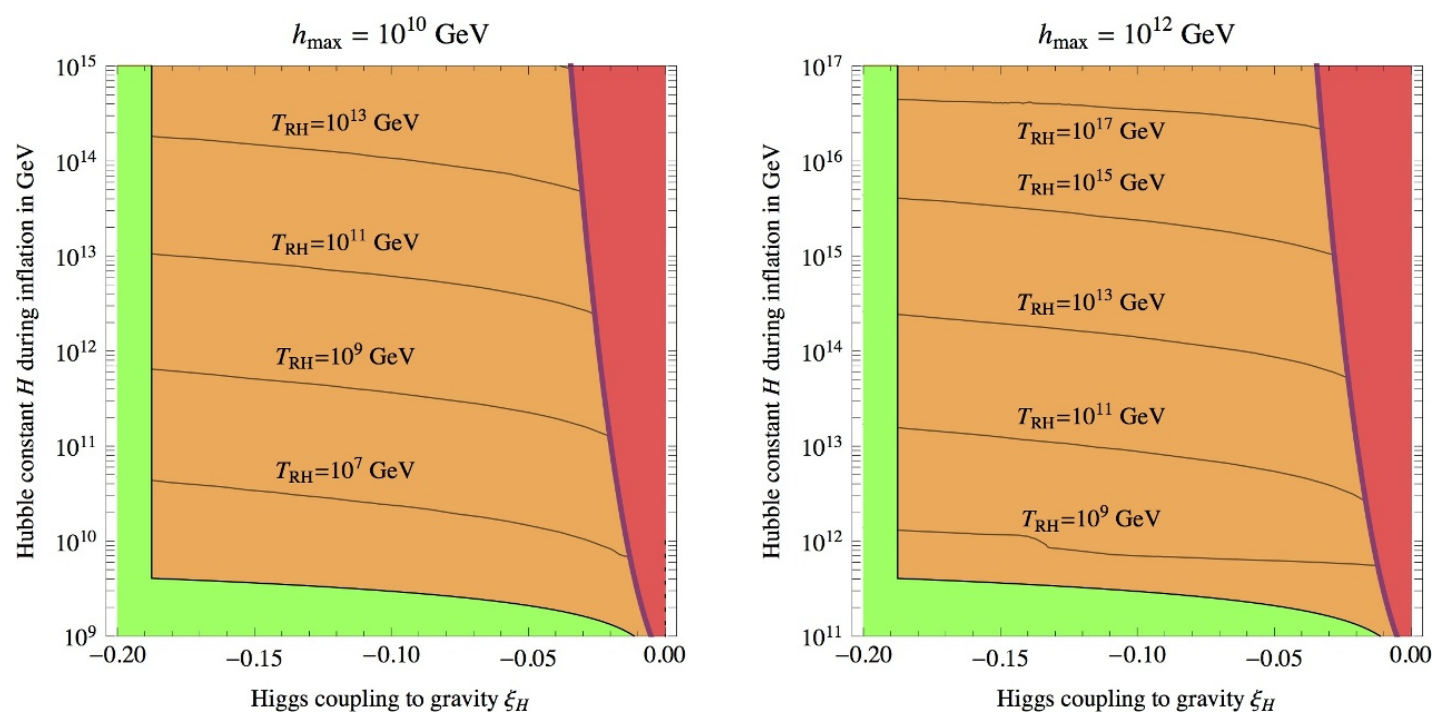

Figure 10. Minimal reheating temperature $T_{\mathrm{RH}}$ needed to prevent the fall of the Higgs down into its deep true vacuum, assuming two different values for the instability scale $h_{\max }$ of the Higgs potential.

where we have approximated the zero temperature potential as $\lambda h^{4} / 4$ and one has to remember we are considering the region where $\lambda<0$. Therefore, we obtain the approximate expression, valid soon after inflation,

$$
h \simeq h_{\mathrm{end}}\left(1-\frac{T_{\mathrm{max}}^{2}}{H^{2}}-\lambda \frac{h_{\mathrm{end}}^{2}}{H^{2}}\right) .
$$

For $h$ to roll towards the origin, a necessary condition is that the right-hand side of eq. (4.13) is smaller than one, which implies $h_{\mathrm{end}} \lesssim T_{\max } /|\lambda|^{1 / 2}$. This explains the approximate flatness of the curves in the right panel of figure 9 for small $H$. For $H \gg T_{\max }$, the approximate scaling of the bound on $h_{\mathrm{end}}$ as $H^{-1 / 3} T_{\max }^{4 / 3}$ can be understood in the following way. Being the Hubble rate large, the term $\left(a / H^{2}\right) \partial V / \partial h$ in eq. (4.1) can be neglected up to the moment when the second time derivative term or the first time derivative term become of the order of the potential term. This means that the Higgs field does not move much from its initial condition $h_{\text {end }}$ up to the moment when

$$
\frac{5}{2 a} \frac{d h}{d a} \sim \frac{a}{H^{2}} m_{T}^{2}(T) h=\frac{a}{H^{2}} T_{\max }^{2} a^{-3 / 4} h .
$$

This implies that the Higgs field starts moving away from $h_{\text {end }}$ when

$$
a \sim a_{*}=\left(\frac{45 H^{2}}{8 T_{\max }^{2}}\right)^{4 / 9} .
$$

Imposing that at this value of $a$ the finite temperature term in the potential dominates over the negative quartic term gives

$$
h_{\text {end }} \lesssim\left(\frac{8}{45}\right)^{1 / 6} \frac{H^{-1 / 3} T_{\max }^{4 / 3}}{\sqrt{|\lambda|}},
$$

which reproduces the right scaling shown in figure 9 . 

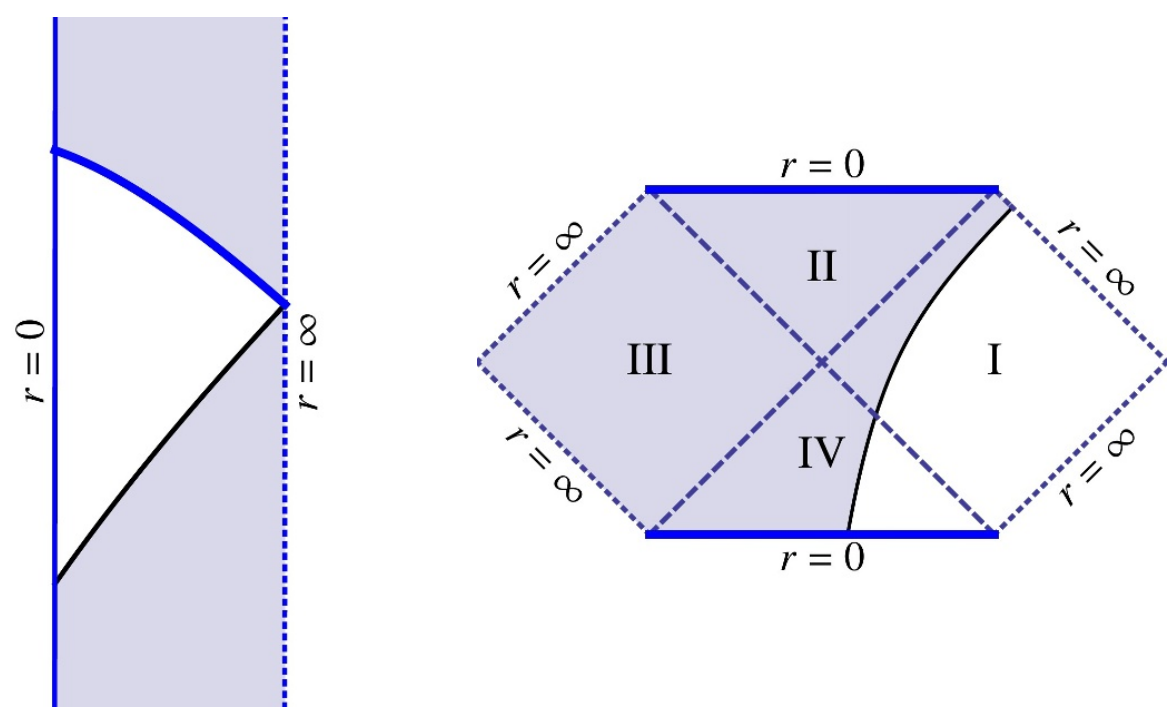

Figure 11. Penrose diagram describing an AdS bubble that expands in external Minkowski. Left: the wall trajectory corresponding to line $\mathrm{C}$ of figure 15, in AdS space. Right: the wall trajectory corresponding to line $\mathrm{C}$ of figure 15, in the Schwarzschild geometry.

In figure 9 we let $h_{\text {end }}$ and $H$ vary independently. However, the inflationary dynamics correlates the two variables, assigning a certain probability to $h_{\text {end }}$ for any given $H$. Although we have not used any relation between the two variables, in figure 9 we have indicated in red the region in which the field $h$ has overwhelming probability to slide towards large values and thus the corresponding parameters essentially cannot be the outcome of inflation.

Figure 10 shows the minimal value of $T_{\mathrm{RH}}$ for which the thermal corrections prevent the fall of $h$ down to its deep minimum. In other words, it shows how the limit on the Hubble constant $H$ corresponding to the orange area of figure 6 can be relaxed, depending on the reheating temperature $T_{\mathrm{RH}}$. For sufficiently large $T_{\mathrm{RH}}$, regions in which the Higgs field fluctuates around its instability scale $h_{\max }$ can be recovered by post-inflationary thermal effects. On the other hand, the thermal phase cannot save regions in which the Higgs fell down into its deep negative-energy minimum during inflation. In the next section we will show that most of these regions eventually expand and hence a viable cosmology require that no such regions are produced during inflation. This excludes the red area in figure 6 .

\subsection{Bubble evolution in Minkowski spacetime}

The discussion of bubble evolution in an external Minkowski spacetime is analogous to the de Sitter discussion of section 3.3. A first difference is that the effective potential of eq. (3.28), which dictates the evolution of the bubble, is simplified when we set $\ell_{\text {out }}=\infty$ (i.e. $\delta=0$ ). A second key difference is that the external Minkowski space has no causal horizons: if, after inflation ends, bubbles expand at the speed of light, they engulf the whole space.

An important task is to determine whether Higgs bubbles expand or shrink. The complete analysis is presented in appendix A, where all the possible wall trajectories are 
determined. In summary, there are two scenarios in which bubbles do not take over the whole space: either they start small enough so that they shrink, or they expand but remain hidden behind a black-hole horizon (a possibility that corresponds to $\Delta<0$ ). In the following we examine if either of these possibilities is realised for Higgs bubbles, making them benign.

We first consider bubbles with positive

$$
\Delta \equiv 1 / \ell_{\mathrm{in}}^{2}-(4 \pi G \sigma)^{2}>0
$$

which are bigger than their Schwarzschild radius and thereby can expand in the naive Newtonian way. This is the case depicted in the Penrose diagram in figure 11, to be compared with figure 7 for external dS. The black continuous curve denotes the trajectory of the wall: the bubble starts small and expands indefinitely within the asymptotically flat spacetime. The total space is constructed by patching the part of the diagram on the right of the wall with the part of the left diagram on the left of the wall. The shaded areas correspond to the parts that must be eliminated in order to join the remaining parts along the wall trajectory. From the point of view of an external observer, the bubble asymptotically expands at the speed of light and asymptotically reaches null infinity, filling all space.

Bubbles may also shrink because of their surface tension, if they are small enough ( $R<R_{\text {cr }}$ ), and start with a small wall velocity. The critical radius, separating the two types of evolution, can be computed from the potential of eq. (3.28), but the resulting expression is not very illuminating. We present the complete discussion in appendix A.6. The result can be simplified by assuming $\dot{R}=0$, and the Newtonian limit $\kappa \ll 1$ (such that $\Delta>0$ ). In this case, the critical radius is obtained by extremising the sum of the surface and volume energy $\left(4 \pi R^{2} \sigma-4 \pi R^{3} V_{\text {in }} / 3\right)$ with respect to $R$, thus finding $R_{\text {cr }}=2 \sigma / V_{\text {in }}$ and a bubble mass $M=16 \pi \sigma^{3} / 3 V_{\mathrm{in}}^{2}$. The exact result, valid even beyond the Newtonian approximation, is shown in figure 12. In the ultra-relativistic limit the critical radius becomes $R_{\text {cr }}=3 G M$, slightly larger than the Schwarzschild radius $2 G M$.

Figure 12 also shows the estimated $M(R)$ corresponding to Higgs bubbles for different values of $h_{\text {in }}$, the unknown Higgs value at its deep minimum. We use $V_{\text {in }} \sim|\lambda| h_{\text {in }}^{4}$ and estimate the surface tension from the Newtonian expression

$$
\sigma \approx \int d r\left[\frac{1}{2}\left(\frac{\partial h}{\partial r}\right)^{2}+V(h)-V\left(h_{\mathrm{in}}\right)\right] \sim \frac{h_{\mathrm{in}}^{2}}{\Delta r}+\Delta r|\lambda| h_{\mathrm{in}}^{4} \gtrsim \sqrt{|\lambda|} h_{\mathrm{in}}^{3}
$$

minimised for a bubble thickness $\Delta r \sim 1 /\left(h_{\text {in }} \sqrt{|\lambda|}\right)$.

The estimate for $\sigma$ is inserted in the full expression for $M$, eq. (A.11). The meaning of negative values of $M$ is discussed in the appendix, in sections A.4 and A.6: they correspond to bubbles for which the negative volume contribution to the total energy budget is dominant. For our present purposes, the conclusion is that inflationary fluctuations create a number of bubbles with a variety of values of $R$ and $\sigma$, including bubbles with $R>R_{\mathrm{cr}}$, which expand. Bubbles produced by inflationary fluctuations tend to appear with characteristic size $R \sim 1 / H=\ell_{\text {out }}$ and with negligible $\dot{R}$. If the Higgs value is near 


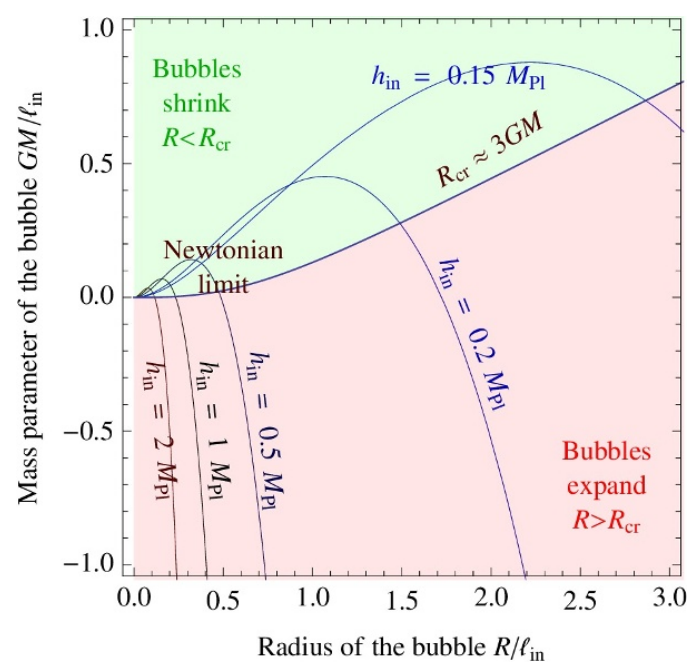

Figure 12. The boundary in the $(R, M)$ plane that separates expanding bubbles from shrinking bubbles. The curves are the estimated masses of Higgs bubbles, as functions of their radius, for different values of $h_{\text {in }}$, the unknown Higgs field value at the deep minimum.

the deep minimum of the potential, such bubbles are expected to have small or negative mass (because of the negative volume contribution to the energy) and, therefore, expand.

Bubbles with sufficiently large surface tension $\sigma$ have $\Delta<0$ and their expansion is energetically disfavoured from a Newtonian point of view. However, there exist expanding solutions for such bubbles within general relativity. They are discussed in appendix A (see figure 18). The crucial characteristic is that the expanding region is not accessible to an observer in the asymptotically flat space. In other words, an observer outside the bubble only sees a black-hole horizon, that protects him from its expansion. While such bubbles would be benign, $\Delta<0$ represents an extreme case: for the quartic Higgs potential, the surface tension gives a Planck-suppressed correction, such that

$$
\Delta \sim \frac{|\lambda|}{M_{\mathrm{Pl}}^{2}}\left[h_{\mathrm{in}}^{4}-\frac{h_{\mathrm{in}}^{6}}{M_{\mathrm{Pl}}^{2}}\right]
$$

is negative only when the deep minimum of the SM potential is super-Planckian, $h_{\mathrm{in}} \gtrsim M_{\mathrm{Pl}}$. It is then impossible to make firm predictions; strong gravitational effects may induce various dangerous effects. As long as the deep minimum is in the calculable sub-Planckian region $\left(h_{\mathrm{in}} \lesssim M_{\mathrm{Pl}}\right)$, bubbles have super-Planckian tension only for extreme Higgs field configurations, e.g. if the variation of $h$ between the two minima happens within a sub-Planckian length. Inflationary fluctuations tend to create bubbles with bigger thickness and smaller surface tension. Furthermore, even if the condition $\Delta<0$ were initially satisfied, the bubble would evolve towards a smoother configuration with smaller surface tension by reconfiguring the Higgs field profile.

Our study has been carried out within the thin-wall limit, because this is the only setup for which an analytical treatment is possible. As we pointed out above, the realistic situation is more likely to involve configurations with a smooth transition region from the interior AdS space to the false-vacuum exterior. For these, the fundamental dynamics 


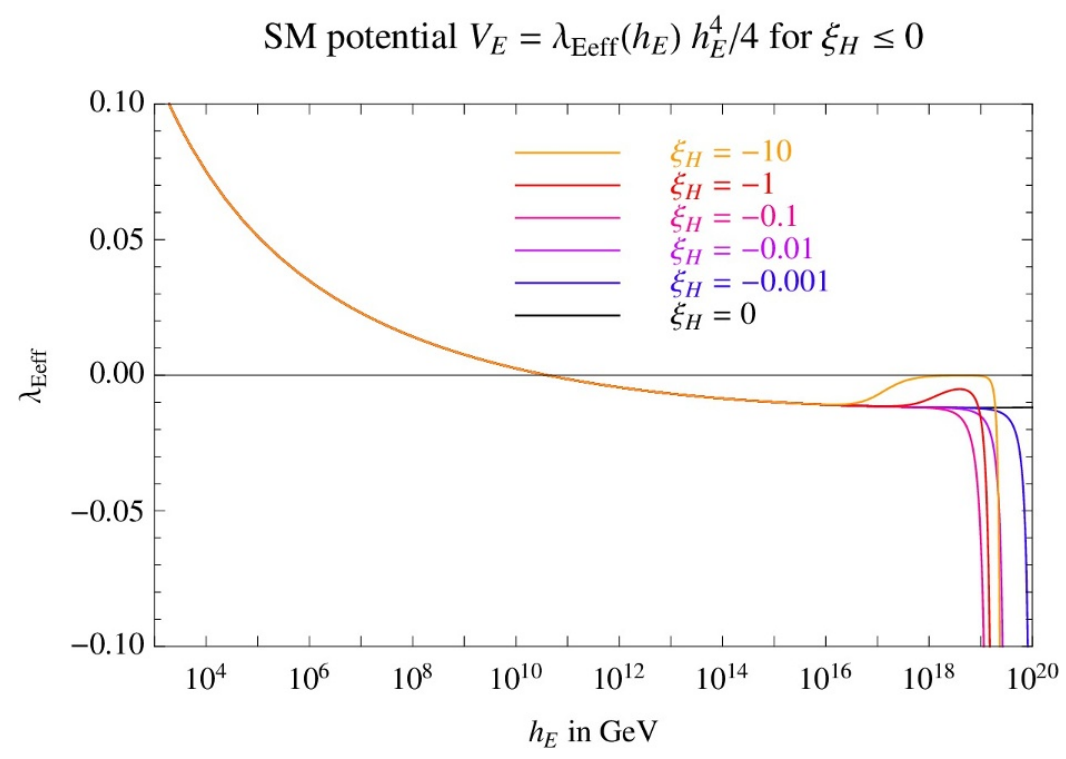

Figure 13. The SM Higgs potential $V_{E} \equiv \lambda_{E \text { eff }}\left(h_{E}\right) h_{E}^{4} / 4$ in the presence of a negative $\xi_{H}$ coupling, written in the Einstein frame in terms of the canonical Higgs field $h_{E}$.

is mainly determined through the interplay between the negative energy density in the interior and the positive contribution from the transition region. We expect that our analysis captures the essential features of the evolution of such configurations as well.

The important conclusion that we draw from our study is the following. No robust general-relativistic effect prevents large-field Higgs bubbles from expanding and engulfing all Minkowski space. As a result, a viable cosmology requires that no expanding bubbles are present in our past light-cone. In other words, the condition $p(h \rightarrow \infty)<e^{-3 N}$ for $N \approx 60$ $e$-folds must hold and the red region in figure 6 is excluded.

\subsection{The Higgs potential for $\xi_{H} \neq 0$ at zero temperature}

We conclude our study of the Higgs evolution after inflation with a remark concerning the non-minimal gravitational coupling $\xi_{H}$. As previously discussed, a coupling $\xi_{H}$ helps to stabilise the Higgs field during inflation. However, long after inflation, at zero temperature and $H \approx 0$, it could have an opposite effect in the classical potential, in presence of a Higgs instability generated by SM interactions $\left(\lambda_{\text {eff }}<0\right)$. In this setup an additional source of instability is generated by $\xi_{H}$ at Planckian values of the Higgs field, with no effect on our discussion of the Higgs dynamics during the inflationary and post-inflationary phase.

In order to investigate the phenomenon, we focus on the real component $h$ of the Higgs doublet $\Phi_{H}=(0, h / \sqrt{2})$ and perform a Weyl rescaling to the Einstein frame $g_{\mu \nu}^{E}=g_{\mu \nu} \times f$ with $f=1+\xi_{H} h^{2} / \bar{M}_{\mathrm{Pl}}^{2}$. Then, the Einstein-Hilbert term becomes canonical and the action is

$$
\begin{aligned}
\mathscr{L}_{E} & =\sqrt{\operatorname{det} g_{E}}\left[-\frac{\bar{M}_{\mathrm{Pl}}^{2}}{2} R_{E}+Z \frac{\left(\partial_{\mu} h\right)^{2}}{2}-V_{E}(h)\right]+\cdots \\
Z & =\frac{1}{f}+\bar{M}_{\mathrm{Pl}}^{2} \frac{3 f^{\prime 2}}{2 f^{2}}, \quad V_{E}(h)=\frac{V(h)}{f^{2}} .
\end{aligned}
$$


We are studying the theory long after inflation, and therefore $V(h)=\lambda_{\text {eff }}(h) h^{4} / 4{ }^{14}$ It is convenient to define a canonically normalised Einstein-frame Higgs field $h_{E}$ through the equation $d h_{E} / d h=\sqrt{Z}$, where $Z$ is given in eq. (4.21). The field $h_{E}$ is such that $h_{E} \simeq h$ for $h \ll \bar{M}_{\mathrm{Pl}}$ and $h_{E} \rightarrow \infty$ for $h \rightarrow \bar{M}_{\mathrm{Pl}} / \sqrt{-\xi_{H}}$ (hence $f \rightarrow 0$ ).

The Einstein-frame scalar potential becomes

$$
V_{E}\left(h_{E}\right)=\left.\frac{\lambda_{\mathrm{eff}}(h) h^{4}}{4\left(1+\xi_{H} h^{2} / \bar{M}_{\mathrm{Pl}}^{2}\right)^{2}}\right|_{h=h\left(h_{E}\right)} .
$$

In the limit of large $h_{E}$ (which corresponds to $h \rightarrow \bar{M}_{\mathrm{Pl}} / \sqrt{-\xi_{H}}$ ), the denominator in eq. (4.22) nearly vanishes, while $\lambda_{\text {eff }}$ is negative. In that regime of field configurations, we find $h\left(h_{E}\right)-\bar{M}_{\mathrm{Pl}} / \sqrt{-\xi_{H}} \propto \bar{M}_{\mathrm{Pl}} \exp \left(-\sqrt{2 / 3} h_{E} / \bar{M}_{\mathrm{Pl}}\right)$ and the potential becomes

$$
\frac{V_{E}\left(h_{E}\right)}{\bar{M}_{\mathrm{Pl}}^{4}} \propto \lambda_{\mathrm{eff}} \exp \left(\sqrt{\frac{8}{3}} \frac{h_{E}}{\bar{M}_{\mathrm{Pl}}}\right), \quad \text { for } h_{E} \rightarrow \infty .
$$

Here $\lambda_{\text {eff }}$ is evaluated at $h=\bar{M}_{\mathrm{Pl}} / \sqrt{-\xi_{H}}$ ) and is negative. The exponential behaviour in eq. (4.23) contributes to amplify the source of instability already present. The effect is shown in figure 13, where we plot the effective coupling $\lambda_{E \text { eff }}$, defined in analogy with previous effective quartic couplings by rewriting the potential in the Einstein frame as $V_{E}\left(h_{E}\right) \equiv \lambda_{E \text { eff }}\left(h_{E}\right) h_{E}^{4} / 4$. Negative values of $\xi_{H}$ appear to trigger a deep instability at Planckian field values $h \sim \bar{M}_{\mathrm{Pl}} / \sqrt{-\xi_{H}}$. However, for nonzero $\xi_{H}$, the ultraviolet cutoff of the SM is no longer $\bar{M}_{\mathrm{Pl}}$, but $\bar{M}_{\mathrm{Pl}} /\left|\xi_{H}\right|[57,58]$. Hence, the instability just described takes place above that cutoff, where one is losing control of the theory [59-61]. It has been pointed out that small primordial black holes can seed Higgs-vacuum decay and enhance its rate $[62,63]$. However, this process crucially depends on the number density of black holes at a given epoch.

\section{The quantum gravity prediction for the Higgs mass}

In this section we explore how a speculative conjecture that has been put forward in the context of quantum mechanical completions of gravity can lead to a sharp correlated prediction for the Higgs and top-quark masses. The intriguing result is that this prediction agrees quite well with the measured values of these masses. The reasoning is essentially based on two points.

1. The empirical observation that we live in an accelerating universe.

2. The theoretical conjecture that quantum gravity is ill-defined in de Sitter space $[64,65]$.

The difficulties with dS quantum gravity have been argued from various perspectives $[64,65]$. We summarise here in a very schematic way some of the arguments against

\footnotetext{
${ }^{14}$ During inflation $V$ contains an extra constant term $V_{\phi}$, which dominates the energy density, and $H^{2}=$ $V_{\phi} /\left(3 \bar{M}_{\mathrm{Pl}}^{2}\right)$ is the Hubble constant during inflation. By expanding $V_{E}$ at leading order in $h^{2} / \bar{M}_{\mathrm{Pl}}^{2}$ for $V_{\phi} \neq 0$, we recover the Higgs mass term considered in the previous sections, $m^{2}=-4 \xi_{H} V_{\phi} / \bar{M}_{\mathrm{Pl}}^{2}=-12 \xi_{H} H^{2}$. The higher order terms were not relevant for our previous discussion.
} 
a stable dS space, reviewed in [66], extending them in light of some more recent developments. There is no positive conserved energy in dS (and, as a consequence, there cannot be unbroken supersymmetry). There is no classical compactification of ten- or elevendimensional supergravity to dS space, and stable dS space cannot be obtained from any string or M-theory. Even in the general setting of quantum gravity, beyond the particular UV completion offered by string theory, other problems arise. It has been suggested that the quantum Hilbert space in dS is of finite dimension, limiting the variations of complex constructions. Given that the Gibbons-Hawking temperature sets a minimum temperature, the finite dimensionality of the Hilbert space sets a maximum time scale, the so-called recurrence time [65]. In particular, this leads to the problem of the so-called Boltzmann brains [67], and it has been suggested that its resolution calls for an unstable universe [67]. More generally, a rigorous definition of the Hilbert space in dS seems to be problematic [64]. In quantum gravity, it is difficult to define precisely local observables and one can rely only on asymptotic quantities, such as the $S$-matrix in Minkowski space and the boundary correlators in AdS. However, in dS, where asymptotic states fall behind the horizon, no such precisely defined observables seem to be present.

In addition to these problems that have been known for some time, it has been found in [68-70] that there is a sharp universal bound on how much reheating volume slow-roll inflation is capable to create without being eternal. This is given by $e^{S_{\mathrm{dS}}} / 2$, where $S_{\mathrm{dS}}$ is the entropy of the would be de Sitter space with Hubble rate evaluated at the time of reheating. Larger overall expansion is possible only by making space infinite. This generalises at the quantum level the bound on the duration of inflation found in [71], and determines the universality of such a bound under the number of fields involved, higher derivative corrections, number of space time dimensions and slow roll parameters. The same phenomenon, i.e. not being able to produce arbitrarily large finite volumes, is shared by false vacuum inflation. These results seem to suggest that there are bounds on the kind of global spacetime structures that local quantum field theory can generate. This is related to, and somewhat supporting, the argument against de Sitter space in nature that we follow here.

Though none of these arguments raise to the level of a proof of the inconsistency of dS space, they clearly give an idea of the conceptual difficulties that arise when considering quantum mechanics in dS space.

It could well be that the problems of asymptotic dS space are circumvented by Planckian dynamics, which can for example open channels for vacuum tunnelling to a 'landscape' of other minima with zero or negative vacuum energy. This is certainly a possibility. However, it is interesting to note that, even without any special hypothesis about the gravitational sector, the SM Higgs offers an easy way out to the problem. A solution is automatically found if the present dS space is only metastable.

As soon as the decay rate per unit space time volume $\Gamma$ of the false vacuum is non zero, the asymptotic space is not dS. True vacuum bubbles are nucleated and expand at the speed of light. There are two critical values of the decay rate [72], both valued around $H_{\Lambda}^{4}$, with $H_{\Lambda}$ being the Hubble rate of the would-be de Sitter region. 
If $\Gamma$ is larger than the largest critical point, bubbles percolate, fill the whole space, inflation ends globally, and the asymptotic spacetime is the one of the true vacuum (if this is AdS, the instability grows and leads to a singularity in about one Hubble time). The value of this critical point is $\Gamma_{2} / H_{\Lambda}^{4}=9 / 4 \pi \simeq 0.71$ [66].

The precise value of the second critical point is unknown, but is bounded to be in the range [72] $1.1 \times 10^{-6} \leq \Gamma_{1} / H_{\Lambda}^{4} \leq 0.24$. If the decay rate lies between these two critical points, then bubbles percolate and long chains of bubbles form, connecting arbitrarily distant point in the otherwise dS space. Finally, when the decay rate is even slower than the second critical point, then bubbles do not percolate, and the asymptotic spacetime is the one called 'false vacuum eternal inflation'. In this phase, there is an infinite amount of space that keeps inflating, but each single point decays at some time into the true vacuum through the nucleation of a bubble. ${ }^{15}$

On the other hand, there is no question that AdS and Minkowski are well-defined spaces from the point of view of quantum gravity, although we have a non-perturbative formulation of quantum gravity only for AdS. So, it is possible that quantum gravity allows for an eternally inflating false-vacuum space-time, but not for dS space. We will show that the opportunity of circumventing dS space through the Higgs field is given to nature only for a narrow range of Higgs boson masses. Interestingly, it seems that nature did not miss the opportunity because the measured Higgs boson mass lies exactly within this range.

Let us for a moment accept the quantum-gravity arguments against stable dS space, and let us assume that our universe, which we observe to be today in a dS phase, escapes the problems through a future decay of the Higgs vacuum. This implies the bound on the Higgs boson mass [1-3]

$$
\frac{M_{h}}{\mathrm{GeV}}<129.6+2.0\left(\frac{M_{t}}{\mathrm{GeV}}-173.34\right)-0.5\left(\frac{\alpha_{3}\left(M_{Z}\right)-0.1184}{0.0007}\right) \pm 0.3 .
$$

The vacuum decay rate induced by the SM Higgs instability, exponentially suppressed by the action of its bounce solution, $S \approx 8 \pi^{2} /(3|\lambda|) \sim 10^{3}$, is always faster, and typically much faster, than what is needed to avoid the $\mathrm{dS}$ problems. Indeed, for comparison, the de Sitter entropy is $S_{\mathrm{dS}} \sim \pi M_{\mathrm{Pl}}^{2} / H_{\Lambda}^{2} \sim 10^{120}$. In general, the action of any Coleman-de Luccia instanton out of a false vacuum with positive energy density is never larger than $S_{\mathrm{dS}}$, no matter how high we make the false vacuum barrier (see the discussion of [66] for a review). ${ }^{16}$ Therefore, when the Coleman-de Luccia instanton is present and can be reliably computed, the lifetime is bound by the Poincaré recurrence time of de Sitter space, of order $e^{S_{\mathrm{dS}}} / H$, up to logarithmic factors. It is expected that this lower bound on the decay rate is a property shared by all theories with a de Sitter false vacuum within field theory and perturbative gravity.

\footnotetext{
${ }^{15}$ Bubbles continuously form and collide an infinite number of times among each other but they do so in relatively smaller and smaller regions: there are points in the inflationary space for which the probability that they are connected by a stream of bubbles is zero. When bubbles collide in our past light cone, a very sharply defined disk shape is impressed in the CMB [73]. The optimal analysis to search for such a signal in the WMAP data has been recently performed, with no evidence found $[74,75]$.

${ }^{16}$ One can check this by taking the limit in which the wall energy density goes to infinity in eq. (3.16) of the paper by Coleman and de Luccia [25].
} 

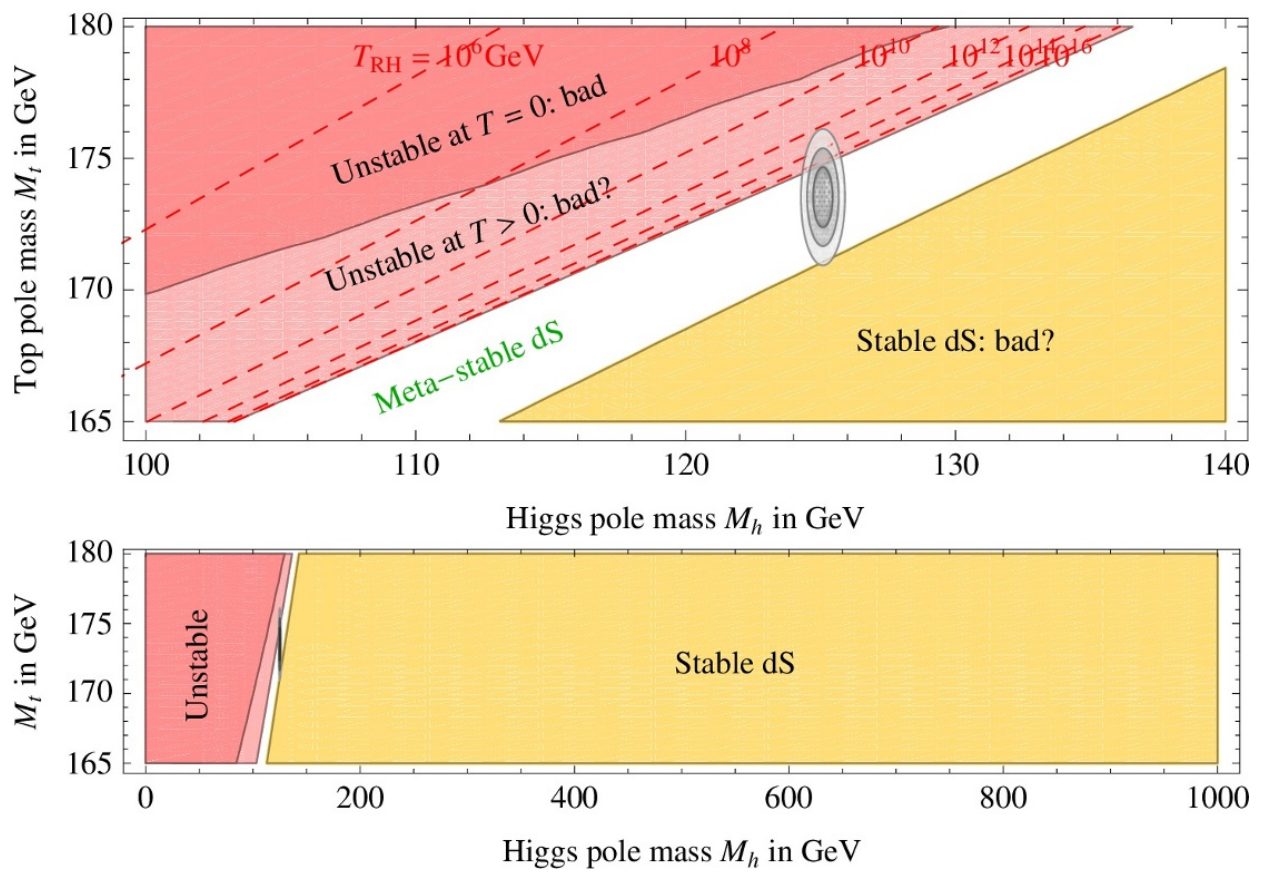

Figure 14. The allowed meta-stability window of the Higgs mass. The ellipse indicates the measured values of $M_{h}$ and $M_{t}$. The orange region is excluded by assuming that "stable dS" is unacceptable. The red region is excluded by vacuum decay at zero temperature. The pale-red region is excluded by the requirement that the universe must have been hot in the past (the dashed red curves show boundaries for different values of the reheating temperature). The bottom panel shows the same result in the full range of a-priori possible Higgs masses, in order to emphasise the smallness of the surviving meta-stability region.

There are also lower bounds on the Higgs mass. A first bound is obtained by requiring that the tunnelling rate away from the EW breaking state with small and positive cosmological constant is not faster than the age of the universe. In the current universe, the volume of our past light cone at the current time $T_{U}$ is

$$
\operatorname{Vol}_{4}\left(T_{U}\right)=0.08 H_{\Lambda}^{4}
$$

where $H_{\Lambda}^{2}=\Lambda^{4} / 3 \bar{M}_{\mathrm{Pl}}^{2}$ is the Hubble rate produced by the observed vacuum energy $\Lambda^{4}$. Imposing that the probability $p=e^{-\operatorname{Vol}_{4}\left(T_{U}\right) \Gamma}$ that our universe experienced vacuum decay in the past is small enough, implies an upper bound on the vacuum decay density rate $\Gamma$

$$
\Gamma<\frac{1}{\operatorname{Vol}_{4}\left(T_{U}\right)} \log \left(\frac{1}{p}\right)
$$

and, within the SM, a lower bound on the Higgs mass [1-3]

$$
\frac{M_{h}}{\mathrm{GeV}}>111+2.8\left(\frac{M_{t}}{\mathrm{GeV}}-173.34\right)-0.9\left(\frac{\alpha_{s}\left(M_{Z}\right)-0.1184}{0.0007}\right) \pm 1 .
$$

A stronger lower bound is obtained from the requirement that the universe underwent a hot phase. There are good reasons to believe that the universe has been very hot at 
an early epoch. Indeed, processes such as inflation and leptogenesis suggest that the primordial universe reached high temperatures. We have seen in section 4.2 how a large reheating temperature helps in forcing the Higgs to its weak scale meta-stable minimum. However, such high temperatures could have prematurely destabilised the Higgs metastable vacuum. The requirement that this did not happen implies

$$
\frac{M_{h}}{\mathrm{GeV}}>124.2-\frac{190}{\log _{10}^{2} \frac{T_{\mathrm{RH}}}{\mathrm{GeV}}}+2.0\left(\frac{M_{t}}{\mathrm{GeV}}-173.34\right)-0.6\left(\frac{\alpha_{s}\left(M_{Z}\right)-0.1184}{0.0007}\right) \pm 1 .
$$

Equations (5.1) and (5.5) define a fairly narrow range of possible Higgs masses (see figure 14). Loosely speaking, one might claim that quantum-gravity favours

$$
122 \mathrm{GeV}<M_{h}<129.4 \mathrm{GeV} \quad \text { for } M_{t}=173.34 \mathrm{GeV} .
$$

Given that the Higgs mass is now precisely measured, one can better use $M_{h}$ as input and predict the top mass in the range

$$
171 \mathrm{GeV}<M_{t}<175 \mathrm{GeV} .
$$

The coincidence that the Higgs and top masses are within the predicted range can be viewed as an indirect indication that nature took the opportunity offered by the Higgs to avoid the problem of an asymptotic dS space.

\section{Conclusions}

Assuming that the SM holds up to large energies, we studied under which conditions the cosmological evolution does not disrupt the electroweak vacuum, in spite of the presence of an instability of the SM effective Higgs potential $V(h)$ at field values $h>h_{\max }$.

As a preliminary step, in section 2 we clarified the gauge-dependence of the effective potential. The Nielsen identities show that the gauge-dependence of the effective action corresponds to different ways of parameterising the same physics in field space: the physical content of the effective action is gauge-independent. We have shown how this implies that the classical equation of motion (as well as the related Langevin and Fokker-Planck equations used later) are gauge-independent, because the gauge-dependence of the effective potential is compensated by the gauge-dependence of the kinetic term. Furthermore, we showed how, in the basis in which the kinetic term is canonical, the full effective potential becomes gauge-independent in the limit in which only the leading-log corrections are retained (which, for our purposes, is a very good approximation, see figure 1). For the present best-fit values of the SM parameters one has $h_{\max } \approx 5 \times 10^{10} \mathrm{GeV}$, but $h_{\text {max }}$ can vary by orders of magnitude if the top mass $M_{t}$ is varied within its uncertainty band.

Next, in section 3 we studied Higgs fluctuations during inflation. In our study, we also took into account the effect of a Higgs mass $m^{2}$ induced, during inflation, either by a mixed quartic coupling between the Higgs and the inflaton, or by a non-minimal Higgs coupling to gravity $\xi_{H}$. Not being radiatively stable, such a coupling is expected to be generally present and leads to $m^{2}=-12 \xi_{H} H^{2}$, where $H$ is the Hubble constant during inflation, 
given by $H \approx 8 \times 10^{13} \mathrm{GeV} \sqrt{r / 0.1}$. Present cosmological data constrain $r \lesssim 0.1$, but future measurements will have greater sensitivity.

If $m^{2}<9 H^{2} / 4$ the Higgs undergoes inflationary quantum fluctuations, which we computed via a Langevin equation that bypasses the need of imposing appropriate boundary conditions encountered in the Fokker-Planck equation used in previous works. We find that the parameter space in the plane $H / h_{\max }$ vs $\xi_{H}$ splits into 3 regions (see figure 6 ):

- 'green' region, where the Higgs remains below its instability scale at the end of inflation, and thus inflationary fluctuations do not destabilise the electroweak vacuum.

- 'orange' region, where the Higgs can probe field values above the instability scale $\left(|h|>h_{\max }\right)$, but quantum fluctuations dominate over classical evolution and prevent the Higgs from falling into its true AdS minimum; the ultimate fate of the Higgs is determined by post-inflationary dynamics.

- 'red' region, where the Higgs fluctuates above the instability scale and falls down into its true minimum, presumably ending inflation in that patch of space.

In section 4 we followed the evolution of the Higgs field through the reheating process, in order to assess the viability of parameters corresponding to the 'orange' region. Thermal effects can rescue the Higgs field, letting it slide towards the origin of the SM potential, if the reheating temperature after inflation $T_{\mathrm{RH}}$ is sufficiently large. We derived upper bounds on $H / h_{\max }$, for given $T_{\mathrm{RH}}$, as shown in figure 10 . The result is that thermal effects can easily make the 'orange' region cosmologically acceptable.

On the contrary, we found that the 'red' region is problematic. By approximating the large-field Higgs patches as spherical bubbles with small thickness, we could perform a general relativistic computation in order to determine whether such bubbles shrink or expand. The computation addresses several relevant counter-intuitive phenomena. While we identified mechanisms that can make some of the bubbles innocuous (small bubbles with low wall velocity shrink, bubbles with large tension expand hidden behind a blackhole horizon), we find that inflation produces Higgs 'bubbles' that expand, at least as long as they are in the computable sub-Planckian regime. During inflation these bubbles are not lethal, as they remain behind a de Sitter horizon and are diluted by space expansion. However, after inflation they keep on growing at the speed of light, eventually swallowing all space. Therefore, we must require that inflationary fluctuations do not produce any of these regions in our past light-cone.

This leads us to our final result: the 'red' region of figure 6 is excluded. If $\left|\xi_{H}\right|<$ 0.01 one needs a Hubble constant smaller than $0.045 h_{\max }$. This constraint gets weaker (stronger) for negative (positive) $\xi_{H}$ : e.g. $H<10^{4} h_{\max }$ for $\xi_{H} \approx-0.03$. A small negative $\xi_{H}$ however leads to a new, super-Planckian instability of the SM potential in the Einstein frame, see figure 13. In a similar way, a direct coupling of the inflaton to the Higgs could also relax the limits on $H$ but, contrary to the case of $\xi_{H}$, it does not lead to any instabilities at large field values.

Finally, in section 5 we explore a new speculative idea. Assuming that the present acceleration of the universe is due to a small cosmological constant, and accepting the 
conjecture that quantum gravity is ill-defined in a de Sitter space, we argue that vacuum decay is a necessary way out for the universe. We show that vacuum decay triggered by the Higgs instability is fast enough to resolve this conceptual problem.

Basically the SM phase diagram in the $\left(M_{t}, M_{h}\right)$ plane is reinterpreted: the instability region remains 'bad', the stability region becomes 'bad', and the only 'good' region is the narrow meta-stability strip of parameter space. As discussed in section 4.2 a large enough reheating temperature may play an important role in the universe, and the requirement that thermal effects do not induce an excessively fast vacuum decay provides a further restriction in the Higgs and top masses, as shown in figure 14. One could view this restriction as a remarkably precise post-diction for the Higgs or top masses.

\section{Acknowledgments}

We thank M. Garny and T. Konstandin for very useful discussions and Gino Isidori and Joan Elias-Miró for participating in the early stages of this work. J.R.E. thanks CERN for hospitality and partial financial support. This work was supported by the ESF grant MTT8. The work of J.R.E. has been supported by the Spanish Ministry MEC under grants FPA2013-44773-P, FPA2012-32828; by the Generalitat grant 2014-SGR-1450 and by the Severo Ochoa excellence program of MINECO (grant SO-2012-0234). L.S. is supported by the DOE Early Career Award DE-FG02-12ER41854, by the National Science Foundation under PHY-1068380, and by the European Commission under the ERC Advanced Grant BSMOXFORD 228169. The work of N.T. has been co-financed by the European Union (European Social Fund ESF) and Greek national funds through the Operational Program "Education and Lifelong Learning" of the National Strategic Reference Framework (NSRF) - Research Funding Program: "THALIS. Investing in the society of knowledge through the European Social Fund".

\section{A Evolution of bubbles}

In this appendix we study the evolution of a region of true vacuum with negative vacuum energy density, which lies within the false-vacuum asymptotically flat or de Sitter space. The basic question is whether this region, which we call a bubble (assuming spherical symmetry), expands or contracts. For an outside observer, the presence of the bubble has a gravitational effect equivalent to the presence of a central mass. As a result, the exterior metric is of the Schwarzschild or Schwarzschild-de Sitter (SdS) type. We study an idealised configuration with constant vacuum energy density in the interior and exterior of the bubble, as well as constant surface tension. The study of a realistic bubble, corresponding to a space-time dependent Higgs configuration, is not possible analytically. However, we believe that our treatment captures the main aspects of the problem, determined essentially by the difference in the local energy density of the Higgs field on either side of the bubble wall.

We employ the thin-wall approximation and parameterise the wall and the inner and outer space as described in section 3.3. The interior of the bubble is assumed to be a part of anti-de Sitter spacetime, described by the metric (3.23). The exterior of the bubble is 
described by the metric (3.24). The case $V_{\text {out }} \neq 0$ corresponds to the SdS spacetime, while the case $V_{\text {out }}=0$ to an exterior Schwarzschild metric. The metric on the wall is given by eq. (3.25).

\section{A.1 Matching the geometries}

The metric must be continuous over the whole space. This means that

$$
\begin{gathered}
f_{\text {in }}(R) \dot{\eta}=\epsilon_{1}\left(\dot{R}^{2}+f_{\text {in }}(R)\right)^{1 / 2}, \\
f_{\text {out }}(R) \dot{t}=\epsilon_{2}\left(\dot{R}^{2}+f_{\text {out }}(R)\right)^{1 / 2}
\end{gathered}
$$

where $\epsilon_{1}= \pm 1, \epsilon_{2}= \pm 1$ are possible sign choices and a dot denotes a derivative with respect to $\tau$. Since $f_{\text {in }} \geq 1$, the value of $\epsilon_{1}$ determines the relative flow of the two timelike coordinates $\eta$ and $\tau$. It is natural to make the choice $\epsilon_{1}=1$, which is also the only consistent choice (see below). We consider only this value in the following. The relation between $t$ and $\tau$ is more complicated because $f_{\text {out }}$ can be negative. We follow the convention of [76], according to which the flow of proper time is such that future-directed world lines correspond to a growing Kruskal-Szekeres coordinate $V$ (so that $\dot{V}>0$ ).

The matching of the two regions can be done following [76]. The four-velocity of a point on the wall is $U_{\text {in }}^{\mu}=(\dot{\eta}, \dot{R}, \overrightarrow{0})$ and $U_{\text {out }}^{\mu}=(\dot{t}, \dot{R}, \overrightarrow{0})$ in each of the frames. A spacelike vector $\xi^{\mu}$ perpendicular to the wall must be orthogonal to $U^{\mu}$. In order to determine it uniquely, we have to specify whether it points towards the interior or the exterior. For spaces with horizons, such as the exterior space, we adopt the convention of [76]. We assume that $\xi^{\mu}$ points towards increasing values of the Kruskal-Szekeres coordinate $U$. We also assume that the exterior lies on the 'right' of the wall in the Penrose diagram. For the AdS space in the interior, we assume that $\xi^{\mu}$ points towards increasing values of the global coordinate $r$. With these conventions $\xi^{\mu}$ points from the interior towards the exterior. It is given by

$$
\begin{aligned}
\xi_{\text {in }}^{\mu} & =\left(\frac{\dot{R}}{f_{\text {in }}}, f_{\text {in }} \dot{\eta}, \overrightarrow{0}\right)=\left(\frac{\dot{R}}{f_{\text {in }}},\left(f_{\text {in }}+\dot{R}^{2}\right)^{1 / 2}, \overrightarrow{0}\right) \\
\xi_{\text {out }}^{\mu} & =\left(\frac{\dot{R}}{f_{\text {out }}}, f_{\text {out }} \dot{t}, \overrightarrow{0}\right)=\left(\frac{\dot{R}}{f_{\text {out }}}, \epsilon_{2}\left(f_{\text {out }}+\dot{R}^{2}\right)^{1 / 2}, \overrightarrow{0}\right),
\end{aligned}
$$

in each of the frames. It has been normalized to -1 .

The junction conditions connect the discontinuity in the extrinsic curvature to the surface tension:

$$
\left(K_{\text {out }}\right)_{j}^{i}-\left(K_{\text {in }}\right)^{i}{ }_{j}=-4 \pi \sigma G \delta^{i}{ }_{j}
$$

We match the $\theta \theta$ component of the extrinsic curvature (the other components give equivalent relations), which is $K_{\theta \theta}=\xi^{\mu} \partial_{\mu} r^{2} / 2$. Evaluated on either side of the wall, it is given by

$$
\begin{aligned}
\left(K_{\text {in }}\right)_{\theta \theta} & =\left(f_{\text {in }}+\dot{R}^{2}\right)^{1 / 2} R \equiv \beta_{\text {in }} R, \\
\left(K_{\text {out }}\right)_{\theta \theta} & =\epsilon_{2}\left(f_{\text {out }}+\dot{R}^{2}\right)^{1 / 2} R \equiv \beta_{\text {out }} R .
\end{aligned}
$$


Thereby, the $\theta \theta$ matching condition is

$$
\epsilon_{2}\left(f_{\text {out }}+\dot{R}^{2}\right)^{1 / 2}-\left(f_{\text {in }}+\dot{R}^{2}\right)^{1 / 2}=\beta_{\text {out }}-\beta_{\text {in }}=-4 \pi G \sigma R .
$$

\section{A.2 Bubbles in asymptotically flat spacetime}

We consider first the case $V_{\text {out }}=0$, which corresponds to an exterior Schwarzschild metric. We shall discuss later the case $V_{\text {out }} \neq 0$, corresponding to a SdS spacetime.

The square of eq. (A.8) can be put in the form:

$$
2 G M=\left(\kappa^{2}-\frac{1}{\ell_{\mathrm{in}}^{2}}\right) R^{3}+2 \epsilon_{2} \kappa R^{2}\left(1-\frac{2 G M}{R}+\dot{R}^{2}\right)^{1 / 2} .
$$

For large $R$ and non-relativistic wall velocity, the last parenthesis becomes equal to 1 . The resulting expression indicates that the mass $M$ of a large-radius bubble is dominated by a volume contribution proportional to $\kappa^{2}-1 / \ell_{\mathrm{in}}^{2}$. The total volume effect can be negative or positive, depending on the value of

$$
\epsilon \equiv \operatorname{sign}\left(\frac{1}{\ell_{\mathrm{in}}^{2}}-\kappa^{2}\right) .
$$

As a result, it is possible for the total mass $M$ to become negative.

Solving eq. (A.8) for $M$ one finds a result that, for $\epsilon_{2}=1$, has a simple Newtonian interpretation:

$$
2 G M=-\left(\frac{1}{\ell_{\mathrm{in}}^{2}}+\kappa^{2}\right) R^{3}+2 \kappa R^{2}\left(1+\frac{R^{2}}{\ell_{\mathrm{in}}^{2}}+\dot{R}^{2}\right)^{1 / 2},
$$

with $\kappa \equiv 4 \pi G \sigma$. For small $R$, the mass $M$ attributed to the bubble of AdS by an outside observer contains a volume term proportional to $-1 / \ell_{\mathrm{in}}^{2}-\kappa^{2}$. The contribution $-1 / \ell_{\mathrm{in}}^{2}$ corresponds to the vacuum energy density, while $-\kappa^{2}$ reproduces correctly the gravitational self-energy of the wall. The second term in eq. (A.11) can be expanded for small $R$ and $\dot{R}$. One recovers the surface energy of the bubble, with nonrelativistic correction, and the surface-volume binding energy [72]. The leading term for small $R$ is the positive surface energy $\sim \kappa R^{2}$, which indicates that small bubbles tend to collapse in order to minimise their energy. The case $\epsilon_{2}=-1$ does not lead to solutions with a simple Newtonian interpretation, even though it contains acceptable configurations for the global geometry.

By squaring eq. (A.8) a second time, we can express the 'kinetic energy' $\dot{R}^{2}$ in terms of a conserved 'energy' $E$ and an effective 'potential energy'. We express the result as the equation for the one-dimensional motion of a particle in a 'potential' $V$

$$
\left(\frac{d \tilde{R}}{d \tilde{\tau}}\right)^{2}+V(\tilde{R})=E
$$

where

$$
V(\tilde{R})=-\left(\frac{\epsilon_{m}+\epsilon \tilde{R}^{3}}{\tilde{R}^{2}}\right)^{2}-\epsilon_{m} \frac{\gamma^{2}}{\tilde{R}}, \quad E=-\frac{\kappa^{2}}{G^{2} M^{2} \rho^{4}}
$$


and

$$
\epsilon_{m}=\operatorname{sign}(M) .
$$

The dimensionless 'coordinate' variable $\tilde{R}$ and the 'time' variable $\tilde{\tau}$ are defined as

$$
\tilde{R}=\rho R, \quad \tilde{\tau}=\frac{2 \kappa}{\gamma^{2}} \tau .
$$

The parameter $\rho$, defined as

$$
\rho^{3}=\frac{1}{2 G|M|}\left|\frac{1}{\ell_{\mathrm{in}}^{2}}-\kappa^{2}\right|,
$$

sets a characteristic inverse length-scale, while $\gamma$ parameterises the surface-energy term in $V$ :

$$
\gamma=\frac{2 \kappa}{\left|\ell_{\mathrm{in}}^{-2}-\kappa^{2}\right|^{1 / 2}}, \quad \text { i.e. } \quad \kappa^{2}=\frac{1}{\ell_{\mathrm{in}}^{2}} \frac{\gamma^{2}}{\gamma^{2}+4 \epsilon} .
$$

The form of the solutions of eq. (A.12) can be revealed more easily through the following observations:

- The sign $\epsilon_{2}$ disappeared when performing the second squaring, so that eq. (A.12) describes the solutions of eq. (A.8) with both values of $\epsilon_{2}$. We can rewrite eq. (A.8) in terms of the new parameters as

$$
\beta_{\text {in }}=\beta_{\text {out }}+4 \pi G \sigma R=\frac{G|M| \rho^{2}}{\kappa} \frac{1}{\tilde{R}^{2}}\left(\epsilon_{m}+\epsilon \tilde{R}^{3}+\frac{\gamma^{2}}{2} \tilde{R}^{3}\right),
$$

where we have used eq. (A.11). For positive-mass bubbles $\left(\epsilon_{m}=1\right)$ we have $\beta_{\text {in }}>0$. This is obvious for $\epsilon=1$. It also holds for $\epsilon=-1$, because $\gamma^{2}>4$ in this case. We conclude that the only consistent value for $\epsilon_{1}$ for positive-mass bubbles is $\epsilon_{1}=1$ (the value we assumed).

- We can also write

$$
\beta_{\text {out }}=\frac{G|M| \rho^{2}}{\kappa} \frac{1}{\tilde{R}^{2}}\left(\epsilon_{m}+\epsilon \tilde{R}^{3}\right),
$$

from which it is apparent that, for positive-mass bubbles, $\beta_{\text {out }}$ is positive and $\epsilon_{2}=1$ for $\epsilon=1$, while $\beta_{\text {out }}$ changes sign at $\tilde{R}=1$ for $\epsilon=-1$.

- For negative-mass bubbles $\left(M<0\right.$ or $\left.\epsilon_{m}=-1\right)$ the variable $t$ is always timelike. The value of $\epsilon_{2}$ determines the relative flow of $t$ and $\tau$. It is natural to make the choice $\epsilon_{2}=1$ in this case. The possibility $\epsilon_{2}=-1$ does not lead to a physical solution, as we discuss in subsection A.4.

- It is apparent from eqs. (A.13), (A.16) that, for fixed $\ell_{\text {in }}$ and $\kappa$, the total energy $E$ is a function of $M$. As a result, the nature of the various solutions of eq. (A.12) is directly related to the mass of the bubble.

- The 'potential' is maximal at $\tilde{R}=\tilde{R}_{\text {max }}$, given by

$$
2 \tilde{R}_{\max }^{3}=\epsilon_{m}\left(\epsilon+\frac{\gamma^{2}}{2}\right)+\sqrt{\left(\epsilon+\frac{\gamma^{2}}{2}\right)^{2}+8} .
$$



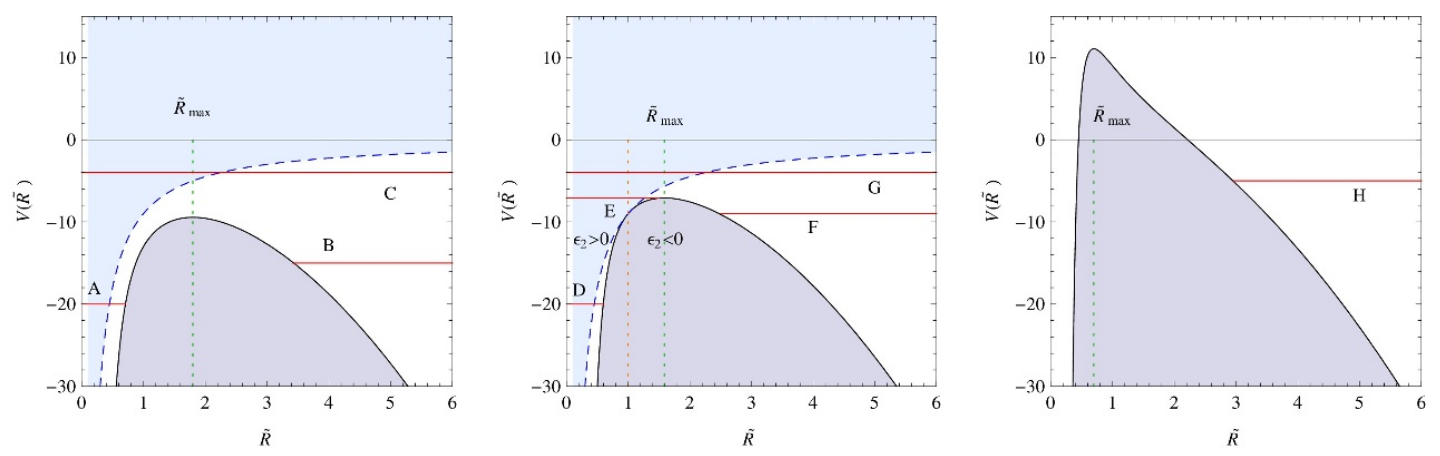

Figure 15. The 'potential' of eq. (A.13) for $\gamma=3$ and $\epsilon=1, \epsilon_{m}=1$ (left), for $\epsilon=-1, \epsilon_{m}=1$ (middle), and for $\epsilon=1, \epsilon_{m}=-1$ (right).

The value of the 'potential' at its maximum is

$$
V\left(\tilde{R}_{\max }\right)=-3 \frac{\tilde{R}_{\max }^{6}-1}{\tilde{R}_{\max }^{4}} .
$$

For positive-mass bubbles $\left(\epsilon_{m}=1\right)$ we have $\tilde{R}_{\max }>1$.

- The Schwarzschild radius of a bubble with positive mass $M$ is $r_{H}=2 G M$, which, in terms of the variable $\tilde{R}$, becomes

$$
E=-\frac{\gamma^{2}}{\tilde{R}_{H}}
$$

This relation determines the location of the horizon on a solution of eq. (A.12) with given E. Making use of the definition (A.13) of the 'potential', we can write

$$
E=V\left(\tilde{R}_{H}\right)+\left(\frac{1+\epsilon \tilde{R}_{H}^{3}}{\tilde{R}_{H}^{2}}\right)^{2}
$$

For $\epsilon=-1$ the curve $-\gamma^{2} / \tilde{R}_{H}$, depicting the location of the horizon, is tangent to the curve $V=V(\tilde{R})$ at $\tilde{R}=1$. For $\epsilon=1$ the curve for the horizon is always located above the curve for the 'potential'.

The above features are depicted graphically in figure 15 . The solid black curve depicts the 'potential' $V(\tilde{R})$, which has a maximum at $\tilde{R}=\tilde{R}_{\max }$. The dashed blue curve indicates the location of the horizon. For $\epsilon=1$ (i.e. $1 / \ell_{\text {in }}^{2}>\kappa^{2}$ ) the curve for the horizon is always located above the "potential'. For $\epsilon=-1$ (i.e. $1 / \ell_{\mathrm{in}}^{2}<\kappa^{2}$ ) the curve for the horizon is tangent to the 'potential' at $\tilde{R}=1$. The function $\beta_{\text {out }}$ changes sign at this point. In the centre plot we have separated with a red vertical dashed line the regions in which $\epsilon$ (and $\beta_{\text {out }}$ ) has opposite signs.

The various types of trajectories can be deduced from these plots. We plot a few lines with constant $E$ that stop when $E=V$ : at this point $\dot{R}=0$ and the motion of the wall is reversed. There are various types of trajectories for which the bubble expands indefinitely. If $\epsilon=-1$, such evolution can be obtained only for $\epsilon_{2}=-1$. 

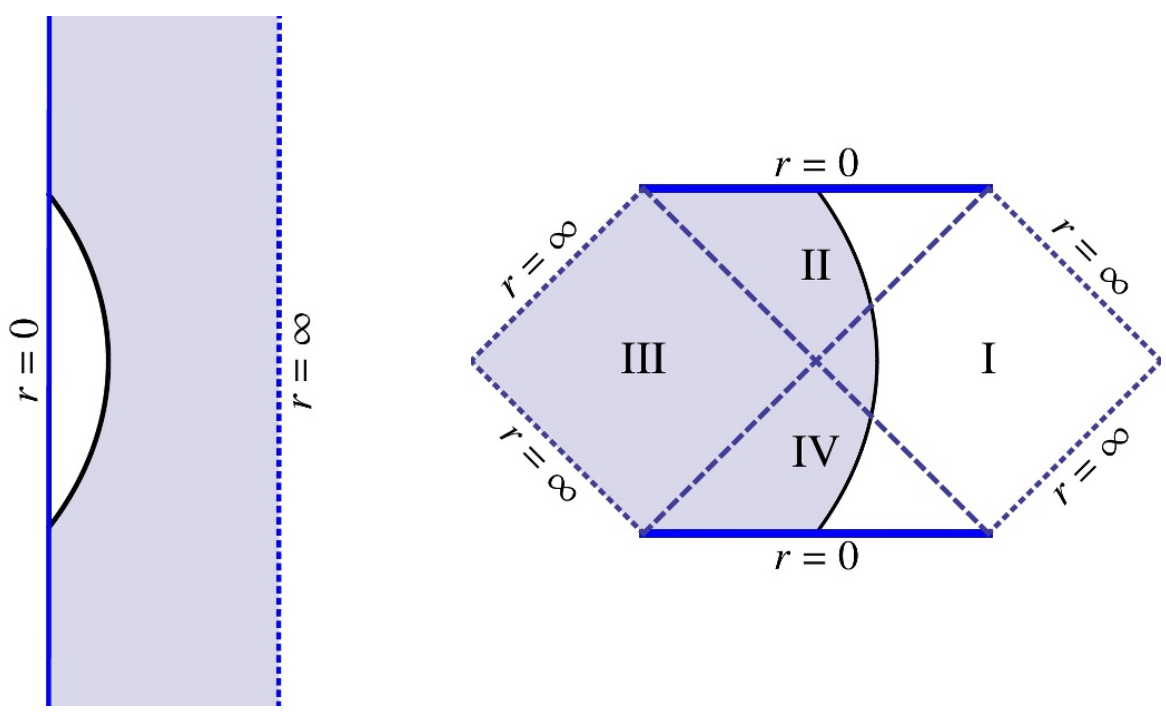

Figure 16. Small bubbles with small initial wall velocity do not expand. Left: the wall trajectory corresponding to line A of figure 15, in AdS space. Right: the wall trajectory corresponding to line A of figure 15, in the Schwarzschild geometry.

\section{A.3 Evolution of positive-mass bubbles}

We consider first the case $M>0$, or, equivalently, $\epsilon_{m}=1$. The evolution of the wall is best depicted using Penrose diagrams. The diagrams for the most characteristic types of wall evolution are presented in figures 16, 11, 18. Each figure contains a pair of diagrams. In each pair, the left diagram depicts AdS space, which has the simple structure of a cylinder, with the line marked $r=0$ corresponding to its centre and the line $r=\infty$ to conformal infinity. The right diagram represents the complete Schwarzschild geometry, which includes two singularities, marked $r=0$, and the corresponding horizons. The black continuous curve in each diagram denotes the trajectory of the wall. Thick black lines denote singularities, dashed lines horizons and dotted lines denote conformal infinities. The total space is constructed by patching the part of the left diagram on the left of the wall with the part of the right diagram on the right of the wall. The shaded areas correspond to the parts that must be eliminated in order to join the remaining parts along the wall trajectory.

The crucial relation for the fate of space is between the gravitational self-energy of the wall $\kappa^{2}$ and the vacuum energy $-1 / \ell_{\mathrm{in}}^{2}$. Naively, one expects that, if $1 / \ell_{\mathrm{in}}^{2}>\kappa^{2}$ (i.e. $\epsilon=1$ ), large bubbles will grow indefinitely because the system gains energy in the process. In the opposite case with $1 / \ell_{\mathrm{in}}^{2}<\kappa^{2}$ (i.e. $\epsilon=-1$ ), the bubbles will shrink for similar energetic reasons. These simple expectations, which are based on Newtonian intuition, are only partly fulfilled in the complete analysis. More complicated scenarios are realised as well.

\section{Case $\epsilon=1$ :}

- Small bubbles with small initial wall velocity do not expand: line A of figure 15 describes the evolution of a bubble whose volume energy receives its largest contribution 

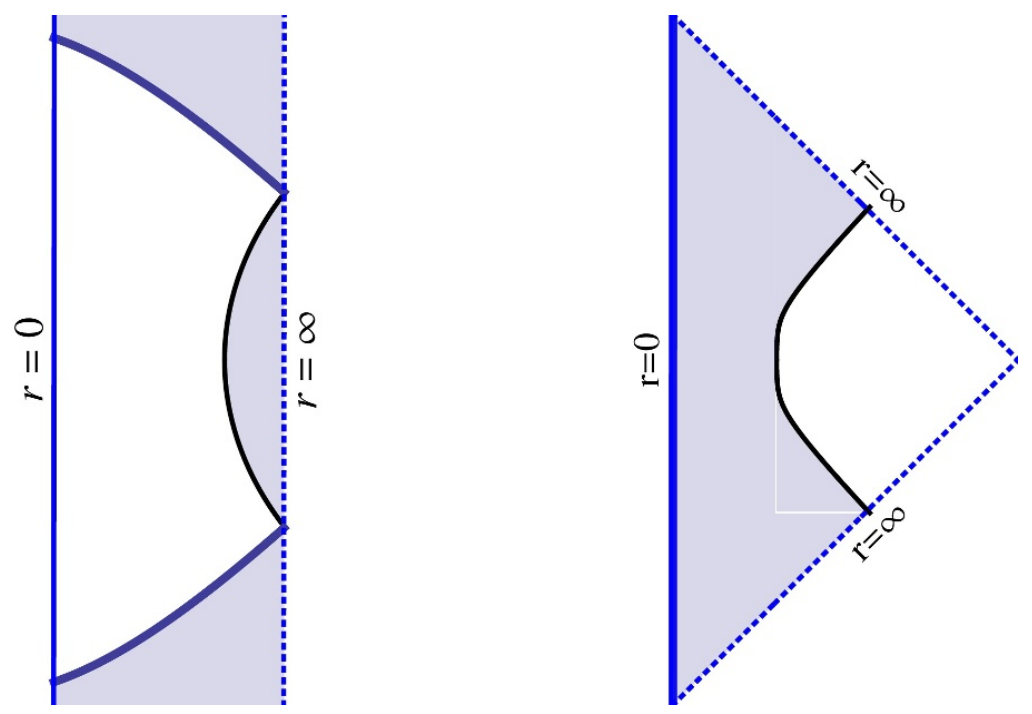

Figure 17. The evolution of negative-mass bubbles. Left: the wall trajectory corresponding to line $\mathrm{H}$ of figure 15, in AdS space. Right: the wall trajectory corresponding to line $\mathrm{H}$ of figure 15, in the negative-mass Schwarzschild geometry.

from the negative vacuum energy density $\left(1 / \ell_{\mathrm{in}}^{2}>\kappa^{2}\right)$. However, the surface contribution to the energy, arising from the wall tension, is the dominant factor and tends to make the bubble shrink. The bubble has small initial wall velocity, which prevents it from evolving to a size sufficiently large for the volume contribution to the energy to dominate. As a result the surface tension wins: the bubble reaches a maximum size and subsequently collapses falling within its own horizon.

The space corresponding to this solution is depicted in figure 16. It results from eliminating the shaded areas in each of the two Penrose diagrams and patching the remaining parts along the wall trajectory.

- Small bubbles with large initial wall velocity expand: line $\mathrm{C}$ of figure 15 corresponds to a bubble with similar characteristics as in the previous case, but with much larger wall 'kinetic energy'. This is is apparent by the fact that the total energy is less negative. We can consider a bubble that starts very small (with almost vanishing $r$ or $\tilde{R}$ ). Even though the surface contribution to the 'potential energy' dominates, the initial velocity is sufficiently large for the bubble to expand. Eventually the bubble develops a size for which the volume contribution to the 'potential energy' becomes dominant over the surface contribution. From this point on, the bubble expands indefinitely, with its wall approaching asymptotically the speed of light.

The corresponding evolution of space is depicted in figure 11. After a finite time $\eta$ the wall reaches the boundary of AdS space. As the AdS boundary is timelike, there is a Cauchy horizon, beyond which the spacetime cannot be determined without additional boundary conditions. It is expected that, within the full theory beyond the thin-wall limit, a spacelike singularity develops before the Cauchy horizon [52]. This 

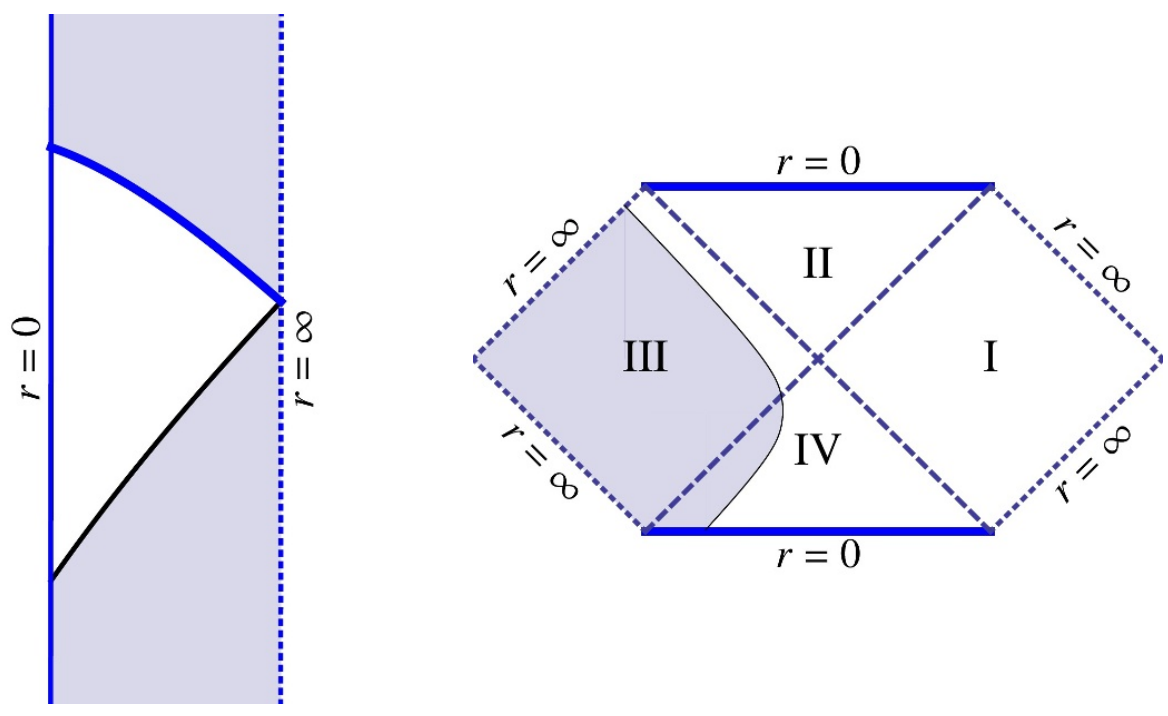

Figure 18. Large bubbles with $1 / \ell_{\mathrm{in}}^{2}<\kappa^{2}$ expand behind the horizon. Left: the wall trajectory corresponding to Line $\mathrm{G}$ of figure 15, in AdS space. Right: The wall trajectory corresponding to Line $\mathrm{G}$ of figure 15, in the Schwarzschild geometry.

is depicted by a thick blue line in the left diagram of figure 11. From a mathematical point of view, the solution also describes the reverse process.

- Large bubbles expand: line B of figure 15 describes the evolution of a bubble so large that its surface tension is irrelevant. The bubble starts with infinite radius, shrinks to finite size and then re-expands. There are two singularities in the Penrose diagram of AdS space, starting from the points at which the wall trajectory reaches the boundary [52]. The whole trajectory lies with the region I of the Schwarzschild geometry.

\section{Case $\epsilon=-1$ :}

- Small bubbles with small initial wall velocity do not expand: line D of figure 15 describes evolution very similar to that for line A. The contribution from the surface tension dominates the 'potential energy', while the 'kinetic energy' is small. The bubble expands up to a certain size, and subsequently recollapses. The space is described by Penrose diagrams very similar to those of figure 16 .

Line $\mathrm{E}$ of figure 15 describes a similar scenario, but now the extrinsic curvature $\beta_{\text {out }}$ (or, equivalently $\epsilon_{2}$ ) changes sign during the evolution. This implies that the wall trajectory crosses regions IV, III and II of the Schwarzschild geometry instead of the regions IV, I, II (see figure 16).

- Small bubbles with large initial wall velocity expand behind the horizon: as we have seen already, the case $\epsilon=-1$ may lead to evolution that cannot be deduced through a purely Newtonian approach. For $1 / \ell_{\mathrm{in}}^{2}<\kappa^{2}$ the wall self-energy dominates the 
negative vacuum energy, so that the growth of the bubble seems energetically unfavourable. The Newtonian intuition suggests that such bubbles cannot expand. However, there is a relativistic solution described by line $\mathrm{G}$ of figure 15 . The corresponding space evolution is depicted in figure 18. The crucial difference with respect to the case $\epsilon=1$, depicted in figure 11, is that the wall trajectory is located within the regions IV and III, instead of the regions IV and I of the Schwarzschild space-time: in simpler words the bubble expands inside its Schwarzschild radius. The extrinsic curvature $\beta_{\text {out }} R$ of eq. (A.7) changes sign along the trajectory $\mathrm{G}$ of the wall, while it stays positive for the trajectory $\mathrm{C}$, as can be seen in figure 15 [76]. Asymptotic regions of flat space-time survive: the growth of the AdS region and its singularity are hidden behind the horizon and do not affect an observer located in region I. ${ }^{17}$

- Large bubbles expand behind the horizon: line F of figure 15 describes a large bubble that initially shrinks, reaches a minimal size and subsequently expands. The whole evolution lies entirely within the region III of the Schwarzschild space-time and is hidden behind a horizon for an observer located in region I.

\section{A.4 Evolution of negative-mass bubbles}

We next turn to the solutions with negative mass $M$, or, equivalently, $\epsilon_{m}=-1$. The metric (3.24), with $V_{\text {out }}=0$, has a naked timelike singularity at $r=0$ in this case. However, this metric is relevant only for the bubble exterior, while the interior is described by the AdS metric (3.23). As long as the bubble expands and the wall moves to increasing values of $R$, the global geometry is free of singularities.

The form of the 'potential' for $1 / \ell^{2}>\kappa^{2}, M<0$, depicted in the right plot of figure 15 , allows for such a solution. For $\epsilon=1, \epsilon_{m}=-1$, the 'potential' has a positive maximal value. On the other hand, the 'energy' $E$ of eq. (A.13) is always negative. This allows for only one possible type of solutions, the one corresponding to line $\mathrm{H}$ of figure 15 . It represents a bubble that starts with infinite radius, shrinks to a finite value of $R$, and subsequently re-expands. Its mass is negative, because the radius is always sufficiently large for the negative volume contribution to the energy content to be dominant. The Penrose diagram for this solution is depicted in figure 17. There are two singularities in the Penrose diagram of AdS space, starting from the points at which the wall trajectory reaches the boundary. The Schwarzschild metric with negative mass has a naked singularity at $r=0$, depicted by the vertical solid line in the right plot of figure 17. However, this singularity is irrelevant for our problem because it is eliminated when the white areas of the two plots are joined along the wall trajectory.

It must be pointed out that it is not possible to construct negative-mass solutions corresponding to horizontal lines extending from $\tilde{R}=0$ to the 'potential' in figure 15 . As can be seen from eq. (A.19) such lines would require $\beta_{\text {out }}<0$, or, equivalently $\epsilon_{2}=-1$. As we have already remarked, this choice would require the timelike coordinates $\tau$ and $t$ to increase in opposite directions. For positive mass, the Schwarzschild geometry has sufficient

\footnotetext{
${ }^{17}$ A pictorial representation of an analogous situation for a dS bubble is given in figure 13 of [76], in which case the AdS singularity is absent.
} 


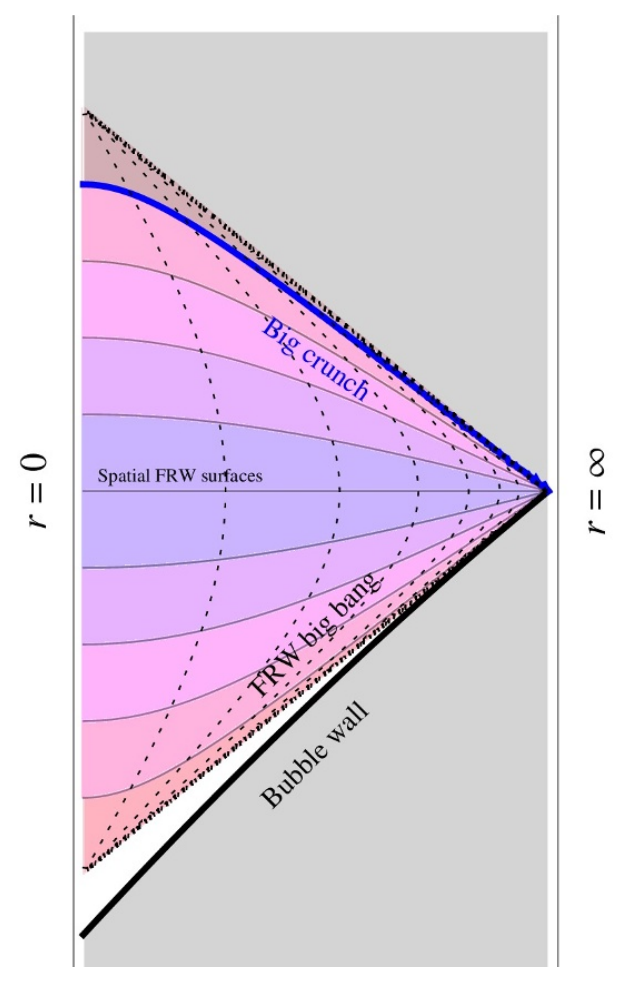

Figure 19. The AdS interior of the bubble in conformal coordinates, showing the crunch and (in color) a patch in FRW coordinates.

structure to permit solutions with both signs of $\beta_{\text {out }}$, such as the one corresponding to line $\mathrm{G}$ of figure 15, which is depicted in figure 18. However, for negative mass, the Penrose diagram cannot be extended beyond that depicted in figure 17, unless completely disjointed regions are introduced. For this reason, the only meaningful solution is the one of figure 17.

\section{A.5 The AdS 'crunch'}

As we have seen, the particular structure of AdS implies that the evolution of the bubble must lead to a singularity. This is apparent in figure 11: the form of spacetime, after the finite time $\eta$ at which the wall reaches the timelike boundary, cannot be determined without additional boundary conditions. It is expected that, for a physical system that realises an approximation of the idealised bubble evolution that we consider, a spacelike singularity must develop in the interior of the bubble [52].

For the problem at hand, the nature of this singularity can be understood through the picture of the AdS 'crunch' presented in [25]. As shown there, a part of AdS space can be viewed as an open Friedmann-Robertson-Walker (FRW) universe with negative energy density. The coordinate change [77]

$$
\begin{aligned}
r & =\ell_{\text {in }} \sin \frac{\hat{t}}{\ell_{\text {in }}} \sinh \psi \\
\cos \frac{\hat{t}}{\ell_{\text {in }}} & =\left(1+\frac{r^{2}}{\ell_{\text {in }}^{2}}\right)^{1 / 2} \cos \frac{\eta}{\ell_{\text {in }}}
\end{aligned}
$$


puts the AdS metric (3.23) in the form

$$
d s^{2}=-d \hat{t}^{2}+\ell_{\mathrm{in}}^{2} \sin ^{2} \frac{\hat{t}}{\ell_{\mathrm{in}}}\left(d \psi^{2}+\sinh ^{2} \psi d \Omega_{2}^{2}\right) .
$$

This metric describes an homogeneous FRW universe that is born with a big 'bang' at $\hat{t}=0$ and collapses in a big 'crunch' at $\hat{t}=\ell_{\text {in }} \pi$. The coordinates $\hat{t}, \psi$ do not cover the whole AdS space, but only a triangular patch of its Penrose diagram. This is bounded by the dot-dashed null lines in figure 19. Even though these lines represent only a coordinate singularity in the idealized picture of a pure AdS bubble interior, the big 'crunch' becomes a true physical singularity in the presence of a fluctuating Higgs field, as argued in [25]. From continuity it is apparent that the Higgs fluctuations become very large in the neighbourhood of the null line.

The connection of the AdS 'crunch' to the bubble evolution can be obtained by establishing the relative position of the FRW 'triangle' and the wall trajectory on the Penrose diagram. As the FRW observer views a homogeneous universe, the AdS patch to which he has access must be located sufficiently deep inside the bubble for the Higgs field to have a constant value. At late times, the wall moves with approximately the speed of light. It is expected that the wall trajectory and the lower side of the FRW 'triangle' will converge asymptotically as the AdS boundary is approached, as depicted in figure 19. The singularity developing below the Cauchy horizon appears on a spacelike curve emanating from the point at which the wall reaches the AdS boundary. The homogeneity of space viewed by the FRW observer indicates that this singularity should correspond to a constant- $\hat{t}$ surface. In figure 19 we depict the slicing of the FRW 'triangle' with such surfaces. The thick solid line represents the possible location of the 'crunch', very close to the upper side of the FRW 'triangle'.

The most important consequence of the above picture is that the 'crunch' never reaches the bubble wall. This is apparent in figure 19, as the black solid line, representing the wall trajectory, and the blue solid line, representing the 'crunch', never cross. They seem to merge on the AdS boundary. However, this is an illusion created by the Penrose diagram. The bubble wall lies always slightly outside the FRW 'triangle', as its speed never becomes exactly equal to that of light.

A final observation relevant for the asymptotic wall expansion concerns the corresponding time scales in the various frames. Let us consider a very large bubble with $\epsilon_{1}=\epsilon_{2}=1$, see eqs. (A.1)-(A.2), expanding almost at the speed of light, such that $R \gg \ell_{\text {in }}, 2 G M$ and $\dot{R} \gg 1$. The evolution of the wall in terms of the three time coordinates of the systems (3.25), (3.23), (3.24) is given by

$$
R=R_{0} e^{c_{1} \tau}=\frac{R_{0}}{1-c_{2} \eta}=R_{0}+t
$$

with $c_{1} \simeq 1 /\left(2 \ell_{\mathrm{in}}^{2} \kappa\right), c_{2} \simeq R_{0} / \ell_{\mathrm{in}}^{2}$ for $1 / \ell_{\mathrm{in}}^{2} \gg \kappa^{2}$. It is apparent that the wall reaches the AdS boundary within a finite amount of the time coordinate $\eta$, while it requires an infinite amount of time $\tau$ or $t$. In particular, an observer in the asymptotically flat spacetime infinitely far from the bubble is reached by the wall only after an infinite amount of time $t$. 

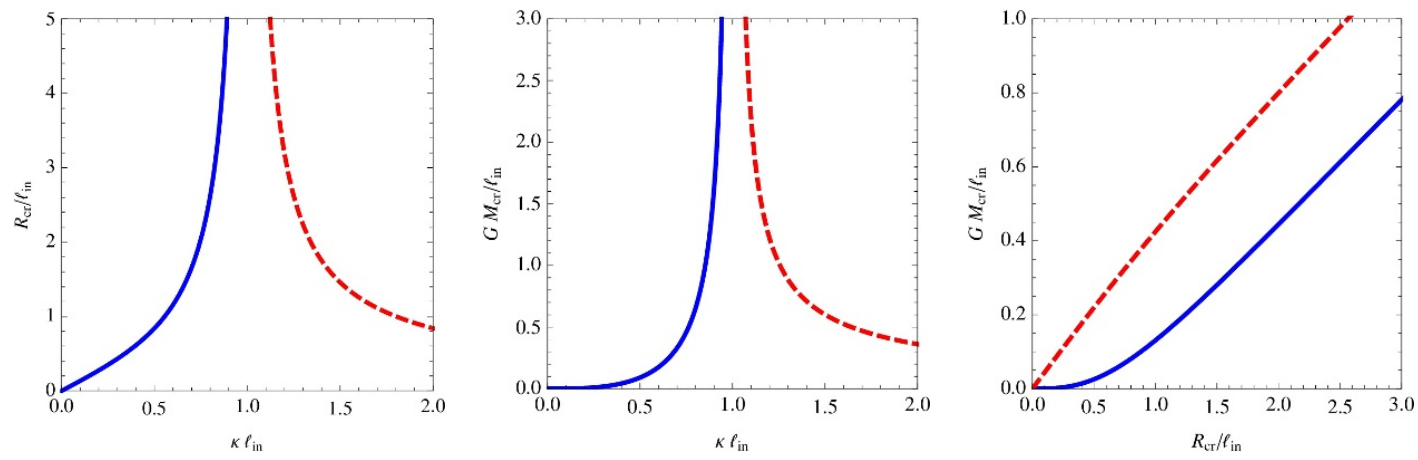

Figure 20. The radius and mass of critical bubbles as a function of $\kappa \ell_{\text {in }}$ for $\Delta>0$ (blue curves) and $\Delta<0$ (red dashed).

\section{A.6 Critical bubbles}

For given $\ell_{\text {in }}$ and $\kappa$, corresponding to given interior vacuum energy and surface tension, there is a critical bubble radius $R_{\mathrm{cr}}$. Bubbles that start with negligible wall velocity and $R>R_{\mathrm{cr}}$ follow trajectories with increasing $R$, while bubbles that start with $R<R_{\mathrm{cr}}$ have diminishing $R$ and eventually collapse to a black hole. The critical radius corresponds to the maximum of the potential of figure 15 . One can imagine a horizontal line, tangent to the top of the potential. The right part of the line is the limiting case of lines starting on the potential and describing expanding bubbles, while its left part is the limiting case of similar lines describing collapsing bubbles. As the 'energy' $E$ is negative, while the potential has a maximum with a positive value for $\epsilon_{m}=-1$ (see the third plot of figure 15), it is obvious that there are no critical bubbles with negative mass.

The maximum of the potential and its value at this point are given by eqs. (A.20)(A.21). The value of the 'energy' can be obtained from eq. (A.12) with $d \tilde{R} / d \tilde{\tau}=0$ and $\tilde{R}=\tilde{R}_{\text {max }}$. Expressions for $R_{\mathrm{cr}}$ and the corresponding mass $M_{\mathrm{cr}}$ can then be obtained by combining eqs. (A.13)-(A.17). As these expressions are not very illuminating we do not present them explicitly. The quantities $R_{\mathrm{cr}} / \ell_{\mathrm{in}}, G M_{\mathrm{cr}} / \ell_{\mathrm{in}}$ are functions of the dimensionless combination $\kappa \ell_{\mathrm{in}}$. In figure 20 we present the functions $R_{\mathrm{cr}}\left(\kappa \ell_{\mathrm{in}}\right) / \ell_{\mathrm{in}}, G M_{\mathrm{cr}}\left(\kappa \ell_{\mathrm{in}}\right) / \ell_{\mathrm{in}}$ and $\left[G M_{\mathrm{cr}} / \ell_{\mathrm{in}}\right]\left(R_{\mathrm{cr}} / \ell_{\mathrm{in}}\right)$.

The critical bubbles have certain characteristics:

- Their radius is always larger than the Schwarzschild radius. This can be deduced from figure 15, in which it is apparent that the location of the maximum of the potential is always outside the horizon.

- There are two branches of critical bubbles, corresponding to $1 / \ell_{\mathrm{in}}^{2}>\kappa^{2}$ or $\epsilon=1$ (solid lines), and $1 / \ell_{\mathrm{in}}^{2}<\kappa^{2}$ or $\epsilon=-1$ (dashed lines).

- The radius diverges for $\kappa \rightarrow 1 / \ell_{\text {in }}$, as the effective energy density in the interior of the bubble vanishes in this limit.

- The branch with $\epsilon=1$ reproduces correctly for $\kappa \rightarrow 0$ the Newtonian limit of nonrelativistic bubbles with $R_{\mathrm{cr}}=4 \ell_{\mathrm{in}}^{2} \kappa / 3$ and $G M_{\mathrm{cr}}=16 \ell_{\mathrm{in}}^{4} \kappa^{3} / 27$. 
- The branch with $\epsilon=-1$ is not visible to an observer located in region I of the Penrose diagram.

- For given vacuum-energy scale $\ell_{\mathrm{in}}$ and critical-bubble radius $R_{\mathrm{cr}}$, the bubbles with $\epsilon=-1$ are more massive than the ones with $\epsilon=1$. (Note that the two types of bubbles also have different surface tension $\kappa$.)

The most interesting solutions are those that describe bubbles visible to an observer in the asymptotically flat region. These are bubbles for which a Newtonian limit exists within their parameter range. Their mass-to-radius relation is depicted in the third plot of figure 20. The critical bubbles correspond to the solid line. The parameter range above this line corresponds to collapsing bubbles, while the range below to expanding bubbles. Expanding bubbles can have negative mass, so their parameter range includes the region below the positive $R$-axis.

\section{A.7 Bubbles in asymptotically de Sitter spacetime}

The evolution of an AdS bubble within an asymptotically dS spacetime can be analysed in complete analogy to the previous discussion for an asymptotically flat spacetime. The metric of eq. (3.24) now contains the function $f_{\text {out }}(r)=1-r^{2} / \ell_{\text {out }}^{2}-2 G M / r$. There are two horizons, corresponding to the zeros of $f_{\text {out }}(r)$. The Penrose diagram of the Schwarzschildde Sitter (SdS) spacetime is depicted in the right part of figure 7. It is a combination of the diagrams for the Schwarzschild and dS spacetimes [27]. Thick blue lines denote curvature singularities, the dashed lines horizons and the dotted lines conformal infinities. The two thin vertical lines at the ends of the diagram indicate that the pattern is repeated indefinitely on either side.

The matching across the domain wall, located at $R(\tau)$, proceeds as before. We do not analyse the many possible cases, as the analysis is a straightforward generalisation of the discussion in the previous subsections. We focus instead on the novel aspects of the SdS case. For a positive mass $M$, the motion of the wall is again determined by eq. (A.12), with $\tilde{R}=\rho R$. However, the 'potential' now has the form

$$
V(\tilde{R})=-\left(\frac{1+\epsilon \tilde{R}^{3}}{\tilde{R}^{2}}\right)^{2}-\frac{\gamma^{2}}{\tilde{R}}-\delta^{2} \tilde{R}^{2},
$$

with

$$
\rho^{3}=\frac{1}{2 G M}|\Delta|, \quad \epsilon \equiv \operatorname{sign} \Delta, \quad \gamma=\frac{2 \kappa}{\sqrt{|\Delta|}}, \quad \delta^{2}=\frac{4 \kappa^{2}}{\ell_{\mathrm{out}}^{2} \Delta^{2}},
$$

where

$$
\Delta=\frac{1}{\ell_{\mathrm{in}}^{2}}+\frac{1}{\ell_{\mathrm{out}}^{2}}-\kappa^{2} .
$$

The form of the 'potential' is very similar to that in figure 15 . The horizon corresponds to a value $\tilde{R}_{H}$ such that

$$
E=-\frac{\gamma^{2}}{\tilde{R}_{H}}-\delta^{2} \tilde{R}_{H}^{2}
$$




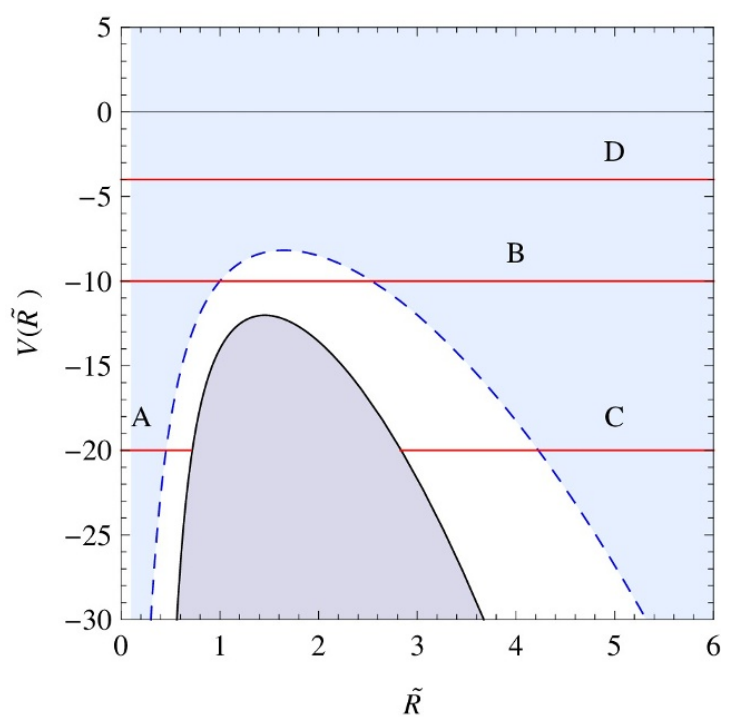

Figure 21. The 'potential' of eq. (A.27) with $\gamma=3, \delta=1, \epsilon=1$, for AdS bubbles in Schwarzschildde Sitter space.

It is again determined by eq. (A.23), but now has a different shape. In figure 21 we depict the 'potential' (solid black line) and the horizon (dashed blue line) for $\gamma=3, \delta=1, \epsilon=1$.

The various trajectories correspond to solutions of constant $E=-\kappa^{2} /\left(G^{2} M^{2} \rho^{4}\right)$, as depicted in figure 21. The two types of behaviour, characterised by $\epsilon= \pm 1$, correspond now to the gravitational self-energy $\kappa^{2}$ of the bubble being smaller or larger than the total difference in energy density $1 / \ell_{\mathrm{in}}^{2}+1 / \ell_{\text {out }}^{2}$ between the dS and AdS spacetimes. We do not analyse the form of all the possible trajectories, as they are similar to those discussed earlier. In figure 21 we have depicted a few characteristic cases for $\epsilon=1$.

Line A corresponds to a bubble that starts below the inner horizon, crosses it, reaches a maximal radius and collapses falling again behind the horizon.

Line $\mathrm{C}$ corresponds to a large bubble that stars with infinite radius, moves within the outer horizon, reaches a minimal radius and then re-expands moving again outside the outer horizon. One may consider also the scenario in which the bubble is spontaneously created with vanishing wall velocity at a certain radius and expands, with the wall moving outside the outer horizon. In this scenario, line $\mathrm{C}$ is covered only once.

Line B corresponds to a bubble that starts with a very small radius and expands indefinitely, with its wall crossing the inner and outer horizons successively. Its speed asymptotically approaches the speed of light. The form of the wall trajectory on the Penrose diagram is depicted in figure 7. The total space is constructed by patching the white regions of the two plots in figure 7 .

Line D corresponds to the evolution of a bubble that does not cross any horizons. The reason is that 'energies' that approach zero correspond to increasing values of the mass parameter $M$. For sufficiently large $M$, the metric function $f_{\text {out }}(r)$ does not vanish at any $r$, but stays always negative. The space has a naked spacelike singularity at $r=0$. However, this part of spacetime is eliminated and replaced by the interior of the AdS bubble. 
There are many other possibilities for $\epsilon=-1$ or for negative bubble mass. These can be analysed in complete analogy to the trajectories depicted in the second and third plot of figure 15. They correspond to collapsing bubbles or bubbles expanding behind horizons, which are not visible to an observer located in the asymptotic de Sitter space.

The crucial question pertinent to the scenario of Higgs fluctuations during inflation is whether the expanding AdS bubbles can completely eliminate the surrounding dS space and thus terminate inflation. It is apparent from figure 7 that asymptotically the wall trajectory reaches spacelike infinity. The wall location separates two spacelike regions: one of them is replaced by the interior of the AdS bubble, while the other remains part of an external dS spacetime. Asymptotically the total spacetime contains large AdS bubbles within large dS regions. This scenario is in contrast to the case of asymptotically flat spacetime, in which the wall asymptotically reaches null infinity and the whole space is engulfed by the AdS bubbles. In other words, the inflationary growth guarantees that, even when the size of the AdS regions grows with the speed of light, the external regions grow even faster, so that they survive at late times.

Open Access. This article is distributed under the terms of the Creative Commons Attribution License (CC-BY 4.0), which permits any use, distribution and reproduction in any medium, provided the original author(s) and source are credited.

\section{References}

[1] D. Buttazzo, G. Degrassi, P.P. Giardino, G.F. Giudice, F. Sala, A. Salvio et al., Investigating the near-criticality of the Higgs boson, JHEP 12 (2013) 089 [arXiv:1307.3536] [INSPIRE].

[2] G. Degrassi et al., Higgs mass and vacuum stability in the Standard Model at NNLO, JHEP 08 (2012) 098 [arXiv: 1205.6497] [INSPIRE].

[3] J. Elias-Miro, J.R. Espinosa, G.F. Giudice, G. Isidori, A. Riotto and A. Strumia, Higgs mass implications on the stability of the electroweak vacuum, Phys. Lett. B 709 (2012) 222 [arXiv: 1112.3022] [INSPIRE].

[4] M. Sher, Electroweak Higgs potentials and vacuum stability, Phys. Rept. 179 (1989) 273 [INSPIRE].

[5] P.B. Arnold, Can the electroweak vacuum be unstable?, Phys. Rev. D 40 (1989) 613 [INSPIRE].

[6] G. Altarelli and G. Isidori, Lower limit on the Higgs mass in the standard model: an update, Phys. Lett. B 337 (1994) 141 [INSPIRE].

[7] J.A. Casas, J.R. Espinosa and M. Quirós, Standard model stability bounds for new physics within LHC reach, Phys. Lett. B 382 (1996) 374 [hep-ph/9603227] [INSPIRE].

[8] T. Hambye and K. Riesselmann, Matching conditions and Higgs mass upper bounds revisited, Phys. Rev. D 55 (1997) 7255 [hep-ph/9610272] [InSPIRE].

[9] G. Isidori, G. Ridolfi and A. Strumia, On the metastability of the standard model vacuum, Nucl. Phys. B 609 (2001) 387 [hep-ph/0104016] [InSPIRE].

[10] G. Isidori, V.S. Rychkov, A. Strumia and N. Tetradis, Gravitational corrections to standard model vacuum decay, Phys. Rev. D 77 (2008) 025034 [arXiv: 0712.0242] [INSPIRE]. 
[11] J. Ellis, J.R. Espinosa, G.F. Giudice, A. Hoecker and A. Riotto, The probable fate of the standard model, Phys. Lett. B 679 (2009) 369 [arXiv:0906.0954] [INSPIRE].

[12] F. Bezrukov, M. Yu. Kalmykov, B.A. Kniehl and M. Shaposhnikov, Higgs boson mass and new physics, JHEP 10 (2012) 140 [arXiv:1205.2893] [INSPIRE].

[13] S. Alekhin, A. Djouadi and S. Moch, The top quark and Higgs boson masses and the stability of the electroweak vacuum, Phys. Lett. B 716 (2012) 214 [arXiv:1207.0980] [INSPIRE].

[14] J.R. Espinosa, G.F. Giudice and A. Riotto, Cosmological implications of the Higgs mass measurement, JCAP 05 (2008) 002 [arXiv:0710.2484] [INSPIRE].

[15] A. Kobakhidze and A. Spencer-Smith, Electroweak vacuum (in)stability in an inflationary universe, Phys. Lett. B 722 (2013) 130 [arXiv:1301.2846] [INSPIRE].

[16] K. Enqvist, T. Meriniemi and S. Nurmi, Generation of the Higgs condensate and its decay after inflation, JCAP 10 (2013) 057 [arXiv: 1306.4511] [INSPIRE].

[17] M. Fairbairn and R. Hogan, Electroweak vacuum stability in light of BICEP2, Phys. Rev. Lett. 112 (2014) 201801 [arXiv: 1403.6786] [INSPIRE].

[18] K. Enqvist, T. Meriniemi and S. Nurmi, Higgs dynamics during inflation, JCAP 07 (2014) 025 [arXiv: 1404.3699] [INSPIRE].

[19] A. Kobakhidze and A. Spencer-Smith, The Higgs vacuum is unstable, arXiv:1404.4709 [INSPIRE].

[20] M. Herranen, T. Markkanen, S. Nurmi and A. Rajantie, Spacetime curvature and the Higgs stability during inflation, Phys. Rev. Lett. 113 (2014) 211102 [arXiv:1407.3141] [INSPIRE].

[21] K. Kamada, Inflationary cosmology and the standard model Higgs with a small Hubble induced mass, Phys. Lett. B 742 (2015) 126 [arXiv:1409.5078] [INSPIRE].

[22] A. Shkerin and S. Sibiryakov, On stability of electroweak vacuum during inflation, Phys. Lett. B 746 (2015) 257 [arXiv: 1503.02586] [INSPIRE].

[23] A. Hook, J. Kearney, B. Shakya and K.M. Zurek, Probable or improbable universe? Correlating electroweak vacuum instability with the scale of inflation, JHEP 01 (2015) 061 [arXiv: 1404.5953] [INSPIRE].

[24] J. Kearney, H. Yoo and K.M. Zurek, Is a Higgs vacuum instability fatal for high-scale inflation?, Phys. Rev. D 91 (2015) 123537 [arXiv:1503.05193] [INSPIRE].

[25] S.R. Coleman and F. De Luccia, Gravitational effects on and of vacuum decay, Phys. Rev. D 21 (1980) 3305 [INSPIRE].

[26] S.W. Hawking and I.G. Moss, Supercooled phase transitions in the very early universe, Phys. Lett. B 110 (1982) 35 [INSPIRE].

[27] G.W. Gibbons and S.W. Hawking, Cosmological event horizons, thermodynamics and particle creation, Phys. Rev. D 15 (1977) 2738 [INSPIRE].

[28] A.A. Starobinsky, Cosmological models with the intermediate de Sitter stage: theory and observational consequences, in Fundamental interactions, MGPI Press, Moscow Russia (1984).

[29] A.A. Starobinsky, Stochastic de sitter (inflationary) stage in the early universe, in Current topics in field theory, quantum gravity and strings, in Current topics in field theory, quantum gravity and strings, H.J. de Vega and N. Sanchez eds., Springer (1986). 
[30] A.D. Linde, Eternally existing selfreproducing chaotic inflationary universe, Phys. Lett. B 175 (1986) 395 [INSPIRE].

[31] A.D. Linde, Hard art of the universe creation (stochastic approach to tunneling and baby universe formation), Nucl. Phys. B 372 (1992) 421 [hep-th/9110037] [INSPIRE].

[32] L. Di Luzio and L. Mihaila, On the gauge dependence of the standard model vacuum instability scale, JHEP 06 (2014) 079 [arXiv:1404.7450] [INSPIRE].

[33] A. Andreassen, W. Frost and M.D. Schwartz, Consistent use of effective potentials, Phys. Rev. D 91 (2015) 016009 [arXiv: 1408.0287] [INSPIRE].

[34] A. Andreassen, W. Frost and M.D. Schwartz, Consistent use of the standard model effective potential, Phys. Rev. Lett. 113 (2014) 241801 [arXiv:1408.0292] [INSPIRE].

[35] N.K. Nielsen, Removing the gauge parameter dependence of the effective potential by a field redefinition, Phys. Rev. D 90 (2014) 036008 [arXiv: 1406.0788] [INSPIRE].

[36] S.H.H. Tye and Y. Vtorov-Karevsky, Effective action of spontaneously broken gauge theories, Int. J. Mod. Phys. A 13 (1998) 95 [hep-th/9601176] [INSPIRE].

[37] W. Buchmüller, Z. Fodor and A. Hebecker, Gauge invariant treatment of the electroweak phase transition, Phys. Lett. B 331 (1994) 131 [hep-ph/9403391] [INSPIRE].

[38] N.K. Nielsen, On the gauge dependence of spontaneous symmetry breaking in gauge theories, Nucl. Phys. B 101 (1975) 173 [INSPIRE].

[39] R. Fukuda and T. Kugo, Gauge invariance in the effective action and potential, Phys. Rev. D 13 (1976) 3469 [INSPIRE].

[40] I.J.R. Aitchison and C.M. Fraser, Gauge invariance and the effective potential, Annals Phys. 156 (1984) 1 [INSPIRE].

[41] D. Binosi, J. Papavassiliou and A. Pilaftsis, Displacement operator formalism for renormalization and gauge dependence to all orders, Phys. Rev. D 71 (2005) 085007 [hep-ph/0501259] [INSPIRE].

[42] D. Metaxas and E.J. Weinberg, Gauge independence of the bubble nucleation rate in theories with radiative symmetry breaking, Phys. Rev. D 53 (1996) 836 [hep-ph/9507381] [INSPIRE].

[43] M. Garny and T. Konstandin, On the gauge dependence of vacuum transitions at finite temperature, JHEP 07 (2012) 189 [arXiv:1205.3392] [INSPIRE].

[44] S.P. Martin, Taming the Goldstone contributions to the effective potential, Phys. Rev. D 90 (2014) 016013 [arXiv: 1406.2355] [INSPIRE].

[45] J. Elias-Miro, J.R. Espinosa and T. Konstandin, Taming infrared divergences in the effective potential, JHEP 08 (2014) 034 [arXiv: 1406.2652] [INSPIRE].

[46] J.M. Frere and P. Nicoletopoulos, Gauge invariant content of the effective potential, Phys. Rev. D 11 (1975) 2332 [INSPIRE].

[47] M. Sher, The renormalization group and inflationary potentials, Phys. Lett. B 135 (1984) 52 [INSPIRE].

[48] A.A. Starobinsky and J. Yokoyama, Equilibrium state of a selfinteracting scalar field in the de Sitter background, Phys. Rev. D 50 (1994) 6357 [astro-ph/9407016] [INSPIRE].

[49] G. Dvali and S. Kachru, New old inflation, hep-th/0309095 [InSPIRE]. 
[50] L. Pilo, A. Riotto and A. Zaffaroni, Old inflation in string theory, JHEP 07 (2004) 052 [hep-th/0401004] [INSPIRE].

[51] L. Pilo, A. Riotto and A. Zaffaroni, On the amount of gravitational waves from inflation, Phys. Rev. Lett. 92 (2004) 201303 [astro-ph/0401302] [INSPIRE].

[52] B. Freivogel, G.T. Horowitz and S. Shenker, Colliding with a crunching bubble, JHEP 05 (2007) 090 [hep-th/0703146] [inSPIRE].

[53] W. Israel, Singular hypersurfaces and thin shells in general relativity, Nuovo Cim. B 44S10 (1966) 1 [Erratum ibid. B 48 (1967) 463] [Nuovo Cim B 44 (1966) 1] [INSPIRE].

[54] S. Bartrum, A. Berera and J.G. Rosa, Fluctuation-dissipation dynamics of cosmological scalar fields, Phys. Rev. D 91 (2015) 083540 [arXiv:1412.5489] [InSPIRE].

[55] D.G. Figueroa, J. García-Bellido and F. Torrenti, The decay of the standard model Higgs after inflation, arXiv: 1504.04600 [INSPIRE].

[56] G.F. Giudice, A. Notari, M. Raidal, A. Riotto and A. Strumia, Towards a complete theory of thermal leptogenesis in the SM and MSSM, Nucl. Phys. B 685 (2004) 89 [hep-ph/0310123] [INSPIRE].

[57] C.P. Burgess, H.M. Lee and M. Trott, Power-counting and the validity of the classical approximation during inflation, JHEP 09 (2009) 103 [arXiv:0902.4465] [INSPIRE].

[58] J.L.F. Barbon and J.R. Espinosa, On the naturalness of Higgs inflation, Phys. Rev. D 79 (2009) 081302 [arXiv:0903.0355] [INSPIRE].

[59] V. Branchina and E. Messina, Stability, Higgs boson mass and new physics, Phys. Rev. Lett. 111 (2013) 241801 [arXiv:1307.5193] [INSPIRE].

[60] V. Branchina, E. Messina and M. Sher, Lifetime of the electroweak vacuum and sensitivity to Planck scale physics, Phys. Rev. D 91 (2015) 013003 [arXiv:1408.5302] [INSPIRE].

[61] F. Loebbert and J. Plefka, Quantum gravitational contributions to the standard model effective potential and vacuum stability, arXiv:1502.03093 [INSPIRE].

[62] P. Burda, R. Gregory and I. Moss, Gravity and the stability of the Higgs vacuum, Phys. Rev. Lett. 115 (2015) 071303 [arXiv:1501.04937] [INSPIRE].

[63] P. Burda, R. Gregory and I. Moss, Vacuum metastability with black holes, JHEP 08 (2015) 114 [arXiv: 1503.07331] [INSPIRE].

[64] E. Witten, Quantum gravity in de Sitter space, hep-th/0106109 [INSPIRE].

[65] N. Goheer, M. Kleban and L. Susskind, The trouble with de Sitter space, JHEP 07 (2003) 056 [hep-th/0212209] [INSPIRE].

[66] N. Arkani-Hamed, S. Dubovsky, L. Senatore and G. Villadoro, (No) eternal inflation and precision Higgs physics, JHEP 03 (2008) 075 [arXiv:0801.2399] [INSPIRE].

[67] D.N. Page, Is our universe decaying at an astronomical rate?, Phys. Lett. B 669 (2008) 197 [hep-th/0612137] [INSPIRE].

[68] S. Dubovsky, L. Senatore and G. Villadoro, The volume of the universe after inflation and de Sitter entropy, JHEP 04 (2009) 118 [arXiv:0812.2246] [INSPIRE].

[69] S. Dubovsky, L. Senatore and G. Villadoro, Universality of the volume bound in slow-roll eternal inflation, JHEP 05 (2012) 035 [arXiv:1111.1725] [INSPIRE]. 
[70] M. Lewandowski and A. Perko, Leading slow roll corrections to the volume of the universe and the entropy bound, JHEP 12 (2014) 060 [arXiv:1309.6705] [INSPIRE].

[71] N. Arkani-Hamed, S. Dubovsky, A. Nicolis, E. Trincherini and G. Villadoro, A measure of de Sitter entropy and eternal inflation, JHEP 05 (2007) 055 [arXiv:0704.1814] [INSPIRE].

[72] A.H. Guth and E.J. Weinberg, Could the universe have recovered from a slow first order phase transition?, Nucl. Phys. B 212 (1983) 321 [INSPIRE].

[73] M. Kleban, T.S. Levi and K. Sigurdson, Observing the multiverse with cosmic wakes, Phys. Rev. D 87 (2013) 041301 [arXiv: 1109.3473] [InSPIRE].

[74] S. Osborne, L. Senatore and K. Smith, Collisions with other universes: the optimal analysis of the WMAP data, arXiv:1305.1964 [INSPIRE].

[75] S. Osborne, L. Senatore and K. Smith, Optimal analysis of azimuthal features in the CMB, JCAP 10 (2013) 001 [arXiv:1305.1970] [INSPIRE].

[76] S.K. Blau, E.I. Guendelman and A.H. Guth, The dynamics of false vacuum bubbles, Phys. Rev. D 35 (1987) 1747 [INSPIRE].

[77] S. Chimento and D. Klemm, Multicentered black holes with a negative cosmological constant, Phys. Rev. D 89 (2014) 024037 [arXiv:1311.6937] [INSPIRE]. 